BULLETIN (New Series) OF THE

AMERICAN MATHEMATICAL SOCIETY

Volume 36, Number 2, Pages 135-249

S 0273-0979(99)00776-4

Article electronically published on February 19, 1999

\title{
ANALYTIC AND GEOMETRIC BACKGROUND OF RECURRENCE AND NON-EXPLOSION OF THE BROWNIAN MOTION ON RIEMANNIAN MANIFOLDS
}

\author{
ALEXANDER GRIGOR'YAN
}

\begin{abstract}
We provide an overview of such properties of the Brownian motion on complete non-compact Riemannian manifolds as recurrence and nonexplosion. It is shown that both properties have various analytic characterizations, in terms of the heat kernel, Green function, Liouville properties, etc. On the other hand, we consider a number of geometric conditions such as the volume growth, isoperimetric inequalities, curvature bounds, etc., which are related to recurrence and non-explosion.
\end{abstract}

\section{Contents}

1. Introduction 136

Acknowledgments $\quad 141$

Notation $\quad 141$

2. Heat semigroup on Riemannian manifolds $\quad 142$

2.1. Laplace operator of the Riemannian metric 142

2.2. Heat kernel and Brownian motion on manifolds 143

2.3. Manifolds with boundary 145

3. Model manifolds $\quad 145$

3.1. Polar coordinates 145

3.2. Spherically symmetric manifolds 146

$\begin{array}{ll}\text { 4. Some potential theory } & 149\end{array}$

4.1. Harmonic functions 149

4.2. Green function $\quad 150$

4.3. Capacity 152

4.4. Massive sets $\quad 155$

4.5. Hitting probabilities 159

4.6. Exterior of a compact 161

5. Equivalent definitions of recurrence 164

6. Equivalent definitions of stochastic completeness 170

7. Parabolicity and volume growth 174

7.1. Upper bounds of capacity 174

$\begin{array}{ll}\text { 7.2. Sufficient conditions for parabolicity } & 177\end{array}$

8. Transience and isoperimetric inequalities 181

9. Non-explosion and volume growth 184

Received by the editors October 1, 1997, and, in revised form, September 2, 1998.

1991 Mathematics Subject Classification. Primary 58G32, 58G11.

Research supported by the EPSRC Fellowship B/94/AF/1782 (United Kingdom). 
10. Transience and $\lambda_{1} \quad 191$

10.1. The first eigenvalue 191

10.2. Faber-Krahn inequality and transience 192

10.3. Heat kernel's upper bound 195

11. Transience and volume growth 197

11.1. Relative Faber-Krahn inequality 197

11.2. Covering manifolds 199

12. Transience on manifolds with a pole 200

13. Liouville properties 203

13.1. $L^{p_{-}}$-sub(super)harmonic functions 204

13.2. Liouville property for Schrödinger equation 205

13.3. Bounded harmonic functions 208

13.4. Minimal surfaces 209

13.5. Liouville property on Riemannian products 209

14. Harmonic functions on manifolds with ends 212

14.1. Parabolic subsets and ends 212

14.2. Spaces of harmonic functions on manifolds with ends 213

14.3. Manifolds with regular ends 214

14.4. Non-parabolicity of regular ends 216

15. Curvature and comparison theorems 220

15.1. Mean curvature 220

15.2. Sectional and Ricci curvature 223

15.3. Parabolicity for two dimensional manifolds 227

16. Heat kernel's lower bounds and recurrence of $\alpha$-process 232

17. Escape rate as a measure of transience 234

18. Problem section 236

References $\quad 242$

\section{Introduction}

The irregular movement of microscopic particles suspended in a liquid has been known since the beginning of the nineteenth century. In 1828, English botanist Robert Brown published his observations of ceaseless erratic motion of pollen grains in water. He emphasized the universal character of the phenomenon in contrast to the previous belief which attributed it to vital nature. That was the discovery of the physical phenomenon which was later named the Brownian motion.

It was not until 1905 when the effect was explained by Albert Einstein [56] as the result of the irregular collisions that the fine particles in suspension experience from molecules. Einstein realized the stochastic nature of the Brownian motion and proved that the probability distribution of the displacement of a Brownian particle satisfied a diffusion equation. Moreover, he computed the diffusion coefficient $D$ and made a prediction that the mean displacement of the particle during a time $t$ was $\sqrt{2 D t}$. The latter was confirmed experimentally by Jean Perrin in 1908 which later brought him a Nobel prize. That work was a strong argument in favor of the molecular-kinetic theory of heat and even the atomic structure of matter.

The simplest mathematical model of the Brownian motion is a random walk on a lattice. Suppose that a particle moves on the nodes of $\mathbb{Z}^{d}$ as follows. At each 


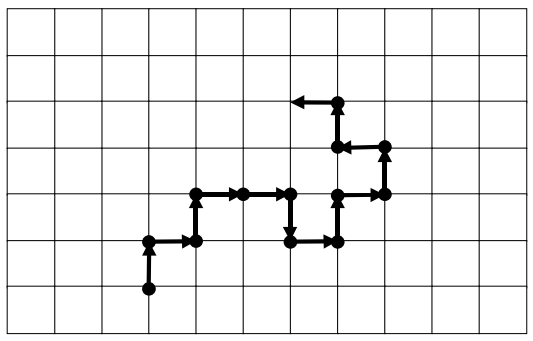

Figure 1. The random walk on $\mathbb{Z}^{2}$

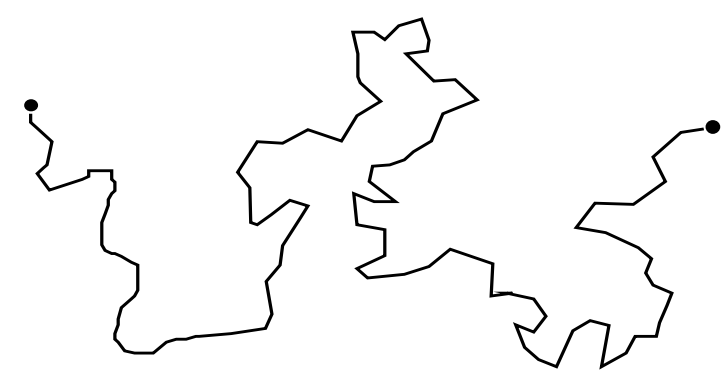

Figure 2. The Brownian motion on $\mathbb{R}^{2}$

step, it chooses randomly one of the $2 d$ neighboring nodes, with equal probability $(2 d)^{-1}$, and jumps to that node (see Figure 1).

A natural question is what happens with the particle as the number of steps $n \rightarrow \infty$ ? On average, the displacement of the particle is of the order $\sqrt{n}$ as in Einstein's model, but this does not say much about the trajectory of the particle. Since the rule of the movement of the particle is homogeneous and isotropic, one may expect that, in a long run, the number of moves in all $2 d$ directions should be approximately the same, and the particle should be regularly returning to a neighborhood of the origin. However, this is wrong! G.Pólya [159] discovered in 1921 that a long term behavior of the random walk depends on the dimension $d$. If $d \leq 2$ then the particle does visit every node (including the origin) infinitely many times, with probability 1 . However, if $d>2$ then the particle visits every node only finitely many times, also with probability 1 .

In other words, an observer of the two-dimensional random walk could see the particle in his/her range at arbitrarily large moments of time, whereas in the threedimensional space, the particle will escape from any bounded region after some time forever. The first type of behaviour of the particle is referred to as recurrence whereas the second one is called transience.

The same phenomenon takes place for a continuous time Brownian motion in $\mathbb{R}^{d}$ which can be obtained from the random walk on $\mathbb{Z}^{d}$ as a limit by a proper refinement of the lattice and the time step. A rigorous construction of a continuous model of the Brownian motion was done by Norbert Wiener [189]. This process is called the Wiener process and is recognized now as the standard model for the Brownian motion. Henceforth, we will use the term "the Brownian motion" as a synonym for the Wiener process (see Figure 2). 
One of the goals of this paper is to study the geometric background of the property of the Brownian motion to be recurrent or transient. In other words, what geometric properties of the state space causes the trajectory of the stochastic process to return to any region at arbitrarily large times or to leave any bounded region forever?

The answer to this question depends on the family of the state spaces in question. In the category of the Euclidean spaces (or lattices) one may answer that it is the dimension which makes the difference. However, this becomes totally wrong in the category of Riemannian manifolds. A Riemannian manifold is an abstract version of a smooth curved surface. This is rather a point of view that takes into account only intrinsic properties of the surface, which do not depend on the embedding space.

Riemannian manifolds provide rich enough family of geometries. For example, all classical model geometries - the spherical, euclidean and hyperbolic geometries - are particular cases of Riemannian geometries. It turns out that the continuous Brownian motion can be constructed on any Riemannian manifold (imagine a Brownian particle moving on a curved surface). The Brownian motion on a Riemannian manifold is called recurrent if it visits any open set at arbitrarily large moments of time with probability 1 , and transient otherwise.

We shall see that recurrence is related to various geometric properties of the underlying Riemannian manifold such as the volume growth, isoperimetric inequalities, curvature etc. On the other hand, recurrence happens to be equivalent to certain potential-theoretic properties of the Laplace operator on the manifold. For example, the recurrence of the Brownian motion in $\mathbb{R}^{2}$ is linked to the fact that the fundamental solution $\log |x|$ of the Laplace operator in $\mathbb{R}^{2}$ is signed as opposed to the positivity of the fundamental solution $|x|^{2-d}$ in $\mathbb{R}^{d}$, for $d>2$.

The question of recurrence of the Brownian motion on Riemann surfaces goes back to the uniformization theorem of F.Klein, P.Koebe and H.Poincaré. This celebrated theorem says, in particular, that any simply connected Riemann surface is conformally equivalent to one of the following canonical surfaces:

1. the sphere (surface of elliptic type)

2. the Euclidean plane (surface of parabolic type)

3. the hyperbolic plane (surface of hyperbolic type).

The problem of deciding what is the type of a given Riemann surface is known as the type problem. It is easy to distinguish between the elliptic type and the others - the former is compact whereas the latter are not. A more interesting question is how to distinguish the parabolic and hyperbolic types by using intrinsic geometric properties of Riemann surfaces. Amazingly, the parabolicity is exactly equivalent to the recurrence of the Brownian motion on the Riemann surface in question.

The understanding of parabolicity of Riemann surfaces from the potential -theoretic point of view is largely due to L.Ahlfors [1] - [3], P.J.Myrberg [144], R.Nevanlinna [149], [150] and H.Royden [163]. J.Deny [47], G.Hunt [98] and S.Kakutani [105] contributed to the potential-theoretic background of recurrence. The study of recurrence in connection with the geometry of Riemannian manifolds was boosted by the works of S.Y.Cheng and S.-T.Yau [28] and N.Varopoulos [183], [184].

Another property of the Brownian motion to be considered in this paper is stochastic completeness. This is a property of a stochastic process to have infinite 
lifetime. In other words, a process is stochastically complete if the total probability of the particle being found in the state space is constantly equal to 1 . This is also referred to as a conservation property (of probability) or non-explosion. Despite the fact that the very term "a probability measure" means a measure with the total mass 1, there are simple examples of stochastically incomplete processes. Consider a Brownian motion in a bounded region $\Omega \subset \mathbb{R}^{d}$ with an absorbing boundary. After hitting the boundary $\partial \Omega$, the particle dies, and this happens with a positive probability. Therefore, at any positive time, the total probability of the particle being found in $\Omega$ is smaller than 1 .

The Brownian motion in $\mathbb{R}^{d}$ (and the standard random walk in $\mathbb{Z}^{d}$ ) is stochastically complete. One might wonder whether stochastic incompleteness has to do only with the presence of some killing conditions. It turns out that even without any killing, there may exist a geometric reason for stochastic incompleteness. R.Azencott [6] showed that the Brownian motion on a Riemannian manifold $M$ may be stochastically incomplete even if $M$ is geodesically complete. Note that a bounded region $\Omega \subset \mathbb{R}^{d}$ is not geodesically complete when considered as a manifold.

In the example of R.Azencott, the manifold $M$ has a negative sectional curvature which grows fast enough to $-\infty$ with the distance from an origin. The stochastic incompleteness of the Brownian motion on $M$ occurs because negative curvature on a manifold plays the role of a drift to infinity, and a very high negative curvature may produce an extremely fast drift which sweeps a Brownian particle to infinity in a finite time.

An interesting question is to understand precisely what geometric properties of $M$ ensure stochastic completeness or incompleteness of the Brownian motion. The crucial contributions here are due to R.Azencott [6], M.Gaffney [66], R.Khas'minskii [111] and S.-T.Yau [194].

One may wonder what recurrence and the conservation property have in common. Both transience and explosion (=stochastic incompleteness) reflect the tendency of the Brownian motion to escape to infinity. While transience says that the Brownian motion escapes to infinity, explosion means that it does it in a finite time. There is a full range of various escape rates between transience and explosion. No wonder that many conditions of recurrence of Brownian motion on manifolds have their counterparts for stochastic completeness.

The purpose of this paper is to expose the relationship between recurrence and the conservation property on the one hand, and many other geometric and potentialanalytic properties on the other hand. For example, recurrence can be characterized in terms of a fundamental solution to the Laplace equation, superharmonic functions, capacities, the heat kernel, the Liouville property for certain Schrödinger equation, etc. The stochastic completeness can also be characterized in various terms including the uniqueness in the Cauchy problem for the heat equation in the class of bounded solutions. Given that much, these two properties of the Brownian motion appear to be of fundamental importance for analysis on manifolds and for adjacent areas.

This paper splits into two parts. The first part consists of sections $2-9$ and is rather elementary. It focuses on the one hand on Theorems 5.1 and 6.2, which contain various characterizations of transience and explosion partly already mentioned above, and on the other hand on Theorems 7.3 and 9.1, which give the following sufficient conditions for recurrence and non-explosion in terms of the volume growth. 
Let $V(r)$ denote the Riemannian volume of a geodesic ball of radius $r$ with a fixed center. Then the Brownian motion on a geodesically complete manifold $M$ is recurrent provided

$$
\int^{\infty} \frac{r d r}{V(r)}=\infty
$$

For example, this condition is satisfied if $V(r) \leq C r^{2}$. In particular, this explains why the Brownian motion on $\mathbb{R}^{2}$ is recurrent - there is just not enough volume in the two-dimensional Euclidean space!

Furthermore, the Brownian motion on $M$ is stochastically complete provided

$$
\int^{\infty} \frac{r d r}{\log V(r)}=\infty
$$

For example, this condition is satisfied if $V(r) \leq \exp \left(C r^{2}\right)$. Clearly, all Euclidean spaces fit into this volume growth. This explains why we have to move to manifolds in order to observe stochastic incompleteness - $\mathbb{R}^{d}$ is too small for that!

Full proofs of the key results of the first part are provided, which should be accessible for graduate students with adequate background. Let us emphasize that we normally assume known (and, thus, do not provide proof for) all facts which depend only on the local structure of the manifold. For example, we freely use such properties of solutions to the elliptic equations as $C^{\infty}$-regularity, convergence principles, maximum principle, solvability of the Dirichlet problem etc. On the contrary, we concentrate on the properties related to the structure of the manifold "in the large".

The second part is devoted to the relations of recurrence and non-explosion to other questions such as Liouville properties, heat kernel bounds, eigenvalues of the Laplace operator, curvature, escape rate of the Brownian motion, etc. This part is more advanced and sketchy, and some results may appear to be new even to the experts. However, we do not aim to include the most recent and general results, and we have opted for those which exhibit interesting phenomena without technical complications.

The subject of this paper lies on the borderline between different fields of mathematics such as Riemannian Geometry, Stochastic Analysis, Partial Differential Equations and Potential Theory. We refer the reader to the following textbooks for the necessary background:

- Riemannian Geometry: [22], [21], [112], [158], [169].

- Analysis and PDE: [43], [68], [116], [134], [188].

- Probability: [55], [60], [103], [133], [136], [172].

- Potential Theory: [16], [30], [32], [51], [64], [179].

The following monographs and survey articles provide additional valuable information about elliptic and parabolic differential equations on manifolds: [5], [69], [92], [130], [140], [113], [161], [162], [167], [170], [180].

There is also a vast literature devoted to recurrence criteria for random walks on graphs and lattices. We do not touch this question here and refer an interested reader to the books [53] and [191] as well as to the surveys [67], [90], [166], [190]. 


\section{ACKNOWLEDGMENTS}

The author is obliged to Mike Cranston, Thierry Coulhon, Minoru Murata and Laurent Saloff-Coste for numerous suggestions for improving the original manuscript. He is also grateful to David Hoffman and Robert Osserman for their interest in this work and for encouragement.

\section{NotATION}

The following list of notation is provided for convenience of reading. Most of them are also explained in the main body of the paper.

$M$ - a smooth connected Riemannian manifold. In many cases, $M$ is geodesically complete and non-compact.

$d \geq 2$ - the (topological) dimension of $M$.

$\operatorname{dist}(x, y)$ - the geodesic distance on $M$ between the points $x, y \in M$.

$\mu$ - the Riemannian volume on $M$.

$\mu^{\prime}$ - the Riemannian measure of the co-dimension 1 on hypersurfaces in $M$.

$|A|:=\mu(A)$ or $\mu^{\prime}(A)$ depending on the context (for example, if $A$ is an open subset of $M$, then $|A|=\mu(A)$; whereas if $A$ is a boundary of an open subset, then $\left.|A|=\mu^{\prime}(A)\right)$.

$B(x, r)$ - the geodesic ball of radius $r$ centered at the point $x \in M$.

$V(x, r):=\mu(B(x, r))$ - the volume growth function.

$\Delta$ - the Laplace -Beltrami operator on $M$.

$\lambda_{1}(\Omega)$ - the first eigenvalue of the Dirichlet problem for $\Delta$ in $\Omega$ (where $\Omega$ is a region in $M)$.

$p(t, x, y)$ - the heat kernel associated with the operator $\frac{1}{2} \Delta$.

$p_{\Omega}(t, x, y)$ - the heat kernel in the region $\Omega$ with the Dirichlet boundary condition on $\partial \Omega$.

$P_{t}$ - the heat semigroup with the kernel $p(t, x, y)$.

$P_{t}^{\Omega}$ - the heat semigroup with the kernel $p_{\Omega}(t, x, y)$.

$G(x, y)$ - the Green kernel of $-\Delta$ on $M$.

$G_{\Omega}(x, y)$ - the Green kernel of $-\Delta$ in the region $\Omega$ with the Dirichlet boundary condition.

$X_{t}$ - the Brownian motion on $M$ generated by $\frac{1}{2} \Delta$.

$\mathbb{P}_{x}, \mathbb{E}_{x}$ - measure and expectation, respectively, on the space of paths of the Brownian motion started at $x \in M$.

$b_{\Omega}, s_{\Omega}$ - the subharmonic and superharmonic potentials of an open set $\Omega$ - see Section 4.4.

$e_{F}, h_{F}$ - the hitting probabilities - see Section 4.5.

$C_{0}^{\infty}(\Omega)$ - the set of smooth real-valued functions on $\Omega$ with compact support in $\Omega$.

$\mathcal{L}(K, \Omega)$ - the set of locally Lipschitz functions $\phi$ on $M$, compactly supported in $\Omega$ and such that $0 \leq \phi \leq 1$ and $\left.\phi\right|_{K}=1$.

$\left\{\mathcal{E}_{k}\right\}$ - an exhaustion sequence on $M$ - see Section 4.2.

$x \rightarrow \infty$ - a sequence $\left\{x_{k}\right\}$ on $M$ which leaves any compact set after some $k$. If $M$ is geodesically complete, then this is equivalent to $\operatorname{dist}(x, o) \rightarrow \infty$ where $o$ is a reference point.

flux $f$ - the flux of the function $f$ through a smooth oriented hypersurface $\Gamma$, that is, $\int_{\Gamma} \frac{\partial f}{\partial \nu} d \mu^{\prime}$ where $\nu$ is a unit normal vector field on $\Gamma$ associated with the 
orientation of $\Gamma$. If $\Gamma$ is a boundary of an open set $\Omega$, then $\nu$ points outwards from $\Omega$.

$\mathbb{S}^{d}$ - the $d$-dimensional unit sphere in $\mathbb{R}^{d+1}$.

$\mathbb{H}^{d}$ - the $d$-dimensional hyperbolic space.

$\omega_{d}$ - the boundary area of the unit sphere in $\mathbb{R}^{d}$.

const - a positive constant which may be different at different occurrences.

\section{HEAT SEMIGROUP ON RIEMANNIAN MANIFOLDS}

The simplest way to construct Brownian motion on a Riemannian manifold $M$ is to construct first the heat kernel which will also serve as the density of the transition probability. The heat kernel will be denoted by $p(t, x, y)$ where $t>0$ is a time, $x, y$ are points on $M$. Thus, the probability that the Brownian motion starting at the point $x$ lies in a measurable set $\Omega \subset M$ at the time $t$ is given by

$$
\int_{\Omega} p(t, x, y) d \mu(y)
$$

In $\mathbb{R}^{d}$, the heat kernel is given by the classical formula

$$
p(t, x, y)=\frac{1}{(2 \pi t)^{d / 2}} \exp \left(-\frac{|x-y|^{2}}{2 t}\right) .
$$

It is known to satisfy the heat equation

$$
\frac{\partial p}{\partial t}-\frac{1}{2} \Delta p=0
$$

in the variables $(t, x)$ (the point $y$ is considered as fixed) and the initial data

$$
p(t, \cdot, y) \underset{t \rightarrow 0+}{\longrightarrow} \delta_{y}
$$

where $\delta_{y}$ is the delta function of Dirac.

The properties (2.1) and (2.2) can be used to define the heat kernel on an arbitrary Riemannian manifold $M$, which is done below.

2.1. Laplace operator of the Riemannian metric. Let $g_{i j}$ be the Riemannian metric tensor on $M$. This means that, in any coordinate chart $\left(x^{1}, x^{2}, \ldots, x^{d}\right)$ on $M$, the length element can be computed by

$$
d s^{2}=g_{i j} d x^{i} d x^{j}
$$

where we assume the summation on the repeated indices. Denote by $g^{i j}$ the elements of the inverse matrix $\left\|g_{i j}\right\|^{-1}$ and let $g:=\operatorname{det}\left\|g_{i j}\right\|$. Then the Laplace operator $\Delta$ associated with the metric $g_{i j}$ is defined by

$$
\Delta=\frac{1}{\sqrt{g}} \frac{\partial}{\partial x^{i}}\left(\sqrt{g} g^{i j} \frac{\partial}{\partial x^{j}}\right) .
$$

This is a second order elliptic operator on $M$. It is possible to show that (2.3) defines the same operator in different charts.

Sometimes it is useful to represent the Laplacian in the form

$$
\Delta=\operatorname{div} \nabla
$$

where the gradient $\nabla$ acts on a function $f$ by

$$
(\nabla f)^{i}=g^{i j} \frac{\partial f}{\partial x^{j}}
$$


and the divergence div acts on a vector field $F=F^{i} \frac{\partial}{\partial x^{i}}$ by

$$
\operatorname{div} F=\frac{1}{\sqrt{g}} \frac{\partial}{\partial x^{i}}\left(\sqrt{g} F^{i}\right) .
$$

Green's formula, which follows easily from Stokes's theorem, says that, for any precompact region $U$ and for any functions $u, v \in C_{0}^{2}(U)$,

$$
\int_{U} v \Delta u d \mu=-\int_{U} \nabla v \nabla u d \mu
$$

where $\nabla v \nabla u$ is the inner (Riemannian) product of the vectors

$$
\nabla v \nabla u=g_{i j}(\nabla u)^{i}(\nabla v)^{j}=g^{i j} \frac{\partial u}{\partial x^{i}} \frac{\partial v}{\partial x^{j}},
$$

and $d \mu$ is the Riemannian volume element, which is defined by

$$
d \mu=\sqrt{g} d x^{1} d x^{2} \ldots d x^{d} .
$$

If the boundary $\partial U$ is smooth enough (say, $C^{1}$ ) and $u, v \in C^{2}(U) \cap C^{1}(\bar{U})$, then we have the following version of (2.4) with a boundary term

$$
\int_{U} v \Delta u d \mu=\int_{\partial U} v \frac{\partial u}{\partial \nu} d \mu^{\prime}-\int_{U} \nabla v \nabla u d \mu,
$$

where $d \mu^{\prime}$ in the middle integral is the Riemannian volume element on the submanifold $\partial U$, and $\nu$ is the outward unit normal vector field on $\partial U$.

2.2. Heat kernel and Brownian motion on manifolds. Any function on $(0, \infty)$ $\times M \times M$ satisfying (2.1) and (2.2) is called a fundamental solution of the heat equation (2.1) on $M$. The heat kernel is the smallest positive fundamental solution of the heat equation on $M$. It was proved by J.Dodziuk [48] that the heat kernel always exists (regardless of geodesic completeness) and is smooth in $(t, x, y)$. Moreover, the heat kernel possesses the following properties.

1. Symmetry in $x, y$, that is $p(t, x, y)=p(t, y, x)$.

2. The semigroup identity: for any $s \in(0, t)$

$$
p(t, x, y)=\int_{M} p(s, x, z) p(t-s, z, y) d \mu(z) .
$$

3. For all $t>0$ and $x \in M$,

$$
\int_{M} p(t, x, y) d \mu(y) \leq 1
$$

As soon as one has (2.7) and (2.8), a (sub)Markov process $X_{t}$ on $M$ can be constructed with the transition density $p$ by using the standard probabilistic tools (see [30], [55]). The process $X_{t}$ turns out to be a diffusion and is referred to as the Brownian motion or the Wiener process on $M$. The corresponding measure in the space of paths emanating from a point $x$ will be denoted by $\mathbb{P}_{x}$.

Given an open set $\Omega \subset M$, one can treat $\Omega$ as a manifold itself. Let us denote by $p_{\Omega}$ the heat kernel of $\Omega$. Minimality of the heat kernel implies that $p_{\Omega}$ vanishes on the boundary $\partial \Omega$, at least if $\partial \Omega$ is smooth. This implies, in turn, that $p_{\Omega}$ increases on enlarging of $\Omega$. 
The way the global heat kernel $p$ is constructed in [48] is the following: one first defines $p_{\Omega}$ for precompact sets $\Omega$ (which can be done by using the eigenfunction expansion) and then lets

$$
p:=\lim _{k \rightarrow \infty} p_{\Omega_{k}}
$$

where $\left\{\Omega_{k}\right\}$ is an increasing sequence of precompact open sets with smooth boundaries, which exhaust $M$.

Due to (2.7) and (2.8), the heat kernel $p(x, y, t)$ can be considered as a kernel of the submarkovian operator semigroup $P_{t}$ which acts on functions on $M$ by

$$
P_{t} f:=\int_{M} p(\cdot, y, t) f(y) d \mu(y) .
$$

The semigroup corresponding to $p_{\Omega}$ will be denoted by $P_{t}^{\Omega}$. If $f$ is a continuous bounded function on $M$, then the function $u(x, t):=P_{t} f(x)$ solves the Cauchy problem in $M \times(0, \infty)$ :

$$
\left\{\begin{array}{l}
\frac{\partial u}{\partial t}=\frac{1}{2} \Delta u \\
u(\cdot, 0)=f
\end{array}\right.
$$

Moreover, if $f \geq 0$ then $P_{t} f$ is the smallest non-negative solution to this problem. See [171] for detailed properties of the heat semigroup on manifolds.

Let us briefly mention another way of constructing the heat kernel on Riemannian manifolds which goes back to Gaffney [65] and which was implemented in full generality by Cheeger and Yau [27]. The idea is to consider $\Delta$ as an unbounded operator in $L^{2}(M, \mu)$. It is possible to prove that the operator $\Delta$ with the domain $C_{0}^{\infty}(M)$ is essentially self-adjoint and non-positive. Therefore, by using the spectral theory, one can construct the one-parameter operator semigroup $e^{\frac{1}{2} t \Delta}$ acting in $L^{2}(M, \mu)$. Next, one proves that this semigroup possesses a smooth kernel which is the heat kernel (see also [162], [169, p.94]). The equivalence of these two approaches was proved in [48].

Other methods of constructing the Brownian motion on manifolds (or even on more general underlying spaces) can be found in [6], [57], [63], [102], [133], [136].

Now we can precisely define the recurrence and conservation properties.

Definition 2.1. Brownian motion $X_{t}$ on a manifold $M$ is recurrent if, for any non-empty open set $\Omega$ and for any point $x \in M$,

$$
\mathbb{P}_{x}\left\{\text { there is a sequence } t_{k} \rightarrow \infty \text { such that } X_{t_{k}} \in \Omega\right\}=1 .
$$

Otherwise $X_{t}$ is transient.

Definition 2.2. Brownian motion $X_{t}$ is stochastically complete (=possesses the conservation property or the non-explosion property) if, for all $x \in M$ and $t>0$,

$$
\int_{M} p(t, x, y) d \mu(y)=1
$$

(in other words, $\mathbb{P}_{x}$ is a probability measure in the sense that its total mass is equal to 1$)$.

It is convenient to say that a manifold is stochastically complete (recurrent, transient) when the Brownian motion on it has this property. 
2.3. Manifolds with boundary. Sometimes it is useful to allow a manifold $M$ to possess a boundary $\partial M$. In this case, we assume that the heat kernels $p(t, x, y)$ and $p_{\Omega}(t, x, y)$ satisfy in addition the Neumann boundary condition on $\partial M$ and $\partial M \cap \Omega$ respectively. For the Brownian motion this means that $X_{t}$ reflects on $\partial M$.

Most results of this paper remain true for manifolds with boundary. However, by default we consider manifolds without boundary in order to avoid some technical complications.

\section{MOdel MANIFOLDS}

The purpose of this section is to introduce a class of model manifolds which are the manifolds with rotational symmetry.

3.1. Polar coordinates. Let us fix a point $o \in M$ and denote by $C u t(o)$ the cut locus of $o$. Away from $C u t^{*}(o):=\operatorname{Cut}(o) \cup\{o\}$, one can define the polar coordinates with the pole $o$ (see Figure 3). Namely, for any point $x \in M \backslash C u t^{*}(o)$ there corresponds a polar radius $\rho:=\operatorname{dist}(x, o)$ and a polar "angle" $\theta \in \mathbb{S}^{d-1}$ such that the shortest geodesics from $o$ to $x$ start at $o$ to the direction $\theta$ in $T_{o} M$. We can identify $T_{o} M$ with $\mathbb{R}^{d}$ so that $\theta$ can be regarded as a point on $\mathbb{S}^{d-1}$. In particular, $M \backslash C u t^{*}(o)$ is diffeomorphic to a star-like region on $\mathbb{R}_{+} \times \mathbb{S}^{d-1}$ (see [112] and [69] for proofs of the facts mentioned here).

The Riemannian metric in $M \backslash C u t^{*}(o)$ has in the polar coordinates the form

$$
d s^{2}=d \rho^{2}+A_{i j}(\rho, \theta) d \theta^{i} d \theta^{j},
$$

where $\left(\theta^{1}, \theta^{2}, \ldots, \theta^{d-1}\right)$ are coordinates on $\mathbb{S}^{d-1}$ and $\left\|A_{i j}(\rho, \theta)\right\|$ is a positive definite matrix. In fact, $A_{i j}(\rho, \cdot)$ is the Riemannian metric tensor on the geodesic sphere $S_{\rho}:=\partial B(o, \rho) \backslash \operatorname{Cut}(o)$. Denote $\mathrm{A}=\operatorname{det}\left\|A_{i j}\right\|$. Then we have, by (2.5), the area element on $S_{\rho}$

$$
\left.d \mu^{\prime}\right|_{S_{\rho}}=\sqrt{\mathrm{A}} d \theta^{1} d \theta^{2} \ldots d \theta^{d-1}
$$

In particular,

$$
\mu^{\prime}\left(S_{\rho}\right)=\int_{\mathbb{S}^{d-1}} \sqrt{\mathrm{A}} d \theta^{1} d \theta^{2} \ldots d \theta^{d-1}
$$

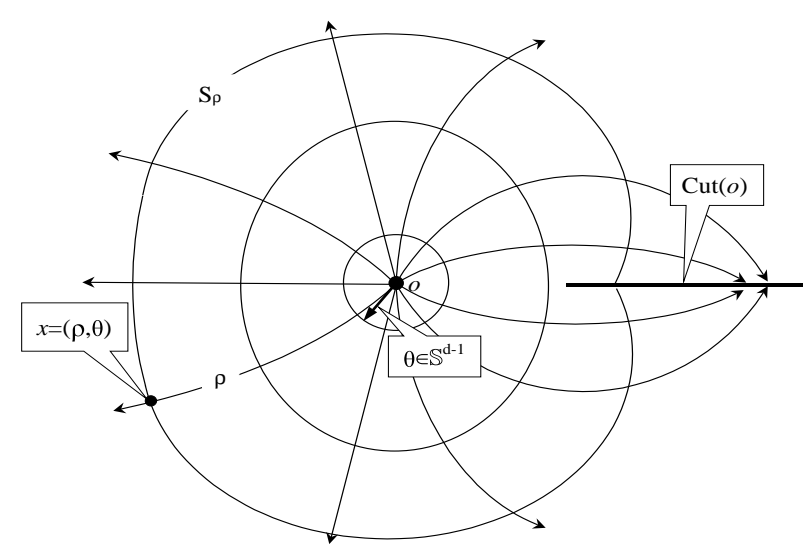

Figure 3. Polar coordinates in $M \backslash C u t^{*}(o)$ 


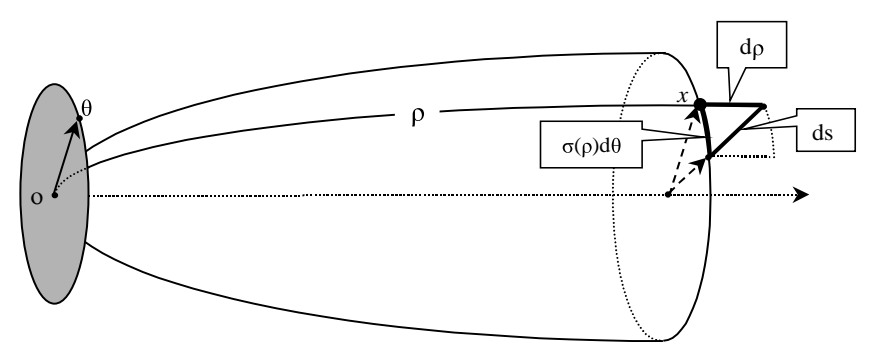

FiguRE 4. The metric on the surface of revolution $M_{\sigma}$

assuming that $\theta^{1}, \theta^{2}, \ldots, \theta^{d-1}$ are defined almost everywhere on $\mathbb{S}^{d-1}$.

It follows easily from (2.3) and (3.1) that the Laplace operator has in the polar coordinates the form

$$
\Delta=\frac{1}{\sqrt{\mathrm{A}}} \frac{\partial}{\partial \rho}\left(\sqrt{\mathrm{A}} \frac{\partial}{\partial \rho}\right)+\Delta_{S_{\rho}}=\frac{\partial^{2}}{\partial^{2} \rho}+(\log \sqrt{\mathrm{A}})^{\prime} \frac{\partial}{\partial \rho}+\Delta_{S_{\rho}}
$$

where $(\cdot)^{\prime}$ denotes $\partial / \partial \rho$ and $\Delta_{S_{\rho}}$ is the Laplace operator on the submanifold $S_{\rho}$.

We say that $M$ is a manifold with a pole if $M$ possesses a point $o$ with an empty cut locus $C u t(o)$. The point $o$ is called the pole of $M$, and the polar coordinates are defined on $M \backslash\{o\}$. If, in addition, $M$ is geodesically complete, then $M$ is diffeomorphic to $\mathbb{R}^{d}$.

3.2. Spherically symmetric manifolds. In the next sections, we will introduce methods for determining whether a given manifold is recurrent or stochastically complete. The simplest class of manifolds where these methods apply and give straightforward answers is the class of spherically symmetric manifolds.

A manifold $M$ with a pole $o$ is called a spherically symmetric manifold or a model if the Riemannian metric on $S_{\rho}$ (see (3.1)) is given by

$$
A_{i j}(\rho, \theta) d \theta^{i} d \theta^{j}=\sigma^{2}(\rho) d \theta^{2}
$$

where $d \theta^{2}$ is the standard Euclidean metric $\mathbb{S}^{d-1}$ and $\sigma(\rho)$ is a smooth positive function of $\rho$. In other words, the Riemannian metric on $S_{\rho}$ is obtained by scaling that of $\mathbb{S}^{d-1}$ by the factor $\sigma^{2}$.

Given a smooth positive function $\sigma(\rho)$ on $\left(0, R_{0}\right)$, the necessary and sufficient condition that such a manifold exists is

$$
\sigma(0)=0 \quad \text { and } \quad \sigma^{\prime}(0)=1 .
$$

The hypotheses (3.5) ensure that the metric on the cone $\left(0, R_{0}\right) \times \mathbb{S}^{d-1}$ defined by

$$
d s^{2}=d \rho^{2}+\sigma^{2}(\rho) d \theta^{2},
$$

can be smoothly extended to the origin $\rho=0$ (see [69]). We assume in the sequel that $\sigma$ satisfies $(3.5)$ and denote by $M_{\sigma}$ the cone $\left(0, R_{0}\right) \times \mathbb{S}^{d-1}$ with the added origin.

Clearly, the model manifold $M_{\sigma}$ is diffeomorphic to an open ball in $\mathbb{R}^{d}$ of radius $R_{0}$ (or the whole $\mathbb{R}^{d}$ if $R_{0}=\infty$ ). The metric on any geodesic sphere $\partial B(o, r)$ on $M_{\sigma}$ is obtained from that of $\mathbb{S}^{d-1}$ by scaling it by the factor $\sigma(r)$. In certain situations, $M_{\sigma}$ can be regarded as a surface of revolution in $\mathbb{R}^{d+1}$ (see Figure 4 ). 


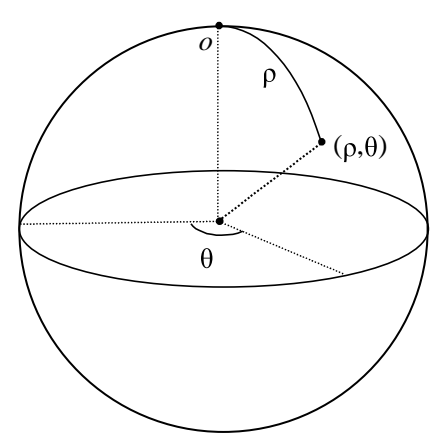

FiguRE 5 . The polar coordinates on $\mathbb{S}^{2}$

Since $\sqrt{\mathrm{A}}=\sigma^{d-1}$, we see from (3.3) that the boundary area $S(r)$ of the geodesic sphere $\partial B(o, r)$ is computed by

$$
S(r)=\omega_{d} \sigma^{d-1}(r),
$$

where $\omega_{d}$ is the area of the unit sphere in $\mathbb{R}^{d}$. The volume $V(r)$ of the ball $B(o, r)$ is given by

$$
V(r)=\int_{0}^{r} S(\xi) d \xi=\omega_{d} \int_{0}^{r} \sigma^{d-1}(\xi) d \xi
$$

The Laplace operator on $M_{\sigma}$ can be written down as follows (cf. (3.4))

$$
\Delta=\frac{\partial^{2}}{\partial^{2} \rho}+(d-1) \frac{\sigma^{\prime}}{\sigma} \frac{\partial}{\partial \rho}+\frac{1}{\sigma^{2}} \Delta_{\theta}
$$

or

$$
\Delta=\frac{\partial^{2}}{\partial^{2} \rho}+\frac{S^{\prime}}{S} \frac{\partial}{\partial \rho}+\frac{1}{\sigma^{2}} \Delta_{\theta}
$$

where $\Delta_{\theta}$ denotes the Laplace operator on the sphere $\mathbb{S}^{d-1}$. It is important that the operator $\Delta_{\theta}$ does not depend on the variable $\rho$.

The major examples of model manifolds are as follows.

Example 3.1. If $R_{0}=\infty$ and

$$
\sigma(r)=r,
$$

then $M_{\sigma}$ is isometric to $\mathbb{R}^{d}$. The boundary area is

$$
S(r)=\omega_{d} r^{d-1}
$$

and the Laplace operator is

$$
\Delta=\frac{\partial^{2}}{\partial^{2} \rho}+\frac{d-1}{\rho} \frac{\partial}{\partial \rho}+\frac{1}{\rho^{2}} \Delta_{\theta} .
$$

Example 3.2. Let us set

$$
\sigma(r)=\sin r
$$

Then $M_{\sigma}$ is the sphere $\mathbb{S}^{d}$ (assuming that $R_{0}$ takes the maximum possible value $\pi$ and that the endpoint with $\rho=\pi$ is added to $M_{\sigma}$ ). If $d=2$ then $r$ becomes the latitude measured from the pole, and $\theta$ is the longitude (see Figure 5 ). 
The boundary area is

$$
S(r)=\omega_{d} \sin ^{d-1} r .
$$

The Laplace operator on $\mathbb{S}^{d}$ acquires the form

$$
\Delta=\frac{\partial^{2}}{\partial^{2} \rho}+(d-1) \cot \rho \frac{\partial}{\partial \rho}+\frac{1}{\sin ^{2} \rho} \Delta_{\theta},
$$

where $\Delta_{\theta}$ is the Laplace operator on the sphere $\mathbb{S}^{d-1}$. This formula can be iterated in the dimension $d$ to produce a full expansion of the spherical Laplace operator in the angular coordinates.

Example 3.3. Let us set

$$
\sigma(r)=\sinh r .
$$

Then $M_{\sigma}$ is the hyperbolic space $\mathbb{H}^{d}$ - the complete simply connected $d$-dimensional manifold with constant sectional curvature -1 (assuming $R_{0}=\infty$ ). The boundary area on $\mathbb{H}^{d}$ is equal to

$$
S(r)=\omega_{d} \sinh ^{d-1} r
$$

and the Laplace operator is

$$
\Delta=\frac{\partial^{2}}{\partial^{2} \rho}+(d-1) \operatorname{coth} \rho \frac{\partial}{\partial \rho}+\frac{1}{\sinh ^{2} \rho} \Delta_{\theta} .
$$

It turns out that recurrence and stochastic completeness of $M_{\sigma}$ can easily be determined via the boundary area $S(r)$. The following tests were proved by many authors in various settings ([1], [139], [111], [99], [100], [75]).

Proposition 3.1. Let $M_{\sigma}$ be a model manifold with $R_{0}=\infty$ (so that $M_{\sigma}$ is geodesically complete and non-compact). Then $M_{\sigma}$ is recurrent if and only if

$$
\int^{\infty} \frac{d r}{S(r)}=\infty
$$

Proposition 3.2. Let $M_{\sigma}$ be a model manifold with $R_{0}=\infty$. Then $M$ is stochastically complete if and only if

$$
\int^{\infty} \frac{V(r)}{S(r)} d r=\infty
$$

The proofs will be given in Sections 5 and 6 respectively. ${ }^{1}$ The condition (3.8) holds for

$$
S(r) \leq \text { const } r, \quad r \rightarrow \infty
$$

and fails if

$$
S(r) \geq \operatorname{const} r^{1+\varepsilon}, \quad \varepsilon>0 .
$$

This explains why $\mathbb{R}^{d}$ is recurrent for $d \leq 2$ and transient if $d>2$. The hyperbolic space is transient because $S(r)$ on $\mathbb{H}^{d}$ grows exponentially fast.

\footnotetext{
${ }^{1}$ If one neglects the angular direction, then recurrence and non-explosion on a model manifold amount to the same properties for the one-dimensional diffusion on $(0, \infty)$ generated by the operator $\frac{d^{2}}{d^{2} \rho}+\frac{S^{\prime}}{S} \frac{d}{d \rho}$. The results of Feller [58] and Khas'minskii [111, pp.193-194] cover such diffusions and yield exactly the tests (3.8) and (3.9).
} 
The borderline for the stochastic completeness condition (3.9) is much higher: it holds, for example, if, for large $r$,

$$
S(r)=\exp \left(r^{2}\right)
$$

and fails if

$$
S(r)=\exp \left(r^{2+\varepsilon}\right), \quad \varepsilon>0
$$

The latter yields an example of a geodesically complete but stochastically incomplete manifold.

\section{Some Potential theory}

In this section, we will give an analytic characterization of certain hitting probabilities. Let $F$ be a closed subset of $M$. Denote by $e_{F}(x)$ the $\mathbb{P}_{x}$-probability that the process $X_{t}$ hits $F$ ever, that is, $X_{t} \in F$ for some $t \geq 0$. Obviously, $e_{F}(x)=1$ on $F$. It turns out that $e_{F}(x)$ is a harmonic function outside $F$ and superharmonic on $M$.

Another function $h_{F}(x)$ to be considered here is the $\mathbb{P}_{x}$-probability of the event that $X_{t}$ visits $F$ at arbitrarily large moments of time. This function turns out to be harmonic on all of $M$ (see Propositions 4.3 and 4.4 below). Both hitting probabilities play an important role in the part of this paper devoted to recurrence.

4.1. Harmonic functions. A function $u$ defined in a region of $M$ is harmonic if

$$
\Delta u=0 .
$$

The equation (4.1) is understood either in the sense of distribution or pointwise. In the latter case, the function $u$ is initially $C^{2}$ smooth. In both cases, $u$ will be actually $C^{\infty}$. Indeed, $u$ satisfies locally an elliptic equation of the second order with smooth coefficients. Therefore, smoothness of $u$ follows from the general theory of elliptic PDE (see for example [68]). Other consequences are the maximum principle, the local Harnack inequality and the convergence principles.

The standard way of constructing harmonic functions is by solving the Dirichlet problem. If $B$ is a precompact open set on $M$ with smooth boundary, then, for any continuous function $f$ on $\partial B$, there exists a unique function $u \in C(\bar{B}) \cap C^{2}(B)$ such that

$$
\left\{\begin{array}{l}
\Delta u=0 \\
\left.u\right|_{\partial B}=f .
\end{array}\right.
$$

This is proved exactly in the same way as the solvability of the Dirichlet problem for elliptic equations in bounded regions of $\mathbb{R}^{d}$, for example, by constructing a weak solution and then proving its regularity or by constructing the Perron solutions.

Alternatively, the solution of (4.2) is given by the formula of Kakutani,

$$
u(x)=\mathbb{E}_{x}\left(f\left(X_{\tau}\right)\right),
$$

where $\tau$ is the first hitting time of the boundary $\partial B$ by the process $X_{t}$.

The Green formula (2.6) implies that, for a harmonic function $u$ and for any precompact open set $\Omega$ in the domain of $u$, the flux of $u$ through the boundary $\partial \Omega$ is zero, that is

$$
\operatorname{flux}_{\partial \Omega} u:=\int_{\partial \Omega} \frac{\partial u}{\partial \nu} d \mu^{\prime}=0
$$


where $\nu$ is the outward normal unit vector field on $\partial \Omega$ (assuming $\partial \Omega$ is smooth enough). Moreover, (4.3) is equivalent to harmonicity of $u$. Indeed, it implies by $(2.6)$

$$
\int_{\Omega} \Delta u d \mu=0
$$

for any $\Omega$ in the domain of $u$, whence $\Delta u=0$.

A function $s$ defined in the region $\Omega \subset M$ is called superharmonic if $s$ is continuous $^{2}$ and if, for any precompact region $U \subset \subset \Omega$ and any harmonic function $u \in C^{2}(U) \cap C(\bar{U}), s \geq u$ on $\partial U$ implies $s \geq u$ on $U$. If $s \in C^{2}(\Omega)$ then the superharmonicity of $s$ is equivalent to

$$
\Delta s \leq 0,
$$

which easily follows from the maximum principle. Conversely, if $s \in C(\Omega)$ and (4.4) holds in the sense of distributions, then $s$ is superharmonic.

Let us mention the following simple properties of superharmonic functions.

1. If $\left\{\Omega_{\alpha}\right\}$ is a family of open sets and the function $s$ is superharmonic in each $\Omega_{\alpha}$, then $s$ is superharmonic in their union $\bigcup_{\alpha} \Omega_{\alpha}$.

2. The minimum of two superharmonic functions is also superharmonic.

A function $u$ is subharmonic if $-u$ is superharmonic.

4.2. Green function. As soon as we have constructed the heat kernel, the easiest way to introduce the Green function $G(x, y)$ is to set

$$
G(x, y):=\frac{1}{2} \int_{0}^{\infty} p(t, x, y) d t .
$$

The factor $\frac{1}{2}$ appears because the heat kernel is generated by $\frac{1}{2} \Delta$ rather than by $\Delta$.

An independent definition is as follows: $G(x, y)$ is the smallest positive fundamental solution of the Laplace equation on $M$. We follow the convention that $G \equiv+\infty$ if there is no positive fundamental solution, which matches the case when the integral in (4.5) diverges. If $G \not \equiv \infty$ then we have, for any fixed $y$,

$$
\Delta G(\cdot, y)=-\delta_{y}
$$

For example, in $\mathbb{R}^{d}, d>2$, the Green function is given by

$$
G(x, y)=\frac{c_{d}}{|x-y|^{d-2}},
$$

where $c_{d}=\left(\omega_{d}(d-2)\right)^{-1}$. In $\mathbb{R}^{2}$, we have $G \equiv \infty$ (indeed, the fundamental solution $\log |x-y|$ is signed).

Yet another way of constructing $G$ (which will be most useful for our purposes) is by using an exhaustion sequence. A sequence $\left\{\mathcal{E}_{k}\right\}$ of sets in $M$ is called an exhaustion sequence if

- each $\mathcal{E}_{k}$ is a precompact region with a smooth boundary;

- $\mathcal{E}_{k} \subset \subset \mathcal{E}_{k+1}$;

- $\bigcup_{k} \mathcal{E}_{k}=M$.

\footnotetext{
${ }^{2}$ Sometimes it is useful to relax the continuity of $s$ as to the lower semi-continuity. However, we will not use lower-semicontinuous superharmonic functions here.
} 
One first constructs in each $\mathcal{E}_{k}$ a Green function $G_{\mathcal{E}_{k}}(x, y)$ of the Dirichlet problem in $\mathcal{E}_{k}$, which is continuous up to the boundary $\partial \mathcal{E}_{k}$ (as a function of $x$, for any $\left.y \in \mathcal{E}_{k}\right)$ and vanishes on $\partial \mathcal{E}_{k}$. By the maximum principle, the sequence $\left\{G_{\mathcal{E}_{k}}\right\}$ increases in $k$. The limit as $k \rightarrow \infty$ (finite or infinite) is the global Green function $G(x, y)$. It is easy to see that this limit is independent of the choice of the exhaustion sequence. This construction is justified in [122].

If $M$ is a manifold with boundary, then the Green functions $G$ and $G_{\Omega}$ are assumed to satisfy the Neumann boundary condition on $\partial M$ and $\partial M \cap \Omega$, respectively.

The following properties of $G(x, y)$ will be frequently used.

1. The Green function $G(x, y)$ is either finite for all $x \neq y$ or infinite for all $x, y$. In the former case, we will say that $G$ is finite. The on-diagonal value $G(x, x)$ is always infinite. Moreover, the singularity of $G(x, y)$ as $x \rightarrow y$ is of the same order as that in $\mathbb{R}^{d}$, that is,

$$
G(x, y) \asymp\left\{\begin{array}{ll}
r^{2-d}, & d>2, \\
\log \frac{1}{r}, & d=2,
\end{array} \quad \text { as } r:=\operatorname{dist}(x, y) \rightarrow 0 .\right.
$$

2. Positivity: $G(x, y)>0$.

3. Symmetry: $G(x, y)=G(y, x)$.

4. $G(\cdot, y)$ is harmonic away from $y$ (in fact, $G(\cdot, y)$ is superharmonic on $M$ if one allows $+\infty$ as a value of the function).

5. If $\Omega$ is a precompact region with smooth boundary, then the flux of $G(\cdot, y)$ through $\partial \Omega$ is equal to -1 if $y \in \Omega$ and equal to 0 if $y \notin \bar{\Omega}$, that is

$$
\operatorname{flux}_{\partial \Omega} G=\int_{\partial \Omega} \frac{\partial G(x, y)}{\partial \nu} d \mu^{\prime}(x)= \begin{cases}-1, & y \in \Omega, \\ 0, & y \notin \bar{\Omega},\end{cases}
$$

where $\nu$ denotes the outward unit normal vector field on $\partial \Omega$. Moreover, (4.7) is equivalent to the fact that $G$ is a fundamental solution. The second line in (4.7) follows from the harmonicity of $G$ away from $y$ (cf. (4.3)), whereas the first one reflects the fact that $\Delta G=-\delta_{y}$.

6. A consequence of the minimality:

$$
\inf _{x \in M} G(x, y)=0 .
$$

Example 4.1. Let $M_{\sigma}$ be a spherically symmetric manifold with the pole $o$. Let us prove that the Green function $G(x, o)$ at $o$ can be computed as follows:

$$
G(x, o)=\int_{\rho}^{\infty} \frac{d r}{S(r)},
$$

where $\rho=\operatorname{dist}(x, o)$ (assuming that the integral in (4.8) converges).

To that end, let us first consider the function

$$
v(\rho)=\int \frac{d \rho}{S(\rho)}
$$

(we take the indefinite integral here). We claim that $v(\rho)$ is a harmonic function on $M \backslash\{o\}$ assuming that $\rho$ is the polar radius. Indeed, (4.9) implies that $v$ satisfies the following ODE:

$$
v^{\prime \prime}+\frac{S^{\prime}}{S} v^{\prime}=0
$$

(in fact, (4.9) was found to solve (4.10)). On the other hand, by (3.7), equation (4.10) is the radial part of the Laplace equation. Thus, $\Delta v=0$. 
Moreover, the flux of $v$ through any sphere $\partial B(o, r)$ is equal to 1 . Indeed, (4.9) implies $v^{\prime}=\frac{1}{S(\rho)}$ whence

$$
\operatorname{flux}_{\partial B(o, r)} v=\int_{\partial B(o, r)} \frac{\partial v}{\partial \nu} d \mu^{\prime}=v^{\prime}(r) S(r)=1 .
$$

Thus, the function

$$
\int_{\rho}^{\infty} \frac{d r}{S(r)}
$$

is harmonic away from $o$, has the flux -1 through any sphere $\partial B(o, r)$ and vanishes at $\rho=\infty$, which implies that it coincides with the Green function $G(o, x)$.

4.3. Capacity. Let $\Omega$ be an open set on $M$ and $K$ be a compact set in $\Omega$. We call the pair $(K, \Omega)$ a capacitor and define the capacity $\operatorname{cap}(K, \Omega)$ by

$$
\operatorname{cap}(K, \Omega)=\inf _{\phi \in \mathcal{L}(K, \Omega)} \int_{\Omega}|\nabla \phi|^{2} d \mu,
$$

where $\mathcal{L}(K, \Omega)$ is a set of locally Lipschitz functions $\phi$ on $M$ with a compact support in $\bar{\Omega}$ such that $0 \leq \phi \leq 1$ and $\left.\phi\right|_{K}=1$.

For an open precompact set $K \subset \Omega$, we define its capacity by

$$
\operatorname{cap}(K, \Omega):=\operatorname{cap}(\bar{K}, \Omega) \text {. }
$$

Therefore, definition (4.12) can be applied in this case too, since $\left.\phi\right|_{K}=1$ is equivalent to $\left.\phi\right|_{K}=1$. It is possible to define the capacity when $K$ is a Borel set, but we do not need that (see [29] and [134, Section 2.2]). Since $\nabla \phi=0$ on $\bar{K}$, we see that the capacity $\operatorname{cap}(K, \Omega)$ is determined by the intrinsic properties of $\Omega \backslash \bar{K}$.

If $\Omega=M$ then we write $\operatorname{cap}(K)$ for $\operatorname{cap}(K, \Omega)$. It is obvious from the definition that the set $\mathcal{L}(K, \Omega)$ increases on expansion of $\Omega$ (and on shrinking of $K$ ). Therefore, the capacity $\operatorname{cap}(K, \Omega)$ decreases on expanding of $\Omega$ (and on shrinking of $K$ ). In particular, one can prove that, for any exhaustion sequence $\left\{\mathcal{E}_{k}\right\}$,

$$
\operatorname{cap}(K):=\lim _{k \rightarrow \infty} \operatorname{cap}\left(K, \mathcal{E}_{k}\right) .
$$

Let $\Omega$ be precompact. It is well known that the Dirichlet integral in (4.12) is minimized by a harmonic function. Therefore, the infimum in (4.12) is attained at the function $\phi=u$ which is the (Perron) solution to the following Dirichlet problem in $\Omega \backslash K$ :

$$
\left\{\begin{array}{l}
\Delta u=0 \\
\left.u\right|_{\partial \Omega}=0 \\
\left.u\right|_{\partial K}=1 .
\end{array}\right.
$$

The function $u$ is called the equilibrium potential or the capacity potential of the capacitor $(K, \Omega)$.

It is obvious that if the boundaries of $\Omega$ and $K$ are smooth enough, then $u \in$ $\mathcal{L}(K, \Omega)$. We have then, by the Green formula (2.6) and (4.13),

$$
\begin{aligned}
\operatorname{cap}(K, \Omega) & =\int_{\Omega}|\nabla u|^{2} d \mu=\int_{\Omega \backslash K}|\nabla u|^{2} d \mu \\
& =-\int_{\Omega \backslash K} u \Delta u d \mu+\int_{\partial K \cup \partial \Omega} \frac{\partial u}{\partial \nu} u d \mu^{\prime} \\
& =\int_{\partial K} \frac{\partial u}{\partial \nu} d \mu^{\prime}=-\operatorname{flux}_{\partial K} u
\end{aligned}
$$




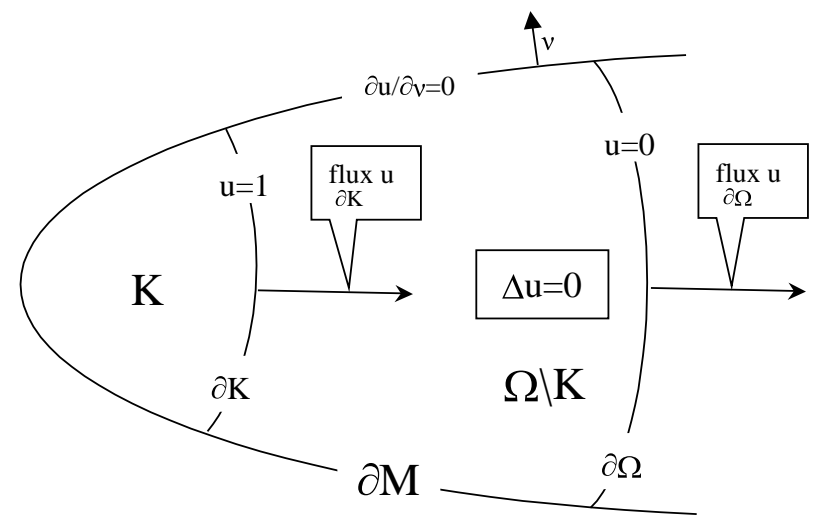

Figure 6 . The capacity potential for the capacitor $(K, \Omega)$ on a manifold with boundary

where $\nu$ is the outward unit normal vector field on $\partial(\Omega \backslash K)$ (the negative sign in (4.14) appears because $\nu$ points inward to $K$ ). On the other hand, the harmonicity of $u$ and (4.3) imply

$$
0=\operatorname{flux}_{\partial(\Omega \backslash K)} u=\operatorname{flux}_{\partial \Omega} u-\operatorname{flux}_{\partial K} u .
$$

Identities (4.14) and (4.15) imply the following formulas of the capacity:

$$
\operatorname{cap}(K, \Omega)=\int_{\Omega}|\nabla u|^{2} d \mu=-\operatorname{flux}_{\partial K} u=-\operatorname{flux}_{\partial \Omega} u .
$$

In general, despite the fact that $u$ may not be in $\mathcal{L}(K, \Omega)$, the Dirichlet integral of $u$ is still equal to the capacity.

It is useful to know that various classes of test functions in the definition of capacity may be allowed without changing the value of the capacity. For example, the class $\mathcal{L}$ in (4.12) can be replaced by the following class $\mathcal{D}$ :

$$
\mathcal{D}(K, \Omega):=\left\{\phi \in C_{0}^{\infty}(\Omega): 0 \leq \phi \leq 1 \text { and } \phi=1 \text { in a neighbourhood of } K\right\} .
$$

If $M$ is a manifold with boundary, then all the above remain true, with the additional property that the capacity potential $u$ of $(K, \Omega)$ should satisfy the Neumann boundary condition on $\partial M \cap(\Omega \backslash K)$ should the latter be non-empty. If $M$ is made of a conducting material, then a physical meaning of $\operatorname{cap}(K, \Omega)$ is a conductivity of the piece of $M$ between $\partial K$ and $\partial \Omega$. Put differently, the flux of $u$ through $\partial K$ and $\partial \Omega$ is equal to the current through $M$ provided the potential difference between $\partial K$ and $\partial \Omega$ is equal to 1 (see Figure 6 ).

Given an open set $E \subset M$, one can define the capacity relative to $E$ as follows:

$$
\operatorname{cap}_{E}(K, \Omega)=\inf _{\phi \in \mathcal{L}(K, \Omega)} \int_{\Omega \cap E}|\nabla \phi|^{2} d \mu,
$$

where $(K, \Omega)$ is a capacitor on $M$ as above. The difference between (4.18) and (4.12) is that the integral in the former is taken over $\Omega \cap E$ rather than over $\Omega$. Clearly, the capacity $\operatorname{cap}_{E}(K, \Omega)$ does not depend on the geometry away from $\bar{E}$. If $\Omega=M$ then we write $\operatorname{cap}_{E}(K)$ for $\operatorname{cap}_{E}(K, \Omega)$. 
If $\partial E$ is smooth enough, then $\bar{E}$ can be considered as a manifold with boundary. In this case the relative capacity $\operatorname{cap}_{E}$ coincides with the capacity cap $\bar{E}$ on the manifold $\bar{E}$, in the following sense:

$$
\operatorname{cap}_{E}(K, \Omega)=\operatorname{cap}_{\bar{E}}(K \cap \bar{E}, \Omega \cap \bar{E}) .
$$

Example 4.2. Let us show how to compute the capacity cap $(B(o, r), B(o, R))$ on the model manifold $M_{\sigma}$ where $o$ is the pole of $M_{\sigma}$ and $0<r<R$. The function

$$
u(\rho, \theta)=u(\rho)=a \int_{\rho}^{R} \frac{d \xi}{S(\xi)}
$$

is the capacity potential of the capacitor $(B(o, r), B(o, R))$ where the constant $a$ is chosen to ensure $u(r)=1$, i.e.

$$
a=\left(\int_{r}^{R} \frac{d \xi}{S(\xi)}\right)^{-1}
$$

Since by (4.11)

$$
\operatorname{flux}_{\partial B(o, R)} u=-a
$$

we conclude by (4.16) that

$$
\operatorname{cap}(B(o, r), B(o, R))=\left(\int_{r}^{R} \frac{d \xi}{S(\xi)}\right)^{-1}
$$

and

$$
\operatorname{cap}(B(o, r))=\left(\int_{r}^{\infty} \frac{d \xi}{S(\xi)}\right)^{-1}
$$

In particular, in $\mathbb{R}^{d}$ we have

$$
\operatorname{cap}(B(o, r))= \begin{cases}c_{d} r^{d-2}, & d>2 \\ 0, & d=2 .\end{cases}
$$

The following statement establishes a useful link between capacity and the Green function.

Proposition 4.1. ([127], [71]) Let $U$ be an open precompact set in $M$ and $y \in U$. Then the following inequality is true:

$$
\inf _{x \in \partial U} G(x, y) \leq \operatorname{cap}(U)^{-1} \leq \sup _{x \in \partial U} G(x, y) .
$$

Furthermore, if $\Omega$ is a precompact set in $M$ with a smooth boundary and $\Omega \supset \bar{U}$, then

$$
\inf _{x \in \partial U} G_{\Omega}(x, y) \leq \operatorname{cap}(U, \Omega)^{-1} \leq \sup _{x \in \partial U} G_{\Omega}(x, y) .
$$

Proof. Since (4.24) follows from (4.25) by letting $\Omega \uparrow M$, it suffices to prove (4.25). Let us set

$$
a:=\max _{x \in \partial U} G_{\Omega}(x, y) \quad \text { and } \quad b:=\min _{x \in \partial U} G_{\Omega}(x, y) .
$$

For any number $c$, let us define

$$
F_{c}:=\left\{x \in \Omega: G_{\Omega}(x, y) \geq c\right\} .
$$




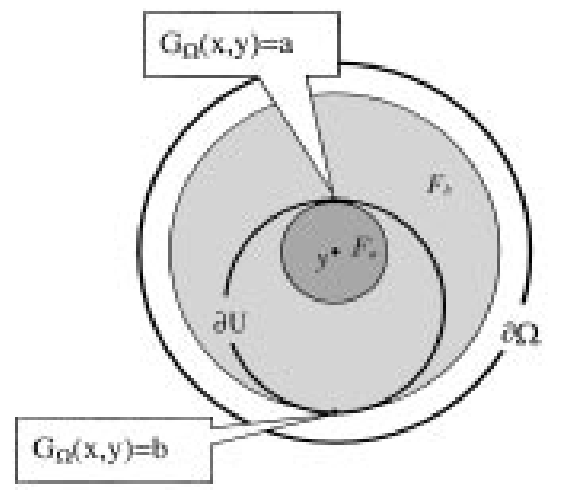

Figure 7 . Sets $F_{a}$ and $F_{b}$

We claim that

$$
F_{a} \subset \bar{U} \subset F_{b} .
$$

Indeed, the function $G_{\Omega}(\cdot, y)$ is harmonic in $\Omega \backslash \bar{U}$, and, by the maximum principle, its supremum in $\Omega \backslash \bar{U}$ is attained on the boundary $\partial(\Omega \backslash \bar{U})=\partial \Omega \cup \partial U$. Since $G_{\Omega}$ vanishes on $\partial \Omega$, we have

$$
\sup _{x \in \Omega \backslash \bar{U}} G_{\Omega}(x, y)=\max _{x \in \partial U} G_{\Omega}(x, y)=a,
$$

whence $F_{a} \subset \bar{U}$. Similarly, the function $G_{\Omega}(\cdot, y)$ is superharmonic in $U$, whence, by the minimum principle,

$$
\inf _{x \in U} G_{\Omega}(x, y)=\min _{x \in \partial U} G_{\Omega}(x, y)=b
$$

and $F_{b} \supset \bar{U}$ (see Figure 7).

The inclusions (4.26) imply

$$
\operatorname{cap}\left(F_{a}, \Omega\right) \leq \operatorname{cap}(U, \Omega) \leq \operatorname{cap}\left(F_{b}, \Omega\right),
$$

whence (4.25) will follow if we show that, for any $c>0$ (in particular, for $c=a$ and $c=b)$,

$$
\operatorname{cap}\left(F_{c}, \Omega\right)=\frac{1}{c} .
$$

Indeed, the function $u:=\frac{1}{c} G_{\Omega}(\cdot, y)$ is the equilibrium potential of the capacitor $\left(F_{c}, \Omega\right)$. Therefore, by (4.16) and (4.7),

$$
\operatorname{cap}\left(F_{c}, \Omega\right)=-\operatorname{flux}_{\partial \Omega} u=-\frac{1}{c} \operatorname{flux}_{\partial \Omega} G_{\Omega}(\cdot, y)=\frac{1}{c},
$$

which was to be proved.

4.4. Massive sets. The following notion of massiveness will play an important role in the sequel.

Definition 4.1. Given an open set $\Omega \subset M$, we say that a function $v \geq 0$ is an admissible subharmonic function for $\Omega$ if it is a bounded subharmonic function on $M$ such that $v=0$ in $M \backslash \Omega$ and $\sup _{\Omega} v>0$ (see Figure 8). An open set $\Omega$ is called massive if there is at least one admissible subharmonic function for $\Omega$. Alternatively, $\Omega$ is massive if there exists an admissible superharmonic function $u$ 


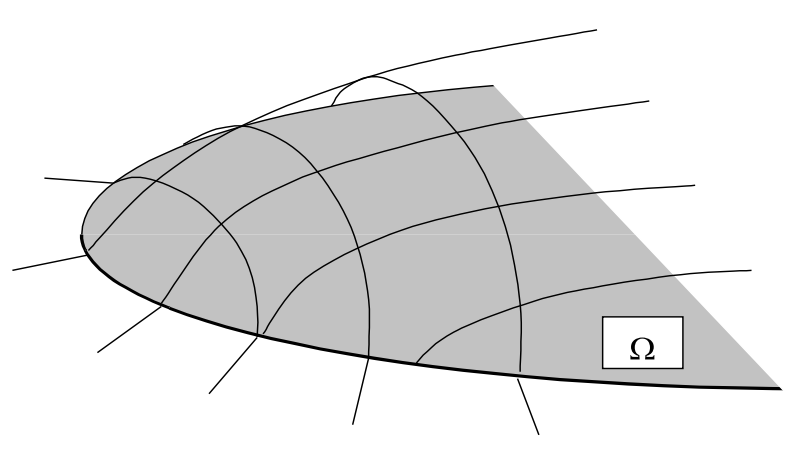

Figure 8. Admissible subharmonic function $v$ for $\Omega$

for $\Omega$, i.e. a bounded superharmonic function $u \geq 0$ on $M$ such that $u \equiv 1$ outside $\Omega$ and $\inf _{\Omega} u=0$.

We say that an open set $\Omega$ is $D$-massive if there is an admissible subharmonic function $v$ for $\Omega$ (or an admissible superharmonic function $u$ ) which has a finite Dirichlet integral:

$$
\int_{M}|\nabla v|^{2} d \mu<\infty
$$

Clearly, massiveness is an intrinsic property of $\Omega$, despite the function $v$ being formally defined on the whole $M$. The manifold itself is always massive because the constant function is an admissible subharmonic function. The empty set is always non-massive.

We say that an open set $\Omega$ is proper if $\bar{\Omega} \neq M$. By the maximum principle, a proper open precompact set is never massive.

Proposition 4.2. Massiveness (D-massiveness) is preserved by increasing the set $\Omega$, as well as by reducing it by a compact (for the latter, we assume that $\Omega$ is proper).

Proof. If $\Omega^{\prime} \supset \Omega$ then any admissible subharmonic function $v$ for $\Omega$ is also admissible subharmonic for $\Omega^{\prime}$. If $\Omega^{\prime}=\Omega \backslash K$ where $K$ is a compact, then the function $v^{\prime}:=(v-c)_{+}$, where $c:=\sup _{K} v$, is admissible subharmonic for $\Omega^{\prime}$. Indeed, we need only to show that $\sup v^{\prime}>0$. The fact that $\Omega$ is proper implies $v \not \equiv$ const. Therefore, by the strong maximum principle, $c<\sup _{\Omega} v$, whence $\sup v^{\prime}>0$.

Let us show some examples of massive and non-massive sets. Note that in $\mathbb{R}^{2}$, all proper open subsets are non-massive because there is no bounded subharmonic function except for the constant function.

Example 4.3. The exterior $\Omega$ of ball $\overline{B(o, 1)}$ in $\mathbb{R}^{d}, d>2$, is massive. Indeed, the function

$$
u(x)= \begin{cases}|x|^{2-d}, & |x|>1 \\ 1, & |x| \leq 1\end{cases}
$$

is an admissible superharmonic function for $\Omega$. Moreover, $u$ has finite Dirichlet integral so that $\Omega$ is $D$-massive. 


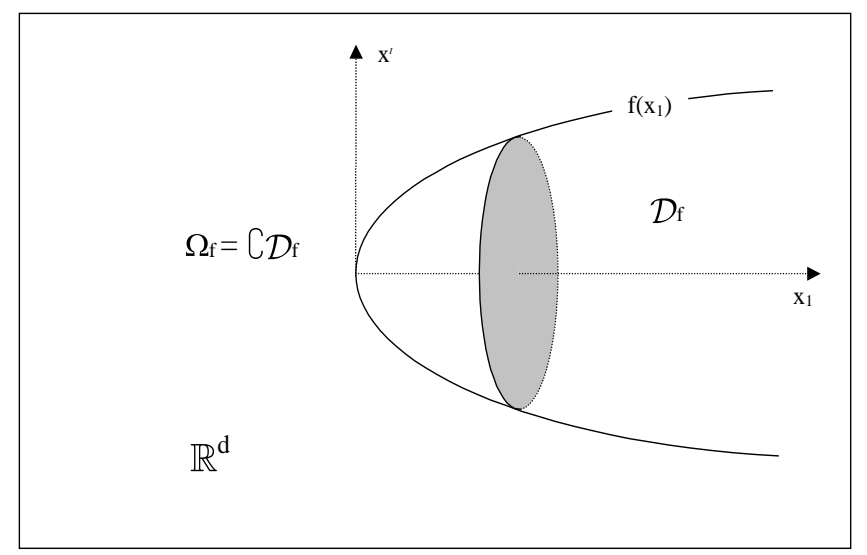

FiguRE 9 . Set $\Omega_{f}$ is the exterior of the domain of revolution $\mathcal{D}_{f}$

Example 4.4. A half-space in $\mathbb{R}^{d}$ is non-massive - a simple proof of that will be given below (see Example 4.6). Similarly, any cone $\mathrm{e}^{3}$ in $\mathbb{R}^{d}$ is non-massive. On the contrary, any angle on the hyperbolic plane $\mathbb{H}^{2}$ is $D$-massive (see [73, Section 3]). Any cone in $\mathbb{H}^{d}$ is massive but not $D$-massive unless $d=2$ (see [73, Section 5]).

Example 4.5. Consider a domain of revolution in $\mathbb{R}^{d}$

$$
\mathcal{D}_{f}:=\left\{x \in \mathbb{R}^{d}: 0 \leq x_{1}<\infty \text { and }\left|x^{\prime}\right| \leq f\left(x_{1}\right)\right\}
$$

where $x^{\prime}=\left(x_{2}, \ldots, x_{d}\right)$ and $f$ is a smooth function possessing certain regularity (see Figure 9). Denote $\Omega_{f}=\mathbb{R}^{d} \backslash \mathcal{D}_{f}$ and $\alpha=1 /(d-3)$, assuming $d>3$. Then $\Omega_{f}$ is massive if $f(t)=t \log ^{-(\alpha+\varepsilon)} t(t$ is large and $\varepsilon>0)$ and is not massive if $f(t)=t \log ^{-\alpha} t$. Moreover, $\Omega_{f}$ is $D$-massive if $f(t)=t^{-(\alpha+\varepsilon)}$ and is not $D$-massive if $f(t)=t^{-\alpha}$. See [85, Proposition 6.3] for a criterion of massiveness of $\Omega_{f}$ and [73, Section 3] for a criterion for $D$-massiveness of $\Omega_{f}$.

Definition 4.2. The subharmonic potential $b_{\Omega}$ of an open set $\Omega$ is the supremum of all admissible subharmonic functions $v$ for $\Omega$ such that $v \leq 1$. The superharmonic potential $s_{\Omega}$ of $\Omega$ is the infimum of all admissible superharmonic functions for $\Omega$.

If there is no admissible subharmonic (superharmonic) function, then we naturally let $b_{\Omega} \equiv 0$ (respectively, $s_{\Omega} \equiv 1$ ). It is obvious that always

$$
s_{\Omega}+b_{\Omega}=1,
$$

and the function $b_{\Omega}$ is increasing on expansion of $\Omega$ whereas $s_{\Omega}$ is decreasing. Clearly, $\Omega$ is massive if and only if $b_{\Omega} \not \equiv 0$ and $s_{\Omega} \not \equiv 1$.

The function $b_{\Omega}$ is called also the harmonic measure of the set $F:=M \backslash \Omega$. Another term for $s_{\Omega}$ is the reduced (or reduit) function of $F$. Let us emphasize that the functions $b_{\Omega}, s_{\Omega}$ are determined by the set $\Omega$ intrinsically.

For a set $\Omega$ with smooth boundary, we will construct $b_{\Omega}$ and $s_{\Omega}$ as the limits of solutions of a series of certain Dirichlet problems. Choose an exhaustion $\left\{\mathcal{E}_{k}\right\}$ of $M$ so that the boundaries $\partial \mathcal{E}_{k}$ and $\partial \Omega$ are transversal, and solve, for any set $\Omega \cap \mathcal{E}_{k}$,

${ }^{3}$ By "a cone" we always mean an infinite cone with a compact base. 


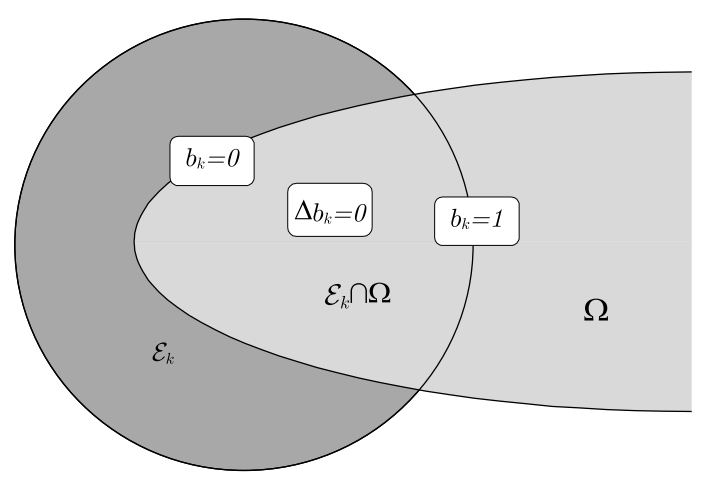

FIGURE 10. Function $b_{k}$

the following Dirichlet problem (see Figure 10)

$$
\left\{\begin{array}{l}
\Delta b_{k}=0 \\
\left.b_{k}\right|_{\partial \Omega \cap \mathcal{E}_{k}}=0 \\
\left.b_{k}\right|_{\partial \mathcal{E}_{k} \cap \Omega}=1 .
\end{array}\right.
$$

Proposition 4.3. Let $\Omega \subset M$ be a non-empty open set.

(i) Assume that $\Omega$ has non-empty smooth boundary. Then

$$
b_{\Omega}=\lim _{k \rightarrow \infty} b_{k} \quad \text { in } \Omega .
$$

The function $b_{\Omega}$ is continuous, subharmonic on $M$ and harmonic in $\Omega$. Respectively, the function $s_{\Omega}$ is continuous, superharmonic on $M$ and harmonic in $\Omega$.

(ii) For any proper open set $\Omega$, we have

$$
b_{\Omega}=\sup _{\Omega^{\prime}} b_{\Omega^{\prime}}
$$

where the supremum is taken over all regions $\Omega^{\prime}$ with smooth boundaries whose closure is contained in $\Omega$.

(iii) The following dichotomy takes place:

either $\Omega$ is non-massive, $b_{\Omega} \equiv 0$ and $s_{\Omega}=1$

or $\Omega$ is massive, $\sup b_{\Omega}=1$ and $\inf s_{\Omega}=0$.

Proof. (i) By the maximum principle, the sequence $\left\{b_{k}\right\}$ is decreasing. The limit $b_{\infty}:=\lim _{k \rightarrow \infty} b_{k}$ is harmonic in $\Omega$, continuous up to $\partial \Omega$ and vanishes on $\partial \Omega$. Let us extend $b_{\infty}$ by setting it equal to 0 in $M \backslash \bar{\Omega}$. We claim that $b_{\infty}=b_{\Omega}$. Indeed, the function $b_{\infty}$ is obviously a continuous subharmonic function, $0 \leq b_{\infty} \leq 1$, and $b_{\infty}$ vanishes outside $\Omega$. If there is another function $v$ possessing these properties, then by the maximum principle, $v \leq b_{k}$ in $\mathcal{E}_{k} \cap \Omega$, whence $v \leq b_{\infty}$. Hence, $b_{\infty}$ is the supremum of all admissible subharmonic functions $v$ such that $v \leq 1$, that is, $b_{\infty}=b_{\Omega}$ (see Figure 11).

Note that if $\partial \Omega$ is not smooth, then $b_{\infty}$ may be discontinuous at irregular points of the boundary $\partial \Omega$.

(ii) Since $b_{\Omega} \geq b_{\Omega^{\prime}}$, we have only to show that

$$
b_{\Omega} \leq \sup _{\Omega^{\prime}} b_{\Omega^{\prime}} .
$$






FIGURE 11. Sequence of $b_{k}$

For any open $\Omega^{\prime}$ such that $\overline{\Omega^{\prime}} \subset \Omega$, there exists an open set $\Omega^{\prime \prime}$ with smooth boundary such that $\Omega^{\prime} \subset \Omega^{\prime \prime}$ and $\overline{\Omega^{\prime \prime}} \subset \Omega$, whence $b_{\Omega^{\prime}} \leq b_{\Omega^{\prime \prime}}$. Therefore, it suffices to prove (4.28) without the requirement that $\partial \Omega^{\prime}$ is smooth. Let $v$ be any admissible subharmonic function for $\Omega$. Take any $\varepsilon \in(0, \sup v)$ and consider the set $\Omega^{\prime}=$ $\{v>\varepsilon\}$. Since $(v-\varepsilon)_{+}$is an admissible subharmonic function for $\Omega^{\prime}$, we have

$$
(v-\varepsilon)_{+} \leq b_{\Omega^{\prime}} .
$$

By taking sup over $v$ and $\varepsilon$, we obtain (4.28).

(iii) By definition, $b_{\Omega} \not \equiv 0$ is equivalent to the massiveness of $\Omega$. Let us show that $b_{\Omega} \not \equiv 0$ implies $\sup b_{\Omega}=1$. If $\sup b_{\Omega}=: c<1$ then, for any admissible subharmonic function $v$, we have $\sup v \leq c$. However, then the function $c^{-1} v$ is also admissible subharmonic, whence, by the definition of $b_{\Omega}$, we obtain $c^{-1} v \leq b_{\Omega}$ and $\sup b_{\Omega} \geq 1$.

Example 4.6. Let us show that a half-space $\Omega$ in $\mathbb{R}^{d}$ is non-massive. Consider the function $u$ which is equal to $b_{\Omega}$ on $\Omega$ and is extended oddly over the boundary $\partial \Omega$ to the whole space. Since $u$ is harmonic in $\mathbb{R}^{d}$ and bounded, the Liouville theorem implies $u \equiv$ const and, hence, $u \equiv 0$. Therefore, $b_{\Omega} \equiv 0$, and $\Omega$ is non-massive.

4.5. Hitting probabilities. In this section, we compute, in terms of the function $s_{\Omega}$, the following probabilities.

1. The $\mathbb{P}_{x}$-probability that the Brownian motion $X_{t}$ visits a set $F \subset M$ ever. Denote it by

$$
e_{F}(x):=\mathbb{P}_{x}\left\{\exists t \geq 0 \quad \text { such that } X_{t} \in F\right\} .
$$

In the potential-theoretic language, the function $e_{F}$ is called the reduit function of $F$ and is denoted by $R_{F}^{1}$.

2. The $\mathbb{P}_{x}$-probability that the Brownian motion $X_{t}$ hits $F$ at a sequence of arbitrarily large times. Denote it by

$$
h_{F}(x):=\mathbb{P}_{x}\left\{\exists\left\{t_{k}\right\} \text { such that } t_{k} \rightarrow \infty \text { and } X_{t_{k}} \in F, \text { for all } k \in \mathbb{N}\right\} .
$$

Proposition 4.4. Let $\Omega \subset M$ be a non-empty open set with smooth boundary, and denote $F:=M \backslash \Omega$.

(i) (G.A.Hunt) For any $x \in M$, we have

$$
e_{F}(x)=s_{\Omega}(x) .
$$




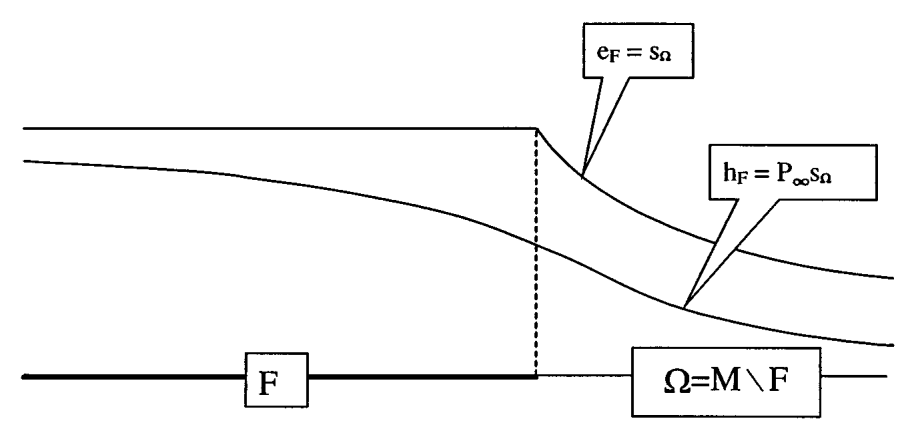

FIGURE 12. The hitting probabilities $e_{F}$ and $h_{F}$

(ii) Let us denote

$$
P_{\infty} s_{\Omega}:=\lim _{t \rightarrow \infty} P_{t} s_{\Omega}(x) .
$$

Then, for any $x \in M$,

$$
h_{F}(x)=P_{\infty} s_{\Omega}(x)
$$

Remark. The function $b_{\Omega}(x)$ is also called the escape function of $\Omega$ because, due to (i), $b_{\Omega}(x)=1-e_{F}(x)$ which is equal to the $\mathbb{P}_{x}$-probability of $X_{t}$ escaping to infinity within $\Omega$, without touching $\partial \Omega{ }^{4}$

Remark. The function $u:=P_{\infty} s_{\Omega}$ is harmonic on $M$ because $P_{t} u=u$. Thus, $h_{F}(x)$ is a harmonic function of $x$ on all of $M$. Let us recall for comparison that $e_{F}=s_{\Omega}$ is harmonic in $\Omega$ but is superharmonic in $M$ (see Figure 12).

Remark. Assertion (i) implies that massiveness has the following probabilistic meaning: the set $\Omega$ is massive if and only if $e_{F}(x) \not \equiv 1$.

Proof. (i) If $x \in F$ then $e_{F}(x)=1=s_{\Omega}(x)$, and there is nothing to prove.

Now let $x \in \Omega$. Choose an exhaustion sequence $\left\{\mathcal{E}_{k}\right\}$, and consider the event $\mathcal{A}_{k}$ that the trajectory $X_{t}$ hits the boundary $\partial \Omega$ before $\partial \mathcal{E}_{k}$ (see Figure 13). Clearly, the sequence of events $\left\{\mathcal{A}_{k}\right\}$ is expanding, and their union is the event to hit $\partial \Omega$ (and thus $F$ ) ever, whence

$$
e_{F}(x)=\lim _{k \rightarrow \infty} \mathbb{P}_{x}\left(\mathcal{A}_{k}\right)
$$

On the other hand, let $f_{k}$ be a function on $\partial\left(\mathcal{E}_{k} \cap \Omega\right)$ which is equal to 1 on $\partial \Omega$ and 0 on $\partial \mathcal{E}_{k}$, and let $\tau$ denote the first hitting time of $\partial \Omega$. We have

$$
\mathbb{P}_{x}\left(\mathcal{A}_{k}\right)=\mathbb{E}_{x}\left(f_{k}\left(X_{\tau}\right)\right)=s_{k}(x)
$$

where $s_{k}$ solves the Dirichlet problem in $\mathcal{E}_{k} \cap \Omega$ :

$$
\left\{\begin{array}{l}
\Delta s_{k}=0 \\
\left.s_{k}\right|_{\partial \Omega}=1 \\
\left.s_{k}\right|_{\partial \mathcal{E}_{k}}=0 .
\end{array}\right.
$$

\footnotetext{
${ }^{4}$ One should distinguish the following two events: (1) to never hit $F$, which has the $\mathbb{P}_{x^{-}}$ probability $b_{\Omega} ;(2)$ to stay in $\Omega$ for all $t \geq 0$, which has the $\mathbb{P}_{x}$-probability $P_{\infty}^{\Omega} 1 \leq b_{\Omega}$. The latter may be strictly smaller than the former in the case when the process can reach infinity from within $\Omega$ in a finite time. If the manifold $M$ is stochasically complete, then we do have $P_{\infty}^{\Omega} 1=b_{\Omega}$.
} 


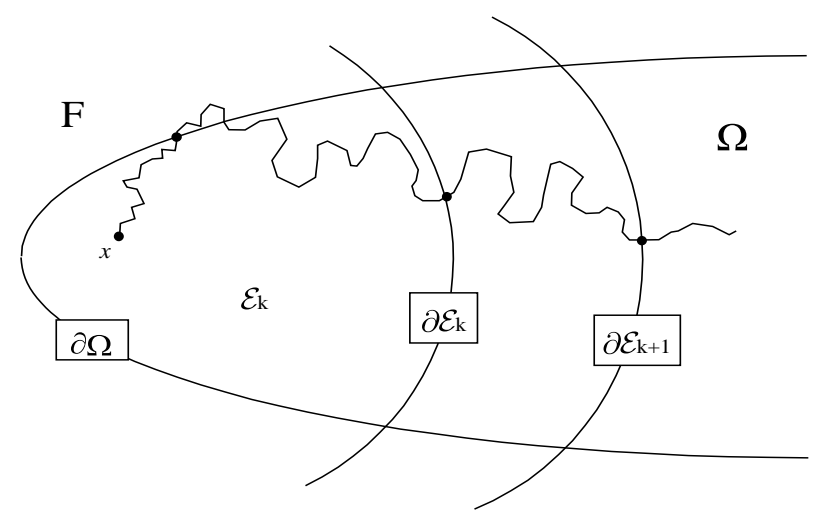

FiguRE 13. Events $\mathcal{A}_{k}$ and $\mathcal{A}_{k+1}$

Since $s_{\Omega}=\lim _{k \rightarrow \infty} s_{k}$ by Proposition 4.3, we conclude from (4.30) and (4.31) that $s_{\Omega}=e_{F}$.

(ii) Denote by $\mathcal{B}_{t}$ the event that $X_{T}$ hits $F$ at some time $T \geq t$. Then we have the following identity

$$
\mathbb{P}_{x}\left(\mathcal{B}_{t}\right)=P_{t} s_{\Omega}(x)=\int_{M} p(t, x, y) s_{\Omega}(y) d \mu(y) .
$$

Indeed, at time $t$, the $\mathbb{P}_{x}$-law of the Brownian particle $y=X_{t}$ is $p(t, x, y) d \mu(y)$. Since the $\mathbb{P}_{y}$-probability of the Brownian motion hitting $F$ is equal to $s_{\Omega}(y)$, we obtain (4.33) by the Markov property.

Obviously, the sequence of events $\left\{\mathcal{B}_{t}\right\}$ is decreasing in $t$ (which implies, in particular, that the limit (4.29) exists), and their intersection is the event $\mathcal{B}_{\infty}$ that the Brownian motion visits $F$ at a sequence of arbitrarily large times. Therefore,

$$
h_{F}(x)=\mathbb{P}_{x}\left(\mathcal{B}_{\infty}\right)=\lim _{t \rightarrow \infty} \mathbb{P}_{x}\left(\mathcal{B}_{t}\right)=P_{\infty} s_{\Omega},
$$

which was to be proved.

4.6. Exterior of a compact. If $\Omega$ is an exterior of a compact $F$ on $M$, then some additional criteria of massiveness of $\Omega$ hold true.

Proposition 4.5. Let $\Omega \subset M$ be an open set with non-empty smooth boundary and let $F:=M \backslash \Omega$ be compact.

(a) The following dichotomy takes place:

either $\Omega$ is not massive, $s_{\Omega} \equiv 1$ and $P_{\infty} s_{\Omega} \equiv 1$, or $\Omega$ is massive, $s_{\Omega} \not \equiv 1$ and $P_{\infty} s_{\Omega}=0$.

(b) We have

$$
\int_{\partial \Omega} \frac{\partial s_{\Omega}}{\partial \nu} d \mu^{\prime}=\operatorname{cap}(F)
$$

where $\nu$ is the outward normal vector field at $\partial \Omega$, and

$$
\int_{M}\left|\nabla s_{\Omega}\right|^{2} d \mu=\operatorname{cap}(F) .
$$

Corollary 4.6. Let $\Omega \subset M$ be an open set with non-empty smooth boundary and let $F:=M \backslash \Omega$ be compact. Then 
(a) $\Omega$ is massive if and only if $\Omega$ is $D$-massive.

(b) $\Omega$ is massive if and only if $\operatorname{cap}(F)>0$.

(c) The massiveness of $\Omega$ is equivalent to $e_{F} \not \equiv 1$ and to $h_{F} \equiv 0$.

Proof of Corollary 4.6. (a) If $\Omega$ is massive, then, by (4.35), $s_{\Omega}$ has finite Dirichlet integral. Thus, $\Omega$ is $D$-massive. The opposite direction is a general fact which follows from the definition of massiveness and $D$-massiveness.

(b) The massiveness of $\Omega$ is equivalent to $s_{\Omega} \not \equiv 1$ which is equivalent to $\operatorname{cap}(F)>$ 0 by (4.34) or (4.35).

(c) This follows immediately from Proposition 4.4 and Proposition 4.5(a).

Proof of Proposition 4.5. (a) Assume that $\Omega$ is non-massive and show that $M$ is stochastically complete, that is, $P_{t} 1 \equiv 1$. If we know that already, then we argue as follows: the non-massiveness of $\Omega$ implies $s_{\Omega} \equiv 1$, whence $P_{\infty} s_{\Omega}=P_{\infty} 1=$ $\lim _{t \rightarrow \infty} P_{t} 1 \equiv 1$.

Let us suppose, on the contrary, that $P_{t} 1 \not \equiv 1$ and consider the functions $v(x, t)=$ $P_{t} 1(x)$ and

$$
w(x):=\int_{0}^{\infty} e^{-t} v(x, t) d t .
$$

It is easy to see that

$$
0<w \leq \int_{0}^{\infty} e^{-t} d t=1
$$

and

$$
\frac{1}{2} \Delta w=\int_{0}^{\infty} e^{-t} \frac{1}{2} \Delta v d t=\int_{0}^{\infty} e^{-t} \frac{\partial v}{\partial t} d t=\left.v e^{-t}\right|_{0} ^{\infty}+\int_{0}^{\infty} e^{-t} v d t=-1+w \leq 0 .
$$

Therefore, $w$ is a positive superharmonic function on $M$. The assumption $P_{t} 1 \not \equiv 1$ implies that, for some $x \in M$, we have $w(x)<1$, whence $\Delta w(x)<0$ and $w \not \equiv$ const. By the strong minimum principle, $\inf _{M} w<\inf _{F} w$. Hence, the function $\left(w / \inf _{F} w\right) \wedge 1$ is superharmonic admissible for $\Omega$ and, thus, $\Omega$ is massive, which contradicts the hypothesis. ${ }^{5}$

Assume now that $\Omega$ is massive and, thus, $s_{\Omega} \not \equiv 1$. Let us set $u=P_{\infty} s_{\Omega}$. Function $u$ is harmonic on $M$ and

$$
0 \leq u \leq s_{\Omega} \leq 1
$$

We need to verify that $u \equiv 0$. Assume on the contrary that $\sup u>0$. Since $\inf u \leq \inf s_{\Omega}=0$ (see Proposition 4.3(iii)), we have $u \not \equiv$ const. By the strong maximum principle and the compactness of $F$,

$$
\sup _{F} u<\sup _{M} u \text {. }
$$

\footnotetext{
${ }^{5}$ The proof of the first part of the assertion (a) of Proposition 4.5 contains the following implication: the explosion $\Longrightarrow$ the existence of a non-constant positive superharmonic function (cf. Corollary 6.4 in Section 6).

Another proof can be obtained by using the strong Markov property of the Brownian motion. Indeed, if $s_{\Omega} \equiv 1$ then, by Proposition 4.4, the probability $e_{F}$ of hitting $F$ is identically equal to 1. By the strong Markov property, the probability $h_{F}$ of hitting $F$ at arbitrary large times is also 1 , whence $P_{\infty} s_{\Omega}=h_{F} \equiv 1$.
} 


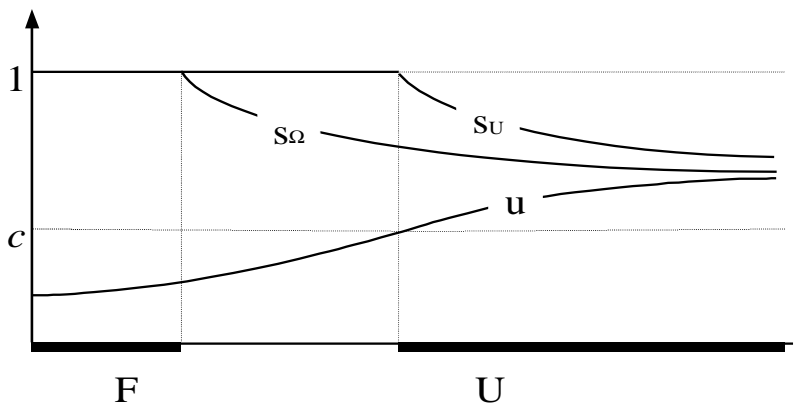

Figure 14. Functions $s_{\Omega}, s_{U}$ and $u$

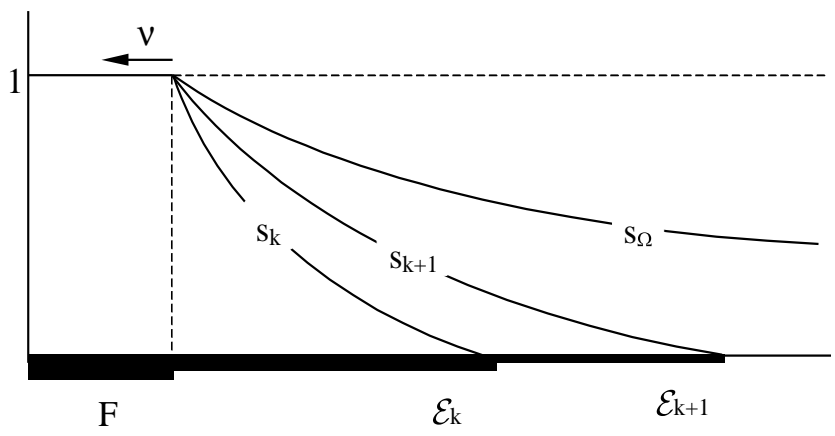

Figure 15. Sequence of $s_{k}$

Choose a number $c$ such that

$$
\sup _{F} u<c<\sup _{M} u
$$

and consider the set $U:=\{u>c\}$. By the choice of $c$, we have $U \subset \Omega$, whence (see Figure 14)

$$
s_{U} \geq s_{\Omega} \geq u .
$$

The set $U$ is massive since the function $(u-c)_{+}$is admissible subharmonic for $U$. By Proposition 4.3, $\inf _{U} s_{U}=0$. However, $\inf _{U} u=c>0$ which contradicts (4.36). ${ }^{6}$

(b) Let $s_{k}$ solve the Dirichlet problem (4.32). Then, for $k$ large enough (such that $\left.\mathcal{E}_{k} \supset F\right)$, the function $s_{k}$ is the equilibrium potential of the capacitor $\left(F, \mathcal{E}_{k}\right)$, whence

$$
\operatorname{cap}\left(F, \mathcal{E}_{k}\right)=\int_{\mathcal{E}_{k} \backslash F}\left|\nabla s_{k}\right|^{2} d \mu=\int_{\partial F} \frac{\partial s_{k}}{\partial \nu} d \mu^{\prime} .
$$

The sequence $\left\{s_{k}\right\}$ is increasing in $k$ and converges to $s_{\Omega}$ (see Figure 15), whence, by the local properties of harmonic functions, all their derivatives converge to those of $s_{\Omega}$ locally uniformly as well. The sequence $\left\{\left.\frac{\partial s_{k}}{\partial \nu}\right|_{\partial F}\right\}$ is decreasing in $k$ because $\left.s_{k}\right|_{\partial F}=1$ and, thus, converges to $\left.\frac{\partial s_{\Omega}}{\partial \nu}\right|_{\partial F}$ uniformly on $\partial F$, whence we get (4.34).

\footnotetext{
${ }^{6}$ In fact, we have shown that $s_{\Omega}$ admits no positive harmonic minorant.
} 
One direction in (4.35) follows by Fatou's lemma:

$$
\int_{M}\left|\nabla s_{\Omega}\right|^{2} \leq \lim _{k \rightarrow \infty} \int_{\mathcal{E}_{k}}\left|\nabla s_{k}\right|^{2}=\lim _{k \rightarrow \infty} \operatorname{cap}\left(F, \mathcal{E}_{k}\right)=\operatorname{cap}(F) .
$$

To prove the other direction (which will be not used in the sequel, though) let us write

$$
\int_{\mathcal{E}_{k} \backslash F}\left|\nabla\left(s_{\Omega}-s_{k}\right)\right|^{2}=\int_{\mathcal{E}_{k} \backslash F}\left|\nabla s_{\Omega}\right|^{2}-2 \int_{\mathcal{E}_{k} \backslash F} \nabla s_{\Omega} \nabla s_{k}+\int_{\mathcal{E}_{k} \backslash F}\left|\nabla s_{k}\right|^{2} .
$$

The last term on the right hand side of $(4.38)$ is equal to $\operatorname{cap}\left(F, \mathcal{E}_{k}\right)$, by (4.37). By the Green formula and (4.34), the middle term transforms into

$$
-\int_{\mathcal{E}_{k} \backslash F} \nabla s_{\Omega} \nabla s_{k}=\int_{\mathcal{E}_{k} \backslash F} s_{k} \Delta s_{\Omega}-\int_{\partial\left(\mathcal{E}_{k} \backslash F\right)} s_{k} \frac{\partial s_{\Omega}}{\partial \nu}=-\int_{\partial F} \frac{\partial s_{\Omega}}{\partial \nu}=-\operatorname{cap}(F) .
$$

Therefore, (4.38) implies

$$
0 \leq \int_{\mathcal{E}_{k} \backslash F}\left|\nabla\left(s_{\Omega}-s_{k}\right)\right|^{2}=\int_{\mathcal{E}_{k} \backslash F}\left|\nabla s_{\Omega}\right|^{2}-2 \operatorname{cap}(F)+\operatorname{cap}\left(F, \mathcal{E}_{k}\right),
$$

whence by letting $k \rightarrow \infty$

$$
\int_{M}\left|\nabla s_{\Omega}\right|^{2} \geq 2 \operatorname{cap}(F)-\lim _{k \rightarrow \infty} \operatorname{cap}\left(F, \mathcal{E}_{k}\right)=\operatorname{cap}(F),
$$

which was to be proved.

\section{Equivalent DEFinitions of RECURREnCE}

Manifold $M$ is said to be non-parabolic if it admits a non-constant positive superharmonic function, and parabolic otherwise. The following theorem provides a number of conditions equivalent to parabolicity. In particular, the parabolicity of $M$ turns out to be equivalent to the recurrence of the Brownian motion $X_{t}$ on $M$.

Theorem 5.1. Let $M$ be a Riemannian manifold. The following properties are equivalent.

(1) Brownian motion on $M$ is transient; i.e. for some open set $U$ and for some point $x \in M$, the process $X_{t}$ eventually leaves $U$ with a positive probability:

$$
\mathbb{P}_{x}\left\{\exists T: \forall t>T \quad X_{t} \notin U\right\}>0 .
$$

(1a) For any precompact set $U \subset M$ and any point $x \in M$, the process $X_{t}$ eventually leaves $U$ with the probability 1 , i.e.

$$
\mathbb{P}_{x}\left\{\exists T: \forall t>T \quad X_{t} \notin U\right\}=1 .
$$

(2) There exists a proper massive set $\Omega$ on $M$.

(2a) The exterior of any compact set on $M$ is $D$-massive.

(3) There exists a non-constant positive superharmonic function on $M$ (=there exists a non-constant bounded subharmonic function on $M)$.

(4) The Green function $G(x, y)$ on $M$ is finite for some/all $x \neq y$.

(5) For some/all $x \in M$,

$$
\int_{1}^{\infty} p(t, x, x) d t<\infty
$$

(6) The capacity of some compact/any precompact open set is positive. 
(7) There exists a non-zero bounded solution on $M$ to the equation

$$
\Delta u-q(x) u=0
$$

for some/any function $q(x) \in C_{0}^{\infty}(M)$, which is non-negative and not identically 0.

(8) If $M$ is a simply connected Riemann surface, then all the above are equivalent to $M$ being of hyperbolic type; i.e. $M$ is conformally equivalent to $\mathbb{H}^{2}$.

Before the proof, let us make some remarks.

- The statement of Theorem 5.1 remains true if $M$ is a manifold with boundary. As we have mentioned before, in this case the heat kernel and the Green function satisfy the Neumann boundary conditions on $\partial M$ and the Brownian motion $X_{t}$ reflects at $\partial M$. A superharmonic function $u$ should satisfy on $\partial M$ the condition $\frac{\partial u}{\partial \nu} \geq 0$, where $\nu$ is the outward normal unit vector field on $\partial M$, and a subharmonic function should satisfy the opposite inequality. Finally, a solution to (5.2) should satisfy the Neumann boundary condition on $\partial M$.

- The essential part of Theorem 5.1 is due to Ahlfors [2], who clarified, for the case of Riemann surfaces, the equivalences $(2) \Leftrightarrow(3) \Leftrightarrow(4)$. His treatment was based in turn on the works of Myrberg [144], Nevanlinna [148] and Ohtsuka [152]. See [167, pp.29-30] for the case of Riemannian manifolds. Equivalence $(2) /(2 a) \Leftrightarrow(4)$ was rediscovered by many authors in various settings: see for example [163, Proposition 23], [111, Lemma 5.2], [88, Theorem 1.1], [122, p.1137].

- The implication $(1) \Rightarrow(4)$ is due to Hunt [98]. Equivalence of (4) and (5) is obvious from the relation (4.5) between the Green function and the heat kernel . A direct proof that (5) is equivalent to (1) can be found in [111, Lemma 3.1].

- In the view of Proposition 4.5, the hypothesis (6) is equivalent to $s_{\Omega} \not \equiv 1$ where $\Omega$ is an exterior of a compact. Equivalence of (4) and $s_{\Omega} \not \equiv 1$ was proved by Ahlfors [2] and Royden [163, Theorem 3]. The idea of using $s_{\Omega}$ for classification of Riemann surfaces is due to Nevanlinna [150]. We adopt here a different approach to the capacity criterion (6) based on the direct relation (4.24) between the Green function and the capacity [127], [71, Proposition 3]. - Equivalence of (7) and (3) was proved in [76, p.2341].

- Equivalence of transience (1) and hyperbolicity (8) is due to Kakutani [105].

- There is some mismatch in the usage of the term "parabolic" here and in the theory of Riemann surfaces. First, any simply connected Riemann surface of elliptic or parabolic type is parabolic in the sense of our definition. However, a simply connected Riemann surface of hyperbolic type is non-parabolic in our sense, which is stated in (8). Second, for a Riemann surface which is not simply connected, its type has nothing to do with parabolicity in our sense, because its type is determined by that of its universal cover.

- We do not apply the adjective "hyperbolic" as an antonym to "parabolic" because there are other generalizations of the notion of hyperbolicity, for example, Gromov's hyperbolicity. 
Proof. We will prove the following sequence of implications:

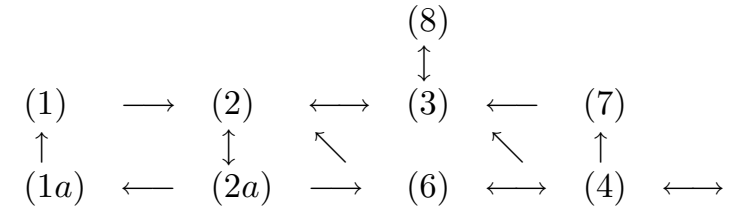

If there is the "some/all" alternative, then we assume the weakest one and prove the strongest one. Not all of the implications above are logically necessary. However, it is useful to be able to independently close different smaller circles of implications.

Another proof of equivalence of (1), (3) and (4) can be found in [130].

(1a) $\Longrightarrow(1)$ Obvious.

(1) $\Longrightarrow(2)$ Let us denote $\Omega=M \backslash \bar{U}$ and consider the function $v:=1-P_{\infty} s_{\Omega}$, which is equal, by Proposition 4.4, to $1-h_{\bar{U}}$, that is to the $\mathbb{P}_{x}$-probability of $X_{t}$ leaving $U$ after some time. By hypothesis, we have $v(x)>0$, for some $x$, whence $P_{\infty} s_{\Omega} \not \equiv 1, s_{\Omega} \neq 1$ and $\Omega$ is massive.

(2a) $\Longrightarrow(2)$ Obvious.

$(\mathbf{2}) \Longrightarrow(\mathbf{2 a})$ Let $\Omega$ be a proper massive set, which exists by hypothesis, and let $\Omega^{\prime}$ be an exterior of some compact. We need to show that $\Omega^{\prime}$ is $D$-massive. Let $U$ be a precompact open set in $M$ which does not intersect $\bar{\Omega}$. Denote $\Omega^{\prime \prime}=M \backslash \bar{U}$. Since $\Omega^{\prime \prime} \supset \Omega$, the set $\Omega^{\prime \prime}$ is massive. By Proposition 4.2, $\Omega^{\prime}$ is massive too because $\Omega^{\prime \prime}$ and $\Omega^{\prime}$ differ by a compact. Finally, since $\Omega^{\prime}$ is exterior of a compact, its massiveness implies its $D$-massiveness, by Corollary 4.6 .

(2a) $\Longrightarrow(\mathbf{1 a})$ We have to prove that, with the $\mathbb{P}_{x}$-probability 1 , the Brownian trajectory $X_{t}$ leaves any precompact set $U$ after some time forever, that is $h_{\bar{U}}(x) \equiv$ 0 . The latter is equivalent, by Proposition 4.4 , to $P_{\infty} s_{\Omega} \equiv 0$ where $\Omega:=M \backslash \bar{U}$. By Proposition 4.5, $P_{\infty} s_{\Omega} \equiv 0$ is equivalent to massiveness of $\Omega$, which we have by hypothesis.

(2a) $\Longrightarrow(\mathbf{6})$ This is part (b) of Corollary 4.6.

(6) $\Longrightarrow(2)$ Let $\operatorname{cap}(U)>0$ for some precompact open set $U$. We may assume that $U$ has smooth boundary. By Corollary 4.6, the set $\Omega:=M \backslash \bar{U}$ is massive.

$(2) \Longrightarrow(3)$ By slightly enlarging $\Omega$, we may assume that $\Omega$ has smooth boundary. Since $\Omega$ is massive and $M \backslash \bar{\Omega}$ is non-empty, $s_{\Omega}$ is a non-trivial bounded superharmonic function on $M$ (see Proposition 4.3(i)).

$(3) \Longrightarrow(2)$ Let $v>0$ be a non-constant superharmonic function on $M$. For any number $c \in(\inf v, \sup v)$, the set $\Omega=\{v<c\}$ is proper and massive because $(c-v)_{+}$is an admissible subharmonic function for $\Omega$.

$(6) \Longleftrightarrow(4)$ Let $U$ be a precompact open set in $M$. For a point $y \in U$, we have, by $(4.24)$,

$$
\inf _{x \in \partial U} G(x, y) \leq \operatorname{cap}(U)^{-1} \leq \sup _{x \in \partial U} G(x, y) .
$$

Therefore, the finiteness of $G$ is equivalent to $\operatorname{cap}(U)>0$.

$(4) \Longrightarrow(3)$ If the Green function $G(x, y)$ is finite, then it is already a positive superharmonic function in $x$ although taking the value $+\infty$ at $x=y$. The truncated function $\min (G(\cdot, y), C)$ (for a positive constant $C$ ) is finite, positive and superharmonic.

$(7) \Longrightarrow(3)$ If $u$ is a bounded non-zero solution to $\Delta u-q u=0$ with some function $q \in C_{0}^{\infty}, q \not \equiv 0$, then either $u_{+}$or $u_{-}$is not identically zero. Assume that $u_{+} \not \equiv 0$. We claim that $u_{+}$is a non-constant bounded subharmonic function. The function 
$u_{+}$is subharmonic in $\Omega:=\{u>0\}$ just because $\Delta u_{+}=q u_{+} \geq 0$. Since $u_{+}$is extended by 0 outside $\Omega$, function $u_{+}$is subharmonic in $M$. Finally, $u_{+}$is bounded and non-constant because if $u_{+} \equiv c$ then $u \equiv c$, which is only possible if $q \equiv 0$ or $c=0$.

(3) $\Longleftrightarrow(8)$ Conformal mapping in dimension 2 preserves superharmonic functions. Since $\mathbb{H}^{2}$ possesses a non-constant positive superharmonic function whereas $\mathbb{R}^{2}$ does not, hyperbolicity of $M$ is equivalent to the presence of a non-constant positive superharmonic function.

$(4) \Longleftrightarrow(5)$ Since

$$
G(x, y)=\frac{1}{2} \int_{0}^{\infty} p(t, x, y) d t
$$

the condition $G(x, y)<\infty$ implies, for those $x, y$,

$$
\int^{\infty} p(t, x, y) d t<\infty
$$

By the local parabolic Harnack inequality, for any pair $x^{\prime}, y^{\prime} \in M$, we have, for all $t$ large enough,

$$
\frac{p\left(t, x^{\prime}, y^{\prime}\right)}{p(t, x, y)}<\mathrm{const}
$$

(see for details [45, Theorem 10]). In particular, (5.4) holds for all pairs $x, y$, whence (5.1) follows. Conversely, if (5.1) holds for some $x \in M$, then (5.4) is true by (5.5) for all $x, y \in M$. Since, for $x \neq y$, we have $p(t, x, y) \rightarrow 0$ as $t \rightarrow 0$, the integral in (5.3) converges also at 0 , and $G(x, y)<\infty$.

$(4) \Longrightarrow(7)$ Let $\left\{\mathcal{E}_{k}\right\}$ be an exhaustion sequence and $G_{k}$ be the Green kernel in $\mathcal{E}_{k}$. Denote by $u_{k}$ and $v_{k}$ the solutions of the following Dirichlet problems in $\mathcal{E}_{k}$

$$
\left\{\begin{array} { l } 
{ \Delta u _ { k } - q u _ { k } = 0 } \\
{ u _ { k } | _ { \partial \mathcal { E } _ { k } } = 1 }
\end{array} \text { in } \mathcal { E } _ { k } \quad \text { and } \quad \left\{\begin{array}{l}
\Delta v_{k}=-q \text { in } \mathcal{E}_{k} \\
\left.v_{k}\right|_{\partial \mathcal{E}_{k}}=0
\end{array}\right.\right.
$$

The sequence $\left\{u_{k}\right\}$ is decreasing and converges to a function $u$, which is a bounded solution to $\Delta u-q u=0$. Since $0 \leq u_{k} \leq 1$, we have also $0 \leq u \leq 1$, and it will suffice to show that $\sup u>0$.

The function $v_{k}$ is given by

$$
v_{k}=\int_{\mathcal{E}_{k}} G_{k}(\cdot, y) q(y) d \mu(y),
$$

whence we see that $\left\{v_{k}\right\}$ is increasing and converges to

$$
v:=\int_{M} G(\cdot, y) q(y) d \mu(y) .
$$

The function $u_{k}+v_{k}$ is superharmonic because $\Delta\left(u_{k}+v_{k}\right)=q u_{k}-q \leq 0$. Since $u_{k}+\left.v_{k}\right|_{\partial \mathcal{E}_{k}}=1$, the minimum principle says that $u_{k}+v_{k} \geq 1$ and, thus, $u_{k} \geq 1-v_{k}$ in $\mathcal{E}_{k}$ (see Figure 16).

Therefore, $u \geq 1-v$. We claim that, in fact, $\inf v=0$, which would imply $\sup u>0$. By the construction, $v$ is the smallest non-negative solution to $\Delta v=-q$ $\operatorname{in} M$. If we assume $\inf v>0$, then the function $v-\inf v$ is also a non-negative solution to this equation, which contradicts the minimality of $v$. Thus, we obtain $\inf v=0$ and $\sup u>0$.

Let us show some applications of Theorem 5.1. 


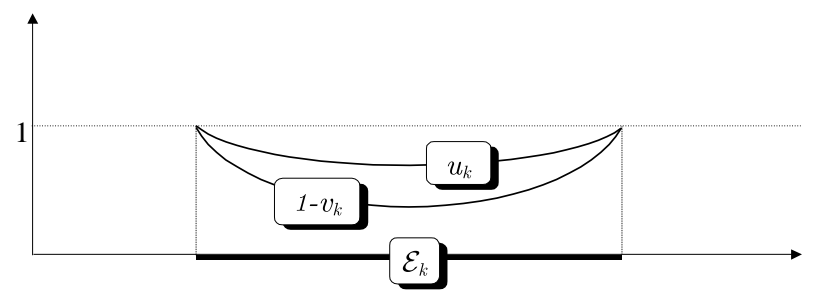

FiguRE 16. Functions $u_{k}$ and $1-v_{k}$

Corollary 5.2. Let $U$ be a non-empty precompact open subset of $M$. The following statements are equivalent.

(i) $M$ is non-parabolic.

(ii) The set $\Omega:=M \backslash \bar{U}$ is massive.

(iii) $\operatorname{cap}(U)=0$.

This is just another way to state the parts (2)/(2a) and (6) of Theorem 5.1. Let us emphasize that it suffices to test only one set $U$ to decide whether $M$ is parabolic or not.

One says that manifolds $M_{1}$ and $M_{2}$ are quasi-isometric if there exists a quasiisometry ${ }^{7} F: M_{1} \rightarrow M_{2}$, that is, a diffeomorphic map from $M_{1}$ onto $M_{2}$ such that, for all $x, y \in M_{1}$ and some constant $C>0$,

$$
C^{-1} \operatorname{dist}_{M_{2}}(F(x), F(y)) \leq \operatorname{dist}_{M_{1}}(x, y) \leq C \operatorname{dist}_{M_{2}}(F(x), F(y)) .
$$

Corollary 5.3. Let $M$ and $N$ be two Riemannian manifolds. If the exteriors of some compacts in $M$ and $N$ are quasi-isometric, then $M$ and $N$ are parabolic or not simultaneously. In particular, if $M$ and $N$ are quasi-isometric, then $M$ and $N$ are parabolic or not simultaneously.

Proof. Let $K$ and $K^{\prime}$ be the compacts in $M$ and $N$ whose exteriors are quasiisometric, and let $U$ be a precompact neighborhood of $K$ in $M$. Obviously, there is a precompact open set $U^{\prime} \subset N$ such that $M \backslash \bar{U}$ and $N \backslash \overline{U^{\prime}}$ are quasi-isometric. As follows from the definition (4.12) of capacity, the value of the capacity $\operatorname{cap}_{M}(U)$ depends only on the intrinsic geometry of $M \backslash \bar{U}$. The same applies to $\operatorname{cap}_{N}\left(U^{\prime}\right)$. The capacity changes under a quasi-isometry at most by a constant factor. Therefore, $\operatorname{cap}_{M}(U)=0$ if and only if $\operatorname{cap}_{N}\left(U^{\prime}\right)=0$. Hence, by Corollary 5.2, $M$ and $N$ are parabolic or not simultaneously.

It is possible to prove that the $D$-massiveness is stable under a quasi-isometry (see Theorem 14.2 below). This means also that the massiveness of an exterior of a compact is a quasi-isometry invariant, too. However, in general, massiveness is not invariant under a quasi-isometry as follows from [129, Section 8] and [77, Corollary 1]. See also [107] for stability of parabolicity under rough isometries and [94] for generalizations to $p$-harmonic theory.

Corollary 5.4. (Khas'minskii [111]) If there is a superharmonic function $v$ outside a compact $K$ such that $v(x) \rightarrow+\infty$ as $x \rightarrow \infty$, then $M$ is parabolic.

Proof. Let us enlarge $K$ so that $\partial K$ is smooth and $v$ is non-negative on $\Omega:=M \backslash K$. Consider a sequence $\left\{b_{k}\right\}$ of solutions to the Dirichlet problem (4.27) in $\mathcal{E}_{k} \backslash K$. By

\footnotetext{
${ }^{7}$ Another related notion is a rough isometry - see [107].
} 
Theorem 5.1(2a), the parabolicity of $M$ will follow from the non-massiveness of $\Omega$, which in turn is equivalent to

$$
b_{\Omega}=\lim _{k \rightarrow \infty} b_{k}=0
$$

To show that, fix any $C>>1$ and observe that $v \rightarrow \infty$ implies, for all $k$ big enough,

$$
\inf _{\partial \mathcal{E}_{k}} v \geq C .
$$

Therefore, by the maximum principle in $\mathcal{E}_{k} \backslash K$, we have $v \geq C b_{k}$, whence $C^{-1} v \geq$ $b_{\Omega}$. By letting $C \rightarrow \infty$, we obtain $b_{\Omega}=0$.

Similarly if, in an open set $\Omega$, there exists a superharmonic function $v$ such that $v(x) \rightarrow+\infty$ as $x \rightarrow \infty$, then $\Omega$ is non-massive.

The converse to Corollary 5.4 is also true, and we present it without proof.

Theorem 5.5. (Nakai [146]) If $M$ is parabolic, then there exists a harmonic function $v(x)$ outside a compact such that $v(x) \rightarrow+\infty$ as $x \rightarrow \infty$.

The following statement is a consequence of Theorem 5.1 and the explicit formula (4.23) for the capacity of a ball on a model manifold.

Corollary 5.6. (=Proposition 3.1) A model manifold $M_{\sigma}$ is parabolic if and only if

$$
\int^{\infty} \frac{d \rho}{S(\rho)}=\infty
$$

In the following theorem, we have collected some other criteria of parabolicity, which we present without proof. Given a precompact open set $U \subset M$, we denote by $D_{U}(M)$ a Banach space which is the completion of $C_{0}^{\infty}(M)$ with respect to the following norm

$$
\|f\|_{D_{U}}:=\int_{U}|f| d \mu+\left(\int_{M}|\nabla f|^{2} d \mu\right)^{\frac{1}{2}} .
$$

Theorem 5.7. Each of the following properties is equivalent to parabolicity of $M$.

(a) (Beurling-Deny) For some precompact open set $U \subset M$,

$$
1 \in D_{U}(M) .
$$

(b) (Royden [163, Theorem 4, p.66], Lyons-Sullivan [132, p.312]) For any smooth vector field $v$ on $M$ such that $|v| \in L^{2}(M, \mu)$ and $\operatorname{div} v \in L^{1}(M, \mu)$, we have

$$
\int_{M} \operatorname{div} v d \mu=0 .
$$

(c) ([73, Proposition 2]) For any bounded function $u \in C^{2}(M)$ such that $\Delta u \in$ $L^{1}(M, \mu)$, we have

$$
\int_{M} \Delta u d \mu=0
$$

The condition (5.6) is close to the fact that cap $(U)=0$. Roughly speaking, in the definition of the capacity, the class of test functions can be extended to $D_{U}(M)$. Therefore, if $1 \in D_{U} M$, then cap $(U)=0$. The converse can be proved too (see [ 47 , pp.181-182], [163, p.67], [5, p.46]).

The identities (5.7) and (5.8) mean that there is no boundary term in the Green formula when integrating over the entire $M$. This reflects the fact that a parabolic 
manifold has in some sense no boundary at infinity, which causes the Brownian trajectory to enter any bounded region again and again.

\section{EQUiVALENT DEFINITIONS OF STOCHASTIC COMPLETENESS}

As we have seen, the notion of massiveness plays an important role when dealing with the recurrence of the Brownian motion. Some extension of this notion - $\lambda$ massiveness - will be useful for treatment of stochastic completeness. For any $\lambda>0$, we say that $u$ is $\lambda$-harmonic function if it satisfies the equation

$$
\Delta u-\lambda u=0 .
$$

Similarly to sub- and superharmonic functions (see Section 4.1), one defines continuous $\lambda$-sub- and $\lambda$-superharmonic functions.

Definition 6.1. Given an open set $\Omega \subset M$, we say that a function $v$ is an admissible $\lambda$-subharmonic function for $\Omega$ if it is a non-negative bounded $\lambda$-subharmonic function on $M$ such that $v=0$ in $M \backslash \Omega$ and $\sup _{\Omega} v>0$. An open set $\Omega$ is called $\lambda$-massive if there is at least one admissible $\lambda$-subharmonic function for $\Omega$.

The empty set is never $\lambda$-massive. However, the whole manifold is not necessarily $\lambda$-massive because the function $v \equiv 1$ is not $\lambda$-subharmonic unlike the case $\lambda=0$.

Similarly to Proposition 4.2, we have

Proposition 6.1. The $\lambda$-massiveness is preserved by enlarging $\Omega$ and by reducing it by a compact.

Remark. Unlike Proposition 4.2, we do not have to assume in the second statement that $\Omega$ is proper.

The following theorem provides various conditions equivalent to the explosion property.

Theorem 6.2. The following properties are equivalent.

(1) The manifold $M$ is stochastically incomplete; that is, for some $(x, t) \in M \times$ $(0, \infty)$,

$$
\int_{M} p(t, x, y) d \mu(y)<1 .
$$

(1a) For all $(x, t) \in M \times(0, \infty)$, we have (6.1).

(2) $M$ is $\lambda$-massive.

(3) For some/all $\lambda>0$, there is a non-zero bounded $\lambda$-harmonic function on $M$.

(4) For some/any $T \in(0, \infty)$, the Cauchy problem

$$
\left\{\begin{array}{l}
\frac{\partial u}{\partial t}=\frac{1}{2} \Delta u \\
\left.u\right|_{t=0^{+}}=0
\end{array}\right.
$$

has a non-zero bounded solution in $M \times(0, T)$ (the initial data is understood in $\left.L_{l o c}^{1}(M, \mu)\right)$.

Remark. The part (2) looks simpler than its analogue in Section 5 - Theorem 5.1(2)-(2a). Nonetheless, it is equivalent to either of the following assertions:

(2a) For some $\lambda>0$, there is a $\lambda$-massive set.

(2b) For any $\lambda>0$, the exterior of any compact set is $\lambda$-massive. 
Indeed, the existence of a $\lambda$-massive set implies that $M$ is $\lambda$-massive, by the first part of Proposition 6.1. If $M$ is $\lambda$-massive then the exterior of any compact is $\lambda$-massive, by the second part of Proposition 6.1.

Theorem 6.2 is largely due to Khas'minskii [111, Lemmas 4.1, 5.1 ]. The criterion (3) goes back to Feller [59] in the case of one-dimensional diffusions. The proofs of different parts of this theorem in various settings can also be found in [41], [75], [76].

Proof. We will prove the following chain of implications:

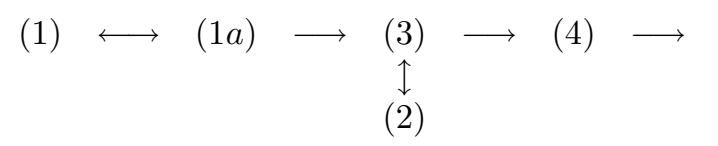

$(\mathbf{1}) \Leftrightarrow \mathbf{( 1 a )}$ The fact that (1a) implies (1) is obvious. Let us assume (1) and prove (1a). By the semi-group property, we have, for all $s \in(0, t)$,

$$
P_{t} 1=P_{t-s} P_{s} 1 \leq P_{t-s} 1 \leq 1 \text {. }
$$

Since we know that $P_{t} 1(x)=1$ holds for some $x \in M$, we conclude that, for this $x$, all inequalities in (6.3) become equalities. In particular, we have

$$
P_{t-s}\left(P_{s} 1\right)(x)=1
$$

which is only possible if

$$
P_{s} 1 \equiv 1
$$

We are left to extend (6.4) to $s \geq t$. Let first $s<2 t$. Then $s / 2<t$ and we obtain, by the semi-group property,

$$
P_{s} 1=P_{s / 2}\left(P_{s / 2} 1\right)=P_{s / 2} 1=1
$$

that is, (6.4) holds also for $s \in(0,2 t)$. By induction, we prove (6.4) for $s \in\left(0,2^{k} t\right)$, whence it follows for all $s>0$.

(1a) $\Rightarrow(\mathbf{3})$ Given $\lambda>0$, let us set $u(x, t):=P_{t} 1<1$ and

$$
w(x):=\int_{0}^{\infty} e^{-\lambda t} u(x, t) d t .
$$

It is easy to verify that

$$
\begin{aligned}
\frac{1}{2} \Delta w & =\int_{0}^{\infty} e^{-\lambda t} \frac{1}{2} \Delta u d t=\int_{0}^{\infty} e^{-\lambda t} \frac{\partial u}{\partial t} d t \\
& =\left.u e^{-\lambda t}\right|_{0} ^{\infty}+\lambda \int_{0}^{\infty} e^{-\lambda t} u d t=-1+\lambda w
\end{aligned}
$$

and

$$
0<w<\int_{0}^{\infty} e^{-\lambda t} d t=\lambda^{-1}
$$

Therefore, the function $v:=1-\lambda w$ satisfies the equation $\frac{1}{2} \Delta v-\lambda v=0$ and $0<v<1$. We are left to rename $2 \lambda$ to $\lambda$.

$(3) \Rightarrow(4)$ Let $v(x)$ be a non-zero bounded $\lambda$-harmonic function. Clearly, the function

$$
u(x, t)=v(x) e^{\frac{1}{2} \lambda t}
$$


solves the Cauchy problem

$$
\left\{\begin{array}{l}
\frac{\partial u}{\partial t}=\frac{1}{2} \Delta u \\
\left.u\right|_{t=0^{+}}=v(x)
\end{array}\right.
$$

On the other hand, there is another solution to (6.5), namely, $w=P_{t} v$. For any $t>0$, we have

$$
\sup |w|(\cdot, t) \leq \sup |v| \cdot P_{t} 1 \leq \sup |v|,
$$

whereas $v \not \equiv 0$ implies

$$
\sup |u|(\cdot, t)=e^{\frac{1}{2} \lambda t} \sup |v|>\sup |v| .
$$

Therefore, the functions $u(\cdot, t)$ and $w(\cdot, t)$ are different, for any $t>0$. At the same time, both are bounded on $M \times(0, T)$, whence we conclude that $u-w$ is a non-zero bounded solution to $(6.2)$ on $M \times(0, T)$.

$(4) \Rightarrow(1)$ Let $u(x, t)$ be a non-zero bounded solution to $(6.2)$, for some $T>0$. We can assume that $\sup u>0$ and $\sup |u|<1$ so that the function $w:=1-u$ is positive and $\inf w<1$. Since the function $w$ is a solution to the Cauchy problem

$$
\left\{\begin{array}{l}
\frac{\partial w}{\partial t}=\frac{1}{2} \Delta w \\
\left.w\right|_{t=0^{+}}=1
\end{array}\right.
$$

and $P_{t} 1$ is the minimal positive solution to (6.6), we conclude that $P_{t} 1 \leq w$. Therefore, for some $x \in M$ and $t \in(0, T)$,

$$
P_{t} 1=\int_{M} p(t, x, y) d \mu(y)<1,
$$

and $M$ is stochastically incomplete.

$(3) \Rightarrow(2)$ If $v$ is a non-zero bounded $\lambda$-harmonic function, then one of the functions $v_{+}, v_{-}$must be non-zero; let it be $v_{+}$. Clearly, $v_{+}$is $\lambda$-harmonic in $\{v>0\}$ which implies that $v_{+}$is $\lambda$-subharmonic in $M$, and $M$ is $\lambda$-massive.

(2) $\Rightarrow(3)$ Let $M$ be $\lambda$-massive and let $w$ be an admissible $\lambda$-subharmonic function for $M$. We will construct a non-zero bounded $\lambda$-harmonic function on $M$ as the limit of solutions to the following Dirichlet problems

$$
\left\{\begin{array}{l}
\Delta v_{k}-\lambda v_{k}=0 \text { in } \mathcal{E}_{k}, \\
\left.v_{k}\right|_{\partial \mathcal{E}_{k}}=1
\end{array}\right.
$$

where $\left\{\mathcal{E}_{k}\right\}$ is an exhaustion sequence. We have $0 \leq v_{k} \leq 1$, and the sequence $\left\{v_{k}\right\}$ is decreasing and converges to a bounded solution $v$. Let us verify that $v \not \equiv 0$. We may assume from the beginning that $\sup w=1$. Then we have, by the maximum principle, $v_{k} \geq w$ and thus $v \geq w$ which implies $v \not \equiv 0$.

Corollary 6.3. (Khas'minskii [111]) The stochastic completeness of $M$ is equivalent to the uniqueness for the Cauchy problem (6.2) on $M \times(0, \infty)$ in the class of bounded solutions.

Proof. It suffices to show that if $M$ is stochastically incomplete then there is a bounded non-zero solution to $(6.2)$ on $M \times(0, \infty)$. The function $u(x, t)=1-P_{t} 1(x)$ is such a solution (note that in the proof of Theorem 6.2, we have constructed such a solution on a finite time interval). Indeed, it is obviously bounded and is non-zero because $P_{t} 1(x) \not \equiv 1$.

Corollary 6.4. If $M$ is parabolic, then $M$ is stochastically complete.

Indeed, $\lambda$-massiveness implies massiveness. 
Corollary 6.5. If the exteriors of some compacts in $M_{1}$ and $M_{2}$ are isometric, then $M_{1}, M_{2}$ are stochastically complete or not simultaneously.

This follows from the fact that $\lambda$-massiveness is preserved by subtracting a compact (Proposition 6.1).

Corollary 6.6. ([111], [184, Proposition 1]) If, for some $\lambda>0$, there is a $\lambda$ superharmonic function $v$ outside a compact set in $M$ such that $v(x) \rightarrow+\infty$ as $x \rightarrow \infty$, then $M$ is stochastically complete.

The proof is similar to that of Corollary 5.4.

Corollary 6.7. ([75]) If, for some point $x \in M$ and a precompact open set $U \ni x$,

$$
\int_{M \backslash U} G(x, y) d \mu(y)<\infty,
$$

then $M$ is not stochastically complete.

Proof. Assume on the contrary that $M$ is stochastically complete. Then, for all $x \in M, t>0$, we have $P_{t} 1=1$, whence

$$
\begin{aligned}
\int_{M} G(x, y) d \mu(y) & =\frac{1}{2} \int_{M} \int_{0}^{\infty} p(t, x, y) d t d \mu(y) \\
& =\frac{1}{2} \int_{0}^{\infty} \int_{M} p(t, x, y) d \mu(y) d t \\
& =\frac{1}{2} \int_{0}^{\infty} d t=\infty .
\end{aligned}
$$

By (4.6), the singularity of the Green function is summable, whence

$$
\int_{U} G(x, y) d \mu(y)<\infty
$$

contradicting (6.7) and (6.8).

Corollary 6.8. (=Proposition 3.2) A model manifold $M_{\sigma}$ is stochastically complete if and only if

$$
\int^{\infty} \frac{V(r) d r}{S(r)}=\infty
$$

Proof. The Green function on $M_{\sigma}$ is

$$
G(o, x)=\int_{\rho}^{\infty} \frac{d r}{S(r)}
$$

where $x=(\rho, \theta)$. Therefore, the sufficient condition (6.7) for stochastic incompleteness acquires the form

$$
\int^{\infty} S(\rho) d \rho \int_{\rho}^{\infty} \frac{d r}{S(r)}<\infty
$$

or, after interchanging the order of the integrals,

$$
\int^{\infty} \frac{V(r)}{S(r)} d r<\infty
$$


Thus, (6.10) implies stochastic incompleteness.

Let us show that conversely (6.9) implies the stochastic completeness of $M$. If

$$
\int^{\infty} \frac{d r}{S(r)}=\infty
$$

then $M_{\sigma}$ is parabolic and thus stochastically complete. Assume in the sequel that

$$
\int^{\infty} \frac{d r}{S(r)}<\infty
$$

If a function $v$ on $M_{\sigma}$ depends only on the polar radius $\rho$, then the equation $\Delta v-v=0$ defining 1-harmonic functions amounts to

$$
v^{\prime \prime}+\frac{S^{\prime}}{S} v^{\prime}-v=0
$$

Let $v$ solve $(6.11)$ on $[1, \infty)$ with the initial values $v(1)=1$ and $v^{\prime}(1)=0$. The function $v(\rho)$ is monotone increasing because equation (6.11), after multiplying by $S v$ and integrating from 1 to $\rho$, amounts to

$$
S v v^{\prime}(\rho)=\int_{1}^{\rho} S\left(v^{2}+v^{2}\right) d r \geq 0 .
$$

Equation (6.11) can be transformed also into the integral equation

$$
v(\rho)=1+\int_{1}^{\rho} \frac{d r}{S(r)} \int_{1}^{r} S(\xi) v(\xi) d \xi
$$

which implies, together with $v \geq 1$, that

$$
v(\rho) \geq \int_{1}^{\rho} \frac{d r}{S(r)} \int_{1}^{r} S(\xi) d \xi=\int_{1}^{\rho} \frac{(V(r)-V(1)) d r}{S(r)} \geq \int_{1}^{\rho} \frac{V(r) d r}{S(r)}-\text { const. }
$$

Therefore, (6.9) implies that $v(\rho) \rightarrow \infty$ as $\rho \rightarrow \infty$, and $M$ is stochastically complete by Corollary 6.6.

\section{Parabolicity and volume Growth}

As follows from Theorem 5.1, in order to prove that a manifold is parabolic, it suffices to show that capacity of some precompact open set is 0 . This motivates us to consider the following estimates of capacity.

\subsection{Upper bounds of capacity.}

Theorem 7.1. Assume that a ball $B(x, R)$ on a Riemannian manifold $M$ is precompact. Then, for any $0<r<R$, the following estimates of capacity are satisfied:

$$
\operatorname{cap}(B(x, r), B(x, R))^{-1} \geq \frac{1}{2} \int_{r}^{R} \frac{(\rho-r) d \rho}{V(x, \rho)-V(x, r)}
$$

and

$$
\operatorname{cap}(B(x, r), B(x, R))^{-1} \geq \int_{r}^{R} \frac{d \rho}{V^{\prime}(x, \rho)} .
$$

Corollary 7.2. Assume that $M$ is geodesically complete. Then, for any $r>0$ and $x \in M$,

$$
\operatorname{cap}(B(x, r))^{-1} \geq \frac{1}{2} \int_{r}^{\infty} \frac{(\rho-r) d \rho}{V(x, \rho)-V(x, r)}
$$


and

$$
\operatorname{cap}(B(x, r))^{-1} \geq \int_{r}^{\infty} \frac{d \rho}{V^{\prime}(x, \rho)} .
$$

Remark. The geodesic completeness of $M$ is required here to ensure that all balls are precompact sets.

The estimate (7.2) is contained in [134, section 2.2.2, Lemma 1], in the context of a general notion of capacities in $\mathbb{R}^{d}$. For Riemannian manifolds, it was proved in [71, Theorem 1]. The estimate (7.1) was proved by Sturm [174]. A very similar inequality appears also in [153].

Inequality (7.2) is sharp in the sense that it becomes an equality if $M$ is a model manifold and $x$ is its pole. Inequality (7.1) is sharp in the sense that the factor $\frac{1}{2}$ cannot be increased (see [174, p.77]).

The estimates of capacity can be generalized in the following way. Let $v(x)$ be an exhaustion function on $M$, i.e. a continuous function on $M$ such that all level sets $\{x \in M: v(x)<r\}$ are precompact. The latter is equivalent to

$$
v(x) \rightarrow+\infty \text { as } x \rightarrow \infty,
$$

where $x \rightarrow \infty$ means that $x$ is leaving any compact.

Assume that $v(x)$ is locally Lipschitz, and let $\Gamma(x)$ be a continuous function on $M$ satisfying the inequality

$$
|\nabla v(x)|^{2} \leq \Gamma(x)
$$

Let us set $B_{r}=\{x \in M: v(x)<r\}$ and

$$
W(r):=\int_{B_{r}} \Gamma(x) d \mu,
$$

which is an analogue of the volume function. Then, for all $r<R$,

$$
\operatorname{cap}\left(B_{r}, B_{R}\right)^{-1} \geq \frac{1}{2} \int_{r}^{R} \frac{(\rho-r) d \rho}{W(\rho)-W(r)}
$$

and

$$
\operatorname{cap}\left(B_{r}, B_{R}\right)^{-1} \geq \int_{r}^{R} \frac{d \rho}{W^{\prime}(\rho)} .
$$

If $M$ is geodesically complete, then the distance function is an exhaustion function so that we may take $v(\cdot)=\operatorname{dist}(x, \cdot)$. By letting $\Gamma \equiv 1$, we obtain (7.1) and (7.2) from (7.4) and (7.5), respectively.

To get another example, let us set $\Gamma=|\nabla v|^{2}$ assuming that $v$ is smooth enough. By the co-area formula, we have

$$
W^{\prime}(\rho)=\int_{\partial B_{\rho}}|\nabla v| d \mu^{\prime}=\operatorname{flux}_{\partial B_{\rho}} v
$$

for almost all $\rho$, and (7.5) yields

$$
\operatorname{cap}\left(B_{r}, B_{R}\right)^{-1} \geq \int_{r}^{R} \frac{d \rho}{\operatorname{flux}_{\partial B_{\rho}} v} .
$$

The estimate (7.6) was obtained in [71, Lemma 1].

Inequalities (7.4) and (7.5) can be proved in the same way as (7.1) and (7.2) below. 
Proof of inequality (7.1). By the definition (4.12) of capacity, we have

$$
\operatorname{cap}(B(x, r), B(x, R))=\inf _{u} \int_{B(x, R)}|\nabla u|^{2} d \mu
$$

where inf is taken over all test functions $u \in \mathcal{L}(B(x, r), B(x, R))$. To prove (7.1), it suffices to produce, for any $\varepsilon>0$, a test function $u$ such that

$$
\int_{B(x, R)}|\nabla u|^{2} d \mu \leq 2\left(\int_{r}^{R} \frac{s-r}{V(s)-V(r)+\varepsilon} d s\right)^{-1}
$$

where we denote for simplicity $V(s):=V(x, s)$.

Let $f$ be a Lipschitz function on $[0, \infty)$ such that

$$
\left.f\right|_{[0, r)}=1 \quad \text { and }\left.\quad f\right|_{[R, \infty)}=0 .
$$

Let us set $u:=f(\rho(\cdot))$ where $\rho(\cdot):=\operatorname{dist}(x, \cdot)$. Since $|\nabla \rho| \leq 1$, we have

$$
|\nabla u|=\left|f^{\prime}(\rho) \nabla \rho\right| \leq\left|f^{\prime}(\rho)\right|
$$

and

$$
\int_{B(x, R)}|\nabla u|^{2} d \mu \leq \int_{B(x, R)}\left|f^{\prime}(\rho)\right|^{2} d \mu=\int_{r}^{R}\left|f^{\prime}(\rho)\right|^{2} d V(\rho) .
$$

Let us choose $f$ to satisfy on $[r, R]$ the equation

$$
f^{\prime}(\rho)=-a \frac{\rho-r}{V(\rho)-V(r)+\varepsilon}
$$

and the boundary conditions (7.8), whence

$$
f(\rho)=a \int_{\rho}^{R} \frac{s-r}{V(s)-V(r)+\varepsilon} d s
$$

where

$$
a=\left(\int_{r}^{R} \frac{s-r}{V(s)-V(r)+\varepsilon} d s\right)^{-1} .
$$

Thus, by (7.9) and (7.10),

$$
\begin{aligned}
\int_{B(x, R)}|\nabla u|^{2} d \mu & \leq a^{2} \int_{r}^{R}\left(\frac{\rho-r}{V(\rho)-V(r)+\varepsilon}\right)^{2} d V(\rho) \\
& =-a^{2} \int_{r}^{R}(\rho-r)^{2} d\left(\frac{1}{V(\rho)-V(r)+\varepsilon}\right) \\
& =-\left.\frac{a^{2}(\rho-r)^{2}}{V(\rho)-V(r)+\varepsilon}\right|_{r} ^{R}+2 a^{2} \int_{r}^{R} \frac{\rho-r}{V(\rho)-V(r)+\varepsilon} d \rho \\
& \leq 2 a^{2} a^{-1}=2 a,
\end{aligned}
$$

whence (7.7) follows. 
Proof of inequality (7.2). It follows the same lines as above but with a different function $f$. Let us replace (7.10) by

$$
f^{\prime}(\rho)=-\frac{a}{V^{\prime}(\rho)+\varepsilon},
$$

whence

$$
f(\rho)=a \int_{\rho}^{R} \frac{d s}{V^{\prime}(s)+\varepsilon},
$$

and

$$
a=\left(\int_{r}^{R} \frac{d s}{V^{\prime}(s)+\varepsilon}\right)^{-1} .
$$

Then, by (7.9) and (7.11),

$$
\int_{B(x, R)}|\nabla u|^{2} \leq a^{2} \int_{r}^{R} \frac{V^{\prime}(\rho) d \rho}{\left(V^{\prime}(\rho)+\varepsilon\right)^{2}} \leq a^{2} \int_{r}^{R} \frac{d \rho}{V^{\prime}(\rho)+\varepsilon}=a,
$$

whence (7.2) follows as $\varepsilon \downarrow 0$.

7.2. Sufficient conditions for parabolicity. The main result of this section is the following theorem.

Theorem 7.3. Let $M$ be geodesically complete. If, for some point $x \in M$,

$$
\int^{\infty} \frac{\rho d \rho}{V(x, \rho)}=\infty
$$

then $M$ is parabolic.

This theorem was proved by Karp [108], Varopoulos [184] and by the author [70], [71].

Example 7.1. The hypothesis (7.12) is satisfied if, for example,

$$
V(x, \rho) \leq C \rho^{2}
$$

or

$$
V(x, \rho) \leq C \rho^{2} \log \rho,
$$

for some $x$ and all $\rho$ large enough.

Proof. By Corollary 7.2 and by (7.12), we have

$$
\operatorname{cap}(B(x, r)) \leq 2\left(\int_{r}^{\infty} \frac{(\rho-r) d \rho}{V(x, \rho)-V(x, r)}\right)^{-1}=0,
$$

for any ball $B(x, r)$. Therefore, $M$ is parabolic by Theorem 5.1(6).

Corollary 7.4. (Cheng - Yau [28]) Let $M$ be geodesically complete. If, for some point $x \in M$ and for a sequence $R_{k} \rightarrow \infty$,

$$
V\left(x, R_{k}\right) \leq \text { const } R_{k}^{2},
$$

then $M$ is parabolic. 
Proof. Indeed, condition (7.13) implies that

$$
\begin{aligned}
\int^{\infty} \frac{\rho d \rho}{V(x, \rho)} & \geq \sum_{k=2}^{\infty} \int_{R_{k-1}}^{R_{k}} \frac{\rho d \rho}{V(x, \rho)} \\
& \geq \operatorname{const} \sum_{k=2}^{\infty} \frac{1}{R_{k}^{2}} \int_{R_{k-1}}^{R_{k}} \rho d \rho \\
& =\text { const } \sum_{k=2}^{\infty} \frac{R_{k}^{2}-R_{k-1}^{2}}{R_{k}^{2}}=\infty,
\end{aligned}
$$

where divergence of the series (7.14) follows from the fact that $\prod_{k=2}^{\infty} \frac{R_{k-1}^{2}}{R_{k}^{2}}=0$. The rest follows by Theorem 7.3.

Denote $S(x, \rho)=\mu^{\prime}(\partial B(x, \rho))$; that is, $S(x, \rho)$ is the boundary area of the geodesic sphere $\partial B(x, \rho)$. Observe that $S(x, \rho)=V^{\prime}(x, \rho)$, for almost all $\rho$. By using the capacity estimate (7.3), we obtain the following result.

Theorem 7.5. Let $M$ be geodesically complete and, for some $x \in M$,

$$
\int^{\infty} \frac{d \rho}{S(x, \rho)}=\infty
$$

Then $M$ is parabolic.

Theorem 7.5 was proved by Ahlfors [1] and Nevanlinna [149] for Riemann surfaces, by using the conformal mapping argument. For Riemannian manifolds, it was proved by Lyons and Sullivan [132] and by the author [70], [71].

For a model manifold, (7.15) is also a necessary condition for parabolicity, by Proposition 3.1. We will see in the examples below that, in general, (7.15) is not necessary for parabolicity. However, it is possible to modify (7.15) to make it a necessary condition, too. Given an exhaustion function $v$, let us denote $B_{r}=$ $\{x \in M: v(x)<r\}$. If $v$ is smooth, then the set $\partial B_{r}=\{x: v(x)=r\}$ is a smooth hypersurface for almost all $r$.

Theorem 7.6. A manifold $M$ is parabolic if and only if there is a smooth exhaustion function $v$ on $M$ such that

$$
\int^{\infty} \frac{d r}{\operatorname{flux}_{\partial B_{r}} v}=\infty
$$

For example, if $v(\cdot)=\operatorname{dist}(x, \cdot)$ then $\operatorname{flux}_{\partial B_{r}} v \leq S(x, r)$ and Theorem 7.5 follows from Theorem 7.6.

Proof. If (7.16) holds then, by (7.6), $\operatorname{cap}\left(B_{r}\right)=0$ and $M$ is parabolic, by Theorem 5.1(6). Note that in contrast to Theorem 7.5, we do not need here that $M$ is geodesically complete because the sets $B_{r}$ are automatically precompact, by the definition of an exhaustion function.

Assume now that $M$ is parabolic. Then there is an exhaustion sequence of precompact open sets $\left\{\mathcal{E}_{k}\right\}$ such that

$$
\operatorname{cap}\left(\mathcal{E}_{k}, \mathcal{E}_{k+1}\right) \leq 1 .
$$




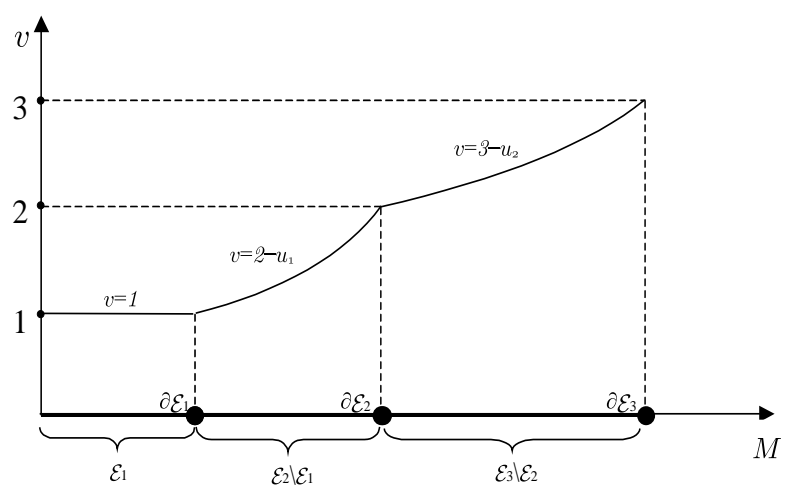

FIGURE 17. Function $v$

Indeed, if $\left\{\mathcal{E}_{k}\right\}$ is some exhaustion sequence, then, for any $k$,

$$
\operatorname{cap}\left(\mathcal{E}_{k}, \mathcal{E}_{l}\right) \rightarrow 0 \quad \text { as } \quad l \rightarrow \infty .
$$

Choose $l$ big enough and rename $\mathcal{E}_{l}$ by $\mathcal{E}_{k+1}$, skipping all $\mathcal{E}_{i}$ with $k<i<l$.

Let $u_{k}$ be a capacity potential of the capacitor $\left(\mathcal{E}_{k}, \mathcal{E}_{k+1}\right), k \geq 1$, and let us set

$$
v(x)= \begin{cases}k+1-u_{k}(x), & \text { if } x \in \mathcal{E}_{k+1} \backslash \mathcal{E}_{k} \\ 1, & \text { if } x \in \mathcal{E}_{1}\end{cases}
$$

The function $v$ is continuous on $\partial \mathcal{E}_{k}$ and harmonic otherwise (see Figure 17). If $r \in[k, k+1)$, then, by (4.16) and (7.17),

$$
\operatorname{flux}_{\partial B_{r}} v=-\operatorname{flux}_{\partial \mathcal{E}_{k}} u_{k}=\operatorname{cap}\left(\mathcal{E}_{k}, \mathcal{E}_{k+1}\right) \leq 1,
$$

whence (7.16) follows.

Strictly speaking, the function $v$ is not smooth. However, it is possible to smooth $v$ out near $\partial \mathcal{E}_{k}$ without violating (7.16).

Another proof can be obtained by Theorem 5.5, which claims that, on a parabolic manifold, there exists an exhaustion function $v$ which is harmonic outside a compact. Therefore, for $r$ large enough, $\operatorname{flux}_{\partial B_{r}} v$ does not depend on $r$, whence we obtain (7.16).

If $v$ is an exhaustion function which is subharmonic outside a compact, then $\operatorname{flux}_{\partial B_{r}} v$ is decreasing in $r$, which again implies (7.16). Thus, we have the following

Corollary 7.7. If there exists an exhaustion function $v(x)$ on $M$, which is subharmonic outside a compact, then $M$ is parabolic.

Note that the word "subharmonic" can be replaced here by "superharmonic", by Corollary 5.4, but the proof of the latter was entirely different!

Let us discuss to what extent the hypotheses (7.12) and (7.15) of Theorems 7.3 and 7.5 are sharp. If the manifold $M$ has a non-negative Ricci curvature, then the condition (7.12) is also necessary for, and thus is equivalent to, parabolicity. This was proved by Varopoulos [181] and also follows from the Green function estimate 


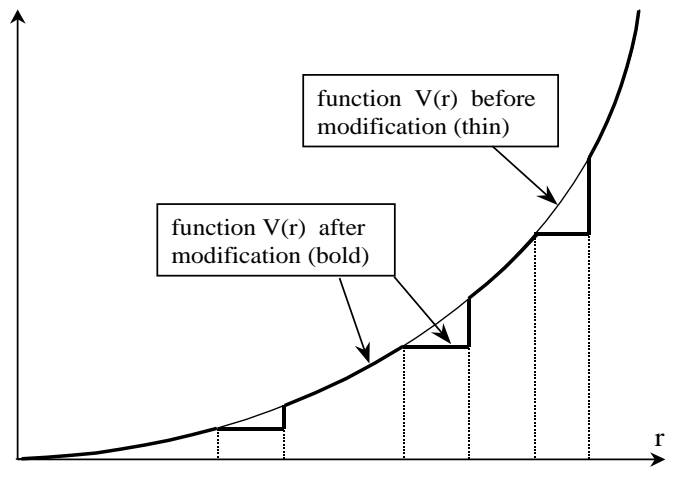

FigURE 18. Function $V(r)$ satisfying (7.19) and (7.21)

of Li and Yau [126],

$$
C^{-1} \int_{r}^{\infty} \frac{\rho d \rho}{V(x, \rho)} \leq G(x, y) \leq C \int_{r}^{\infty} \frac{\rho d \rho}{V(x, \rho)},
$$

where $r=\operatorname{dist}(x, y)$ and $C>0$. Indeed, the condition (7.12) is equivalent to $G \equiv \infty$, which in turn is equivalent to the parabolicity of $M$. Estimates (7.18) were also obtained by Varopoulos [182] for manifolds with non-negative sectional curvature.

In Section 11, we will see other situations when (7.12) is equivalent to parabolicity. However, in general, neither (7.12) nor (7.15) is necessary for parabolicity as is shown in the following examples.

Example 7.2. Let $M$ be a geodesically complete and non-compact model manifold $M_{\sigma}$. By Proposition 3.1, the parabolicity of $M_{\sigma}$ is equivalent

$$
\int^{\infty} \frac{d r}{V^{\prime}(r)}=\infty
$$

where $V(r)=V(o, r)$. Let us compare (7.19) with the hypothesis

$$
\int^{\infty} \frac{r d r}{V(r)}=\infty
$$

If (7.20) holds, then $M_{\sigma}$ is parabolic by Theorem 7.3, whence (7.19) follows. However, (7.19) does not necessarily imply (7.20). The manifold $M_{\sigma}$ with such volume function is parabolic, but (7.20) and (7.12) fail to hold.

Let us construct a volume function $V(r)$ satisfying (7.19) and

$$
\int^{\infty} \frac{r d r}{V(r)}<\infty
$$

Indeed, start with any $V(r)$ satisfying (7.21) and diminish it on a rare set of infinite measure so that $V^{\prime}$ becomes nearly 0 on this set (see Figure 18). The integral (7.21) does not change much, whereas the integral in (7.19) may become divergent.

This example shows also that a parabolic manifold may have arbitrarily fast volume growth. Another example for that can also be found in [184, p.826]. 


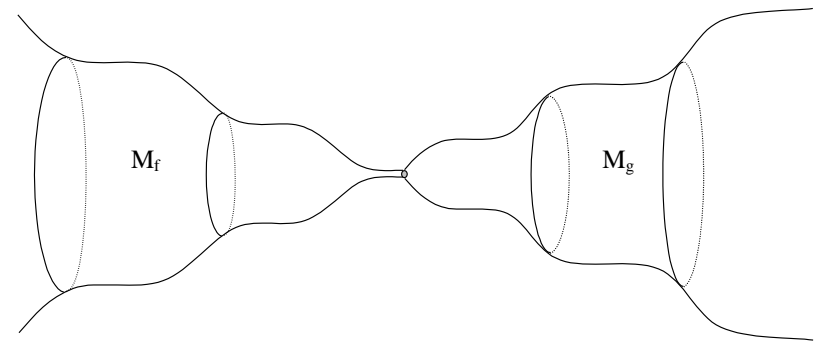

Figure 19. Connected sum of $M_{f}$ and $M_{g}$

If $V(r)$ is convex, then (7.21) does imply, and thus is equivalent to,

$$
\int^{\infty} \frac{d r}{V^{\prime}(r)}<\infty
$$

because $V^{\prime}(r) \geq \frac{V(r)-V(0)}{r}$. In particular, for a model manifold with a convex volume function $V(r)$, the condition (7.21) is equivalent to non-parabolicity.

Example 7.3. In general, the condition (7.15) is not necessary for parabolicity either. To see that, let us consider a couple of two-dimensional model manifolds $M_{f}$ and $M_{g}$ which are parabolic, that is

$$
\int^{\infty} \frac{d r}{f(r)}=\infty \text { and } \int^{\infty} \frac{d r}{g(r)}=\infty
$$

Let $M$ be a connected sum of $M_{f}$ and $M_{g}$ (see Figure 19). By Corollary 5.2, an exterior of a big enough compact in $M_{f}$ (and in $M_{g}$ ) is not massive. Therefore, an exterior of a big enough compact in $M$, being a disjoint union of such sets in $M_{f}$ and $M_{g}$, is not massive either, and $M$ is parabolic.

On the other hand, the boundary area function $S(x, r)$ on $M$ is equivalent to $f(r)+g(r)$ as $r \rightarrow \infty$. It is possible to find $f$ and $g$ satisfying, along with (7.22), also the condition

$$
\int^{\infty} \frac{d r}{f(r)+g(r)}<\infty
$$

Indeed, one can construct $f$ and $g$ so that $f(r)+g(r)=r^{2}$, for large $r$, but functions $f(r)$ and $g(r)$ may be chosen to stay nearly constant on the intervening intervals $(2 k, 2 k+1)$ and $(2 k+1,2 k+2)$, respectively, which makes the integrals in (7.22) divergent.

Therefore, we have

$$
\int^{\infty} \frac{d r}{S(x, r)}<\infty
$$

but $M$ is parabolic.

\section{Transience And Isoperimetric INEQUalities}

In this section, we obtain lower bounds of capacity and apply them to produce criteria of non-parabolicity. 


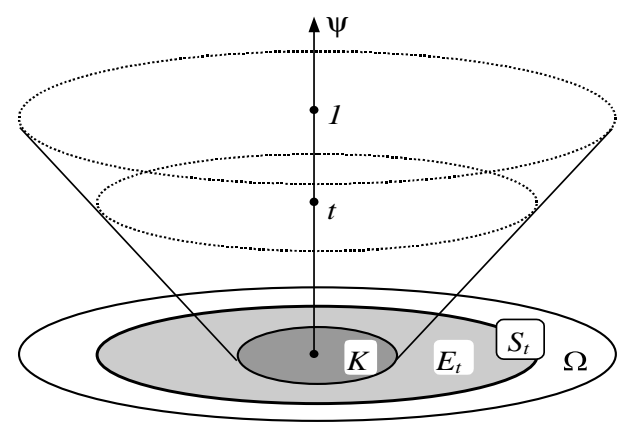

Figure 20. Function $\psi$ and sets $E_{t}, S_{t}$

Theorem 8.1. Let $\Omega \subset M$ be a precompact open set, and $K \subset \Omega$ be a compact set. Assume that for any region $E$ with a smooth boundary, such that $K \subset E \subset \Omega$, the inequality

$$
\mu^{\prime}(\partial E) \geq f(\mu(E))
$$

holds with a positive increasing function $f(v)$. Then the following estimate is true:

$$
\operatorname{cap}(K, \Omega)^{-1} \leq \int_{|K|}^{|\Omega|} \frac{d v}{f^{2}(v)}
$$

The estimate (8.2) was proved by Maz'ya [134, section 2.2.3] for an even more general notion of capacity in $\mathbb{R}^{d}$. For Riemannian manifolds, it was proved by the author [70], [71]. The inequality (8.2) is sharp in the sense that it becomes an equality if $K$ and $\Omega$ are concentric balls in $\mathbb{R}^{d}$.

Proof. Let $\phi \in \mathcal{D}(K, \Omega)$ where the class $\mathcal{D}(K, \Omega)$ is defined by (4.17), and let us set $\psi=1-\phi$. Since the Dirichlet integrals of $\phi$ and $\psi$ are equal, it suffices to prove that

$$
\int_{\Omega}|\nabla \psi|^{2} d \mu \geq\left\{\int_{|K|}^{|\Omega|} \frac{d v}{f^{2}(v)}\right\}^{-1} .
$$

For any $t \in(0,1)$, we denote (see Figure 20)

$$
S_{t}:=\{x \in \Omega: \psi(x)=t\} \quad \text { and } \quad E_{t}:=\{x \in \Omega: \psi(x)<t\} .
$$

Since $\psi \in C^{\infty}$, the Sard theorem implies that the set $S_{t}$ is a smooth hypersurface for almost all $t \in(0,1)$.

The coarea formula says that, for any continuous function $u$,

$$
\int_{\Omega \backslash K} u d \mu=\int_{0}^{1}\left\{\int_{S_{t}} u|\nabla \psi|^{-1} d \mu^{\prime}\right\} d t .
$$


We have by (8.4) with $u=|\nabla \psi|^{2}$ and by the Cauchy-Schwarz inequality

$$
\begin{aligned}
\int_{\Omega}|\nabla \psi|^{2} d \mu & =\int_{0}^{1}\left\{\int_{S_{t}}|\nabla \psi| d \mu^{\prime}\right\} d t \\
& \geq \int_{0}^{1} \frac{\left|S_{t}\right|^{2}}{\int_{S_{t}}|\nabla \psi|^{-1} d \mu^{\prime}} d t .
\end{aligned}
$$

Again, by (8.4) with $u=1$ (and with $E_{t}$ instead of $\Omega$ )

$$
v(t):=\left|E_{t}\right|=|K|+\int_{E_{t} \backslash K} d \mu=|K|+\int_{0}^{t}\left\{\int_{S_{\theta}}|\nabla \psi|^{-1} d \mu^{\prime}\right\} d \theta,
$$

whence for almost all $t \in(0,1)$

$$
v^{\prime}(t)=\int_{S_{t}}|\nabla \psi|^{-1} d \mu^{\prime} .
$$

By the isoperimetric inequality (8.1), we have for almost all $t \in(0,1)$

$$
\left|S_{t}\right| \geq f\left(\left|E_{t}\right|\right)=f(v(t))
$$

Therefore, we obtain from (8.5) and (8.6)

$$
\begin{aligned}
\int_{\Omega}|\nabla \psi|^{2} d \mu & \geq \int_{0}^{1} \frac{f^{2}(v(t))}{v^{\prime}(t)} d t \\
& \geq\left\{\int_{0}^{1} \frac{v^{\prime}(t) d t}{f^{2}(v(t))}\right\}^{-1} \geq\left\{\int_{v(0)}^{v(1)} \frac{d v}{f^{2}(v)}\right\}^{-1} \geq\left\{\int_{|K|}^{|\Omega|} \frac{d v}{f^{2}(v)}\right\}^{-1},
\end{aligned}
$$

whence (8.3).

Theorem 8.2. Assume that, for any precompact region $\Omega \subset M$ with a smooth boundary, the following inequality holds

$$
|\partial \Omega| \geq f(|\Omega|),
$$

where $f$ is a positive increasing function on $(0, \infty)$ such that

$$
\int^{\infty} \frac{d v}{f^{2}(v)}<\infty
$$

Then $M$ is non-parabolic.

This criterion was proved by Fernández [61] and by the author [70], [71]. In $\mathbb{R}^{d}$, inequality (8.7) holds with $f(v)=c_{d} v^{\frac{d-1}{d}}$. For such a function, (8.8) is satisfied if and only if $d>2$. 
Proof. The capacity estimate (8.2) and condition (8.8) imply that for any compact $K$ with positive measure,

$$
\operatorname{cap}(K) \geq\left\{\int_{|K|}^{|M|} \frac{d v}{f^{2}(v)}\right\}^{-1} \geq\left\{\int_{|K|}^{\infty} \frac{d v}{f^{2}(v)}\right\}^{-1}>0,
$$

whence by Theorem 5.1, $M$ is non-parabolic.

Another proof using the heat kernel argument will follow from Theorem 10.2 below. However, the capacity argument has its own value since it may be applied to other settings where the heat kernel is not available. For example, there is a generalization of the parabolicity test (7.12) for the non-linear elliptic equation $\operatorname{div}\left(|\nabla u|^{p-2} \nabla u\right)=0$ which is the Euler-Lagrange equation for the functional $\int|\nabla u|^{p}$; see [110], [95], [96], [39].

The estimates of capacities given by Theorems 7.1 and 8.1 can also be used to produce pointwise estimates of the Green function $G(x, y)$. Indeed, for any precompact open set $\Omega$ containing $y$ we have the inequalities

$$
\sup _{x \in \partial \Omega} G(x, y) \geq \operatorname{cap}(\Omega)^{-1} \geq \inf _{x \in \partial \Omega} G(x, y) .
$$

If one knows a Harnack inequality to relate $\sup _{x \in \partial \Omega} G(x, y)$ and $\inf _{x \in \partial \Omega} G(x, y)$, then one can get pointwise estimates of $G(x, y)$. See for details [127], [71]. See [23] and [20] for estimates of the volume of level sets of $G$ in connection with isoperimetric inequalities.

\section{Non-eXPLOSION AND VOLUme GROWTH}

We prove here the following test for non-explosion.

Theorem 9.1. Let $M$ be a geodesically complete manifold. If, for some point $x_{0} \in M$,

$$
\int^{\infty} \frac{r d r}{\log V\left(x_{0}, r\right)}=\infty
$$

then $M$ is stochastically complete.

Condition (9.1) holds, in particular, if

$$
V\left(x_{0}, r\right) \leq \exp \left(C r^{2}\right)
$$

for all $r$ large enough or even if $V\left(x_{0}, r_{k}\right) \leq \exp \left(C r_{k}^{2}\right)$, for a sequence $r_{k} \rightarrow \infty$ as $k \rightarrow \infty$.

Theorem 9.1 was proved by the author [72]. Other related results (all for geodesically complete manifolds) and references are as follows.

- M.Gaffney [66] proved that $\log V\left(x_{0}, r\right)=o(r), r \rightarrow \infty$, implies stochastic completeness.

- S.-T.Yau [193] proved that any manifold with Ricci curvature bounded below is stochastically complete.

- K.Ichihara [100] and P.Hsu [97] extended the above result of Yau to allow the Ricci curvature to grow in the negative direction in a certain way (see Corollary 15.4(a) below). 
- Karp and Li [109] proved that (9.2) is sufficient for stochastic completeness. This fact was proved also by different methods by E.B.Davies [44] and M.Takeda [177].

- Theorem 9.1 was extended by T.Sturm [173] to a general setting of Dirichlet spaces.

- See Section 15 for conditions for stochastic completeness in terms of the curvature.

Proof of Theorem 9.1. By Theorem 6.2, it suffices to verify that the only bounded solution to the Cauchy problem

$$
\left\{\begin{array}{l}
\frac{\partial}{\partial t} u-\frac{1}{2} \Delta u=0 \\
\left.u\right|_{t=0+}=0
\end{array}\right.
$$

in $M \times(0, T)$ (for some $T>0)$ is $u \equiv 0$. The function $u(x, t)$ is assumed to be in $C^{2}(M \times(0, T))$ and the initial data is understood in the sense of $L_{l o c}^{1}(M, \mu)$.

The assertion will follow from the following even more general fact.

Theorem 9.2. Let $M$ be a geodesically complete manifold, and let $u(x, t)$ be a bounded solution to the Cauchy problem (9.3) in $M \times(0, T)$, with the initial condition in the sense of $L_{l o c}^{2}(M, \mu)$. Assume that, for some $x_{0} \in M$ and for all $R$ large enough,

$$
\int_{0}^{T} \int_{B\left(x_{0}, R\right)} u^{2}(x, t) d \mu(x) d t \leq \exp (f(R)),
$$

where $f(r)$ is a positive monotone increasing function on $(0,+\infty)$ such that

$$
\int^{\infty} \frac{r d r}{f(r)}=\infty
$$

Then $u \equiv 0$ in $M \times(0, T)$.

Before the proof of Theorem 9.2, let us show why it implies Theorem 9.1. Let $u$ be a solution in $M \times(0, \infty)$ of the Cauchy problem (9.3), such that $\sup |u| \leq C$. In particular, $u$ satisfies the initial condition not only in the sense of $L_{l o c}^{1}(M, \mu)$ but also in the sense of $L_{l o c}^{2}(M, \mu)$. Assume that the hypothesis (9.1) holds. Then we have

$$
\int_{0}^{T} \int_{B\left(x_{0}, R\right)} u^{2}(x, t) d \mu(x) \leq C^{2} T V\left(x_{0}, R\right) .
$$

Denote

$$
f(r):=\log \left(C^{2} T V\left(x_{0}, r\right)\right)
$$

so that the hypothesis (9.4) is satisfied. Obviously, (9.5) is implied by (9.1). Hence, we can apply Theorem 9.2 which yields $u \equiv 0$.

Theorem 9.2 provides a uniqueness class (9.4) for the Cauchy problem. It can be regarded as a generalization of the classical uniqueness classes of Tichonov [178] and Täcklind [176] for the heat equation in $\mathbb{R}^{d}$. Indeed if, for example, the function $u(x, t)$ is a solution to the Cauchy problem (9.3) in $\mathbb{R}^{d}$ and belongs to the Tichonov class:

$$
|u(x, t)| \leq C \exp \left\{C|x|^{2}\right\}
$$




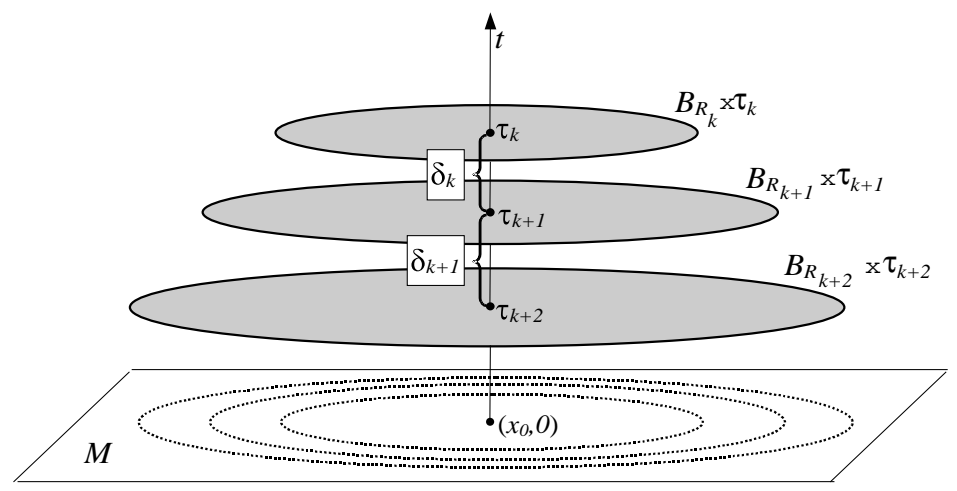

Figure 21. Sequences $\tau_{k}, \delta_{k}$ and $B_{R_{k}}$

then the conditions (9.4) and (9.5) are satisfied with $f(r)=(C+\varepsilon) r^{2}$, and we conclude, by Theorem 9.2 , that $u \equiv 0$.

Similar integral uniqueness classes for parabolic equations in unbounded domains in $\mathbb{R}^{d}$ were introduced by Oleinik and Radkevich [154] and by Gushchin [91]. See also [114], [143], [101] for the results on uniqueness for positive solutions .

Proof of Theorem 9.2. Given a large enough $R>0$ and $\tau \in(0, T)$, we will prove that for any $\delta \in(0, \tau]$ such that

$$
\delta \leq \frac{R^{2}}{c f(2 R)}
$$

the following inequality holds:

$$
\int_{B_{R}} u^{2}(x, \tau) d \mu(x) \leq \int_{B_{2 R}} u^{2}(x, \tau-\delta) d \mu(x)+\frac{c}{R^{2}},
$$

where $c>0$ is a (large) absolute constant. Suppose that we have proved (9.7) already; let us show how to derive $u \equiv 0$. Fix some (large) $R>0$ and $\tau \in(0, T)$. We define the sequence of radii $R_{k}=2^{k} R$ and the sequence $\left\{\delta_{k}\right\}, k=0,1,2, \ldots$ to satisfy (9.6) with $R=R_{k}$, that is,

$$
0<\delta_{k} \leq \frac{R_{k+1}^{2}}{4 c f\left(R_{k+1}\right)} .
$$

Let us define also the decreasing sequence $\left\{\tau_{k}\right\}$ inductively: $\tau_{0}=\tau$ and $\tau_{k+1}=$ $\tau_{k}-\delta_{k}$ (see Figure 21). The inequality (9.7) yields

$$
\int_{B_{R_{k}}} u^{2}\left(x, \tau_{k}\right) d \mu(x) \leq \int_{B_{R_{k+1}}} u^{2}\left(x, \tau_{k+1}\right) d \mu(x)+\frac{c}{R_{k}^{2}} .
$$

We would like the sequence $\left\{\tau_{k}\right\}$ to reach 0 for some finite $k$. Suppose that $\tau_{K+1}=0$; then

$$
\int_{B_{R_{K}}} u^{2}\left(x, \tau_{K+1}\right) d \mu(x)=0,
$$




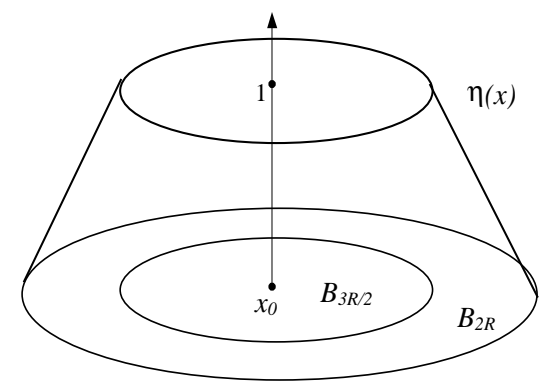

FiguRe 22. Function $\eta(x)$

and by iterating (9.9) up to $k=K$, we get

$$
\int_{B_{R}} u^{2}(x, \tau) d \mu(x) \leq \sum_{k=0}^{K} \frac{c}{R_{k}^{2}}<\frac{2 c}{R^{2}} .
$$

By letting $R \rightarrow \infty$, we conclude $u(\cdot, \tau) \equiv 0$. If we manage to prove this for any $\tau \in(0, T)$, then we will conclude $u \equiv 0$. Thus, we have to verify that, for any $\tau \in(0, T)$, the sequence $\left\{\tau_{k}\right\}$ can reach 0 in a finite number of steps. In other words, this means that, for some $K$,

$$
\tau=\delta_{0}+\delta_{1}+\delta_{2}+\ldots+\delta_{K}
$$

The only restriction on $\delta_{k}$ is the inequality (9.8), which imposes the upper bound on $\delta_{k}$. However, the hypothesis (9.5) implies that

$$
\sum_{k} \frac{R_{k}^{2}}{f\left(R_{k}\right)}=\infty
$$

Therefore, the sequence $\left\{\delta_{k}\right\}$ can be chosen to satisfy simultaneously (9.8) and

$$
\sum_{k} \delta_{k}=\infty
$$

By diminishing some of $\delta_{k}$, we can achieve (9.10) for any positive $\tau$.

Now we turn to the proof of $(9.7) .{ }^{8}$ Let $\rho(x)$ be a Lipschitz function on $M$ such that $|\nabla \rho| \leq 1$. For example, $\rho$ can be a distance function from a subset of $M$. Consider the function

$$
\xi(x, t):=\frac{\rho^{2}(x)}{2(t-s)}
$$

defined for all $x \in M$ and $t \neq s$ (where $s$ is a fixed number). It follows from $|\nabla \rho| \leq 1$ that $\xi$ satisfies the inequality

$$
\frac{\partial}{\partial t} \xi+\frac{1}{2}|\nabla \xi|^{2} \leq 0
$$

Let us denote for simplicity $B_{R}:=B\left(x_{0}, R\right)$. Let $\eta$ be a Lipschitz function such that $\eta(x)=0$ outside $B_{2 R}$ and $\eta=1$ in $B_{\frac{3}{2} R}$ (see Figure 22).

\footnotetext{
${ }^{8} \mathrm{~A}$ different proof can be found in [91].
} 
We multiply the equation (9.3) by $u \eta^{2} e^{\xi}$ and integrate over the cylinder $\mathcal{C}:=$ $B_{2 R} \times[\tau-\delta, \tau]$ for some $\delta \in(0, \tau)$ :

$$
2 \int_{\mathcal{C}} \frac{\partial u}{\partial t} u \eta^{2} e^{\xi} d \mu d t=\int_{\mathcal{C}} \Delta u \cdot u \eta^{2} e^{\xi} d \mu d t
$$

Next we integrate by parts in both sides of (9.12). The left hand side is equal to

$$
\int_{\tau-\delta}^{\tau} \int_{B_{2 R}} \frac{\partial\left(u^{2}\right)}{\partial \tau} \eta^{2} e^{\xi} d \mu d t=\left.\int_{B_{2 R}} u^{2} \eta^{2} e^{\xi} d \mu\right|_{\tau-\delta} ^{\tau}-\int_{\mathcal{C}} u^{2} \eta^{2} \frac{\partial \xi}{\partial t} e^{\xi} d \mu d t .
$$

The spatial integral on the right hand side of (9.12) is estimated as (we suppress $d \mu$ for simplicity)

$$
\begin{aligned}
\int_{B_{2 R}} \Delta u \cdot u \eta^{2} e^{\xi}= & -\int_{B_{2 R}}|\nabla u|^{2} \eta^{2} e^{\xi}-2 \int_{B_{2 R}} \nabla u \nabla \eta u \eta e^{\xi}-\int_{B_{2 R}} \nabla u \nabla \xi u \eta^{2} e^{\xi} \\
\leq & -\int_{B_{2 R}}|\nabla u|^{2} \eta^{2} e^{\xi}+2 \int_{B_{2 R}}\left(\frac{1}{4}|\nabla u|^{2} \eta^{2}+|\nabla \eta|^{2} u^{2}\right) e^{\xi} \\
& +\int_{B_{2 R}}\left(\frac{1}{2}|\nabla u|^{2}+\frac{1}{2}|\nabla \xi|^{2} u^{2}\right) \eta^{2} e^{\xi} \\
= & 2 \int_{B_{2 R}}|\nabla \eta|^{2} u^{2} e^{\xi}+\frac{1}{2} \int_{B_{2 R}}|\nabla \xi|^{2} u^{2} \eta^{2} e^{\xi} .
\end{aligned}
$$

Therefore, we obtain from (9.12):

$$
\left.\int_{B_{2 R}} u^{2} \eta^{2} e^{\xi} d \mu\right|_{\tau-\delta} ^{\tau} \leq \int_{\mathcal{C}}\left\{\eta^{2} \frac{\partial \xi}{\partial t}+2|\nabla \eta|^{2}+\frac{1}{2}|\nabla \xi|^{2} \eta^{2}\right\} u^{2} e^{\xi} d \mu d t .
$$

Due to inequality (9.11), the first and the third terms of the right hand side of (9.13) cancel, and we obtain

$$
\left.\int_{B_{2 R}} u^{2} \eta^{2} e^{\xi} d \mu\right|_{\tau-\delta} ^{\tau} \leq 2 \int_{\mathcal{C}}|\nabla \eta|^{2} u^{2} e^{\xi} d \mu d t
$$

The function $\eta$ can be chosen so that $\eta \leq 1$ and $|\nabla \eta| \leq \frac{C}{R}$ for some constant $C$ independent on $R$ (geodesic completeness is required for existence of such $\eta$ ). Taking into account that $\eta=1$ on $B_{\frac{3}{2} R}$, we can rewrite (9.14) as

$$
\begin{aligned}
\int_{B_{R}} u^{2}(x, \tau) e^{\xi(x, \tau)} d \mu(x) \leq & \int_{B_{2 R}} u^{2}(x, \tau-\delta) e^{\xi(x, \tau-\delta)} d \mu(x) \\
& +\frac{2 C^{2}}{R^{2}} \int_{\tau-\delta}^{\tau} \int_{B_{2 R} \backslash B_{3 R / 2}} u^{2} e^{\xi} d \mu d t .
\end{aligned}
$$


Now we specify $\rho(x)$ and $\xi(x, t)$. Let us choose $\rho(x)$ to be the distance function from the ball $B_{R}$; i.e.

$$
\rho(x):=\left\{\begin{array}{lr}
0, & \text { if } x \in B_{R} \\
\operatorname{dist}\left(x, x_{0}\right)-R, & \text { otherwise. }
\end{array}\right.
$$

Also, let $s=\tau+\delta$ so that for $t \in(\tau-\delta, \tau)$

$$
\xi(x, t)=-\frac{\rho^{2}(x)}{2(\tau+\delta-t)} \leq 0 .
$$

In particular, we can omit the factor $e^{\xi}$ on the left hand side of (9.15) because $\xi=0$ when $x \in B_{R}$, and omit the factor $e^{\xi}$ in the first integral on the right hand side of (9.15) for $\xi \leq 0$. Since, for $x \in B_{2 R} \backslash B_{3 R / 2}$,

$$
\rho(x) \geq \frac{1}{2} R
$$

and, for $t \in(\tau-\delta, \tau)$,

$$
s-t=\tau+\delta-t \leq 2 \delta,
$$

we obtain for those $x$ and $t$

$$
\xi(x, t) \leq-\frac{1}{16} \frac{R^{2}}{\delta}
$$

We derive from (9.15)

$$
\int_{B_{R}} u^{2}(x, \tau) d \mu(x) \leq \int_{B_{2 R}} u^{2}(x, \tau-\delta) d \mu(x)+\frac{2 C^{2}}{R^{2}} \int_{\mathcal{C}} u^{2} \exp \left\{-\frac{1}{16} \frac{R^{2}}{\delta}\right\} d \mu d t .
$$

Finally, we apply the hypothesis (9.4) which implies

$$
\int_{\mathcal{C}} u^{2}(x, t) d \mu(x) d t \leq \exp (f(2 R))
$$

and, hence,

$$
\int_{B_{R}} u^{2}(x, \tau) d \mu(x) \leq \int_{B_{2 R}} u^{2}(x, \tau-\delta) d \mu(x)+\frac{2 C^{2}}{R^{2}} \exp \left\{-\frac{1}{16} \frac{R^{2}}{\delta}+f(2 R)\right\} .
$$

Now we choose $\delta$ so small that

$$
\frac{1}{16} \frac{R^{2}}{\delta} \geq f(2 R)
$$

or

$$
\delta \leq \frac{R^{2}}{16 f(2 R)}
$$

For such $\delta$, we have

$$
\int_{B_{R}} u^{2}(x, \tau) d \mu(x) \leq \int_{B_{2 R}} u^{2}(x, \tau-\delta) d \mu(x)+\frac{2 C^{2}}{R^{2}}
$$

which coincides with (9.7). 


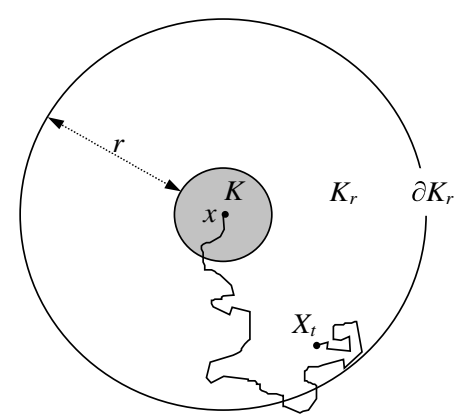

Figure 23. The event that the Brownian motion hits $\partial K_{r}$ before the time $t$

In the rest of this section, we briefly discuss another approach for proving stochastic completeness. Let us assume that $M$ is geodesically complete, and that for some $x_{0}$ and for all large enough $r$ :

$$
V\left(x_{0}, r\right) \leq \exp \left(C r^{2}\right) .
$$

Takeda's approach [177] is based on the following remarkable inequality which was established in [177] and improved by Lyons [130]. For any compact set $K \subset M$, we take $K_{r}$ to be the open $r$-neighborhood of $K$. We denote by $\psi_{r}(x, t)$ the $\mathbb{P}_{x^{-}}$ probability that the process $X_{t}$ hits $M \backslash K_{r}$ by time $t$ (see Figure 23); i.e.

$$
\psi_{r}(x, t):=\mathbb{P}_{x}\left\{\exists s \in[0, t]: X_{s} \notin K_{r}\right\} .
$$

Theorem 9.3. (Takeda [177], Lyons [130]) Let $M$ be geodesically complete. Then, for any compact $K$ and all $t>0, r>0$,

$$
\int_{K} \psi_{r}(x, t) d \mu(x) \leq 16\left|K_{r}\right| \int_{r}^{\infty} \frac{1}{(2 \pi t)^{1 / 2}} \exp \left\{-\frac{\xi^{2}}{2 t}\right\} d \xi .
$$

Remark. A similar estimate of the $L^{2}$-norm of $\psi_{r}$ can be found in [81].

Inequality (9.19) implies easily

$$
\int_{K} \psi_{r}(x, t) d \mu(x) \leq 16\left|K_{r}\right| \exp \left\{-\frac{r^{2}}{2 t}\right\}
$$

and

$$
\inf _{x \in K} \psi_{r}(x, t) \leq 16 \frac{\left|K_{r}\right|}{|K|} \exp \left\{-\frac{r^{2}}{2 t}\right\} .
$$

Given (9.18), we see that, for $r$ large enough, $\left|K_{r}\right| \leq \exp \left\{C^{\prime} r^{2}\right\}$, which together with (9.20) implies that, for $t<t_{0}:=\left(2 C^{\prime}\right)^{-1}$,

$$
\inf _{x \in K} \psi_{r}(x, t) \underset{r \rightarrow \infty}{\longrightarrow} 0 .
$$

Intuitively, this is enough to conclude stochastic completeness. Indeed, (9.21) means that, for some point $x \in K$, the $\mathbb{P}_{x}$-probability that $X_{t}$ reaches the boundary $\partial K_{r}$ by time $t$ is very small for large $r$. This leads us to believe that the $\mathbb{P}_{x}$-probability that $X_{t}$ escapes to $\infty$ in finite time should be 0 . 
To make this argument rigorous, consider the heat kernel $p_{K_{r}}(t, x, y)$ of $K_{r}$ with the Dirichlet condition on the boundary $\partial K_{r}$. The integral

$$
\int_{K_{r}} p_{K_{r}}(t, x, y) d \mu(y)
$$

is equal to the $\mathbb{P}_{x}$-probability that $X_{t}$ stays in $K_{r}$ up to time $t$, without touching the boundary $\partial K_{r}$. Therefore,

$$
\psi_{r}(x, t)+\int_{K_{r}} p_{K_{r}}(t, x, y) d \mu(y)=1,
$$

which yields together with $(9.21)$ and $p(t, x, y) \geq p_{K_{r}}(t, x, y)$

$$
\sup _{x \in K} \int_{M} p(t, x, y) d \mu(y)=1, \quad \forall t \in\left(0, t_{0}\right) .
$$

By Theorem 6.2(1a), $M$ is stochastically complete.

\section{TRansience and $\lambda_{1}$}

The main result of this section is Theorem 10.2, which provides a non-parabolicity test in terms of certain property of the eigenvalues of the Laplace operator.

10.1. The first eigenvalue. For any precompact open set $\Omega \subset M$, let us denote by $\lambda_{1}(\Omega)$ the first Dirichlet eigenvalue of the Laplace operator in $\Omega$; i.e. $\lambda_{1}(\Omega)$ is the smallest number $\lambda$ for which the problem

$$
\left\{\begin{array}{l}
\Delta u+\lambda u=0 \text { in } \Omega \\
\left.u\right|_{\partial \Omega}=0
\end{array}\right.
$$

has a non-zero (weak) solution. Another (equivalent) definition is the following: $\lambda_{1}(\Omega)$ is the bottom of the spectrum of the operator $-\Delta$ in $L^{2}(\Omega, \mu)$ with the domain $C_{0}^{\infty}(\Omega)$. The latter definition is independent of compactness of $\Omega$, so it applies for any region $\Omega$. In particular, $\Omega$ may be the whole space $M$. Yet another definition of $\lambda_{1}(\Omega)$ is given by the variational principle:

$$
\lambda_{1}(\Omega)=\inf _{\substack{\phi \in C_{0}^{\infty}(\Omega) \\ \phi \neq 0}} \frac{\int_{\Omega}|\nabla \phi|^{2} d \mu}{\int_{\Omega} \phi^{2} d \mu} .
$$

It is easy to show that $\lambda_{1}(\Omega)$ is non-negative, that it decreases when $\Omega$ increases, and that $\lambda_{1}\left(\mathcal{E}_{k}\right) \underset{k \rightarrow \infty}{\longrightarrow} \lambda_{1}(M)$ for any exhaustion sequence $\left\{\mathcal{E}_{k}\right\}$.

Example 10.1. We have in $\mathbb{R}^{d}$ for any ball $\lambda_{1}(B(x, R))=\frac{c_{d}}{R^{2}}$ and $\lambda_{1}\left(\mathbb{R}^{d}\right)=0$. For the hyperbolic space $\lambda_{1}\left(\mathbb{H}^{d}\right)=\frac{(d-1)^{2}}{4}$.

A theorem of McKean [137] says that if $M$ is a Cartan-Hadamard manifold ${ }^{9}$ of dimension $d$ and if its sectional curvature is bounded above by $-k^{2}$, then

$$
\lambda_{1}(M) \geq \frac{1}{4}(d-1)^{2} k^{2}
$$

(see also [192]).

${ }^{9} \mathrm{~A}$ manifold is called a Cartan-Hadamard manifold if it is geodesically complete, simply connected and has non-positive sectional curvature. Both $\mathbb{R}^{d}$ and $\mathbb{H}^{d}$ are Cartan-Hadamard manifolds. 
A theorem of Brooks [18] gives the following relation between $\lambda_{1}(M)$ and volume growth of a geodesically complete manifold. If we denote, for a fixed $x$,

$$
\nu:=\limsup _{r \rightarrow \infty} \frac{\log V(x, r)}{r},
$$

then $^{10}$

$$
\lambda_{1}(M) \leq \frac{\nu^{2}}{4}
$$

In particular, $\lambda_{1}(M)=0$ for manifolds with subexponential volume growth. For the manifolds $\mathbb{R}^{d}$ and $\mathbb{H}^{d}$, both inequalities (10.2), (10.3) become equalities.

There is a well-known universal connection between $\lambda_{1}(M)$ and the heat kernel long time behaviour: for all $x, y \in M$,

$$
\lim _{t \rightarrow \infty} \frac{\log p(t, x, y)}{t}=-\frac{1}{2} \lambda_{1}(M)
$$

see [118] and [25]. It implies immediately

Proposition 10.1. If $\lambda_{1}(M)>0$ then $M$ is non-parabolic.

Indeed, by (10.4), the heat kernel $p(t, x, y)$ decays in $t$ exponentially, whence the integral (5.1) is convergent and $M$ is non-parabolic, by Theorem 5.1(5).

There are interesting applications of the discrete analogue of this fact in the paper of Dodziuk [49].

Remark. In the view of Proposition 10.1, one may wonder if $\lambda_{1}(M)>0$ implies a stronger property than non-parabolicity, for example, existence of a non-trivial bounded harmonic function on $M$ (cf. Theorem 5.1(3)). The answer is negative. There is a beautiful example of Benjamini and Cao [11] of a geodesically complete manifold which is simply connected, has $\lambda_{1}(M)>0$ and bounded geometry but admits no non-constant bounded harmonic function.

See [119, Section 13] for a survey on the existence of a non-trivial bounded harmonic function on Cartan-Hadamard manifolds.

10.2. Faber-Krahn inequality and transience. In this section, we prove a farreaching extension of Proposition 10.1. Given a positive decreasing function $\Lambda(\cdot)$ on $(0, \infty)$, we say that a manifold $M$ satisfies a Faber-Krahn inequality with function $\Lambda$ if, for any precompact region $\Omega \subset M$,

$$
\lambda_{1}(\Omega) \geq \Lambda(|\Omega|) .
$$

This is motivated by the Faber-Krahn theorem, which says that, for any bounded region $\Omega \subset \mathbb{R}^{d}$,

$$
\lambda_{1}(\Omega) \geq c_{d}|\Omega|^{-\frac{2}{d}}
$$

(see, for example, [21, Chapter IV]), with the constant $c_{d}$ such that equality in (10.6) is attained for balls. In other words, $\mathbb{R}^{d}$ satisfies a Faber-Krahn inequality with function $\Lambda(v)=c_{d} v^{-\frac{2}{d}}$.

Other examples of Faber-Krahn inequalities will be given below. In general, a Faber-Krahn inequality need not be as sharp as (10.6) - in particular, we will not use the exact value of $c_{d}$.

\footnotetext{
${ }^{10}$ In fact, Brooks' theorem states that even $\lambda_{\text {ess }}(M) \leq \frac{\nu^{2}}{4}$ where $\lambda_{\text {ess }}(M)$ is the bottom of the essential spectrum of $-\Delta$.
} 
Theorem 10.2. ([80, Theorem 2.3]) Let $\Lambda(v)$ be a positive monotone decreasing function on $(0, \infty)$ such that

$$
\int^{\infty} \frac{d v}{v^{2} \Lambda(v)}<\infty
$$

Assume that the Faber-Krahn inequality (10.5) holds for all precompact open sets $\Omega \subset M$ with large enough volume, say $|\Omega| \geq v_{0}$. Then $M$ is non-parabolic.

Remark. Formally, the hypotheses of Theorem (10.2) allow the manifold $M$ to have finite volume. In this case $\Lambda(v)$ is extended for $v>|M|$ by the constant $\Lambda(|M|)$. The condition (10.7) amounts in this case to $\lambda_{1}(M)>0$. However, the most interesting case of Theorem 10.2 is when $|M|=\infty$ so that (10.7) is a lower bound on the rate of the decay of $\Lambda$ at $\infty$. For example, if $\Lambda(v) \sim v^{-\beta}$ for large $v$, then (10.7) is satisfied if and only if $\beta<1$.

Theorem 10.2 contains Proposition 10.1. Indeed, if $\lambda_{1}(M)>0$ then we take $\Lambda(v) \equiv \lambda_{1}(M)$, which satisfies (10.7).

Theorem 10.2 contains also Theorem 8.2, at least if $\frac{f(v)}{v}$ is decreasing. Indeed, under the hypothesis of Theorem 8.2, we have, by Cheeger's inequality [26],

$$
\lambda_{1}(\Omega) \geq \frac{1}{4}\left(\inf _{D \subset \subset \Omega} \frac{|\partial D|}{|D|}\right)^{2} \geq \frac{1}{4}\left(\inf _{D \subset \subset \Omega} \frac{f(|D|)}{|D|}\right)^{2} \geq \frac{1}{4}\left(\frac{f(|\Omega|)}{|\Omega|}\right)^{2} .
$$

Therefore, $M$ admits a Faber-Krahn inequality with the function

$$
\Lambda(v):=\frac{1}{4}\left(\frac{f(v)}{v}\right)^{2} .
$$

Obviously, hypothesis (8.8) coincides with (10.7), whence $M$ is non-parabolic by Theorem 10.2.

Proof of Theorem 10.2. Let us first observe that, by modifying $\Lambda(v)$ for $v<v_{0}$, we can show that the Faber-Krahn inequality (10.5) holds for all $\Omega$, not only for those with large volume. Indeed, let us redefine $\Lambda(v):=\Lambda\left(v_{0}\right)$ for all $v<v_{0}$. Clearly, the hypothesis (10.7) does not change when we modify $\Lambda$ on $\left(0, v_{0}\right)$. If $|\Omega|<v_{0}$ then find a set $\Omega^{\prime} \supset \Omega$ of the volume $v_{0}$ and notice that, by the monotonicity of $\lambda_{1}(\Omega)$,

$$
\lambda_{1}(\Omega) \geq \lambda_{1}\left(\Omega^{\prime}\right) \geq \Lambda\left(\Omega^{\prime}\right)=\Lambda\left(v_{0}\right)=\Lambda(|\Omega|) .
$$

Henceforth, we can assume that the Faber-Krahn inequality (10.5) holds for all $\Omega$.

The following line of reasoning was designed to obtain pointwise upper bounds of the heat kernel via the Faber-Krahn function $\Lambda$ (see [91] and [80]). Since the non-parabolicity is equivalent to

$$
\int^{\infty} p(t, x, x) d t<\infty
$$

one gets a sufficient condition for non-parabolicity by integrating the upper bound of $p(t, x, x)$.

We start with the following consequence of the Faber-Krahn inequality (10.5).

Lemma 10.3. ([91], [80]) For any precompact region $\Omega \subset M$, for any non-negative function $u \in C^{2}(\Omega) \cap C(\bar{\Omega})$ vanishing on $\partial \Omega$, and for any $\delta \in(0,1)$, we have

$$
\int_{\Omega}|\nabla u|^{2} d \mu \geq(1-\delta) J \Lambda\left(\frac{2 I^{2}}{\delta J}\right)
$$


where

$$
I:=\int_{\Omega} u d \mu \quad \text { and } \quad J:=\int_{\Omega} u^{2} d \mu .
$$

Proof. For any positive $s$, we obviously have

$$
u^{2} \leq(u-s)_{+}^{2}+2 s u
$$

By integrating this inequality over $\Omega$ and using the definitions of $I$ and $J$, we obtain

$$
J \leq \int_{\{u>s\}}(u-s)^{2} d \mu+2 s I .
$$

Applying the variational property (10.1) of the first eigenvalue in the region $\Omega_{s}:=$ $\{u>s\}$ (observe that $u-s$ vanishes on the boundary $\partial \Omega_{s}$ ) and the Faber-Krahn inequality (10.5), we get

$$
\int_{\Omega_{s}}(u-s)^{2} d \mu \leq \frac{\int_{\Omega_{s}}|\nabla u|^{2} d \mu}{\Lambda\left(\left|\Omega_{s}\right|\right)} .
$$

Clearly,

$$
\left|\Omega_{s}\right| \leq \frac{1}{s} \int_{\Omega} u d \mu=\frac{I}{s}
$$

whence we obtain, by substituting into (10.11),

$$
J \leq \frac{\int_{\Omega_{s}}|\nabla u|^{2} d \mu}{\Lambda\left(s^{-1} I\right)}+2 s I
$$

and

$$
\int_{\Omega_{s}}|\nabla u|^{2} d \mu \geq(J-2 s I) \Lambda\left(s^{-1} I\right) .
$$

Choosing $s=\frac{\delta J}{2 I}$, we conclude (10.10).

Next, let us fix a point $y \in \Omega$, and introduce the notation

$$
u(x, t):=p_{\Omega}(t, x, y) \quad \text { and } \quad J(t):=\int_{\Omega} u^{2}(x, t) d \mu=p_{\Omega}(2 t, y, y) .
$$

Applying inequality (10.10) to the function $u(\cdot, t)$ and observing that

$$
I=\int_{\Omega} u(x, t)=\int_{\Omega} p_{\Omega}(t, x, y) d \mu \leq 1
$$

we obtain

$$
\int_{\Omega}|\nabla u|^{2} d \mu \geq(1-\delta) J(t) \Lambda\left(\frac{2}{\delta J(t)}\right) .
$$

The function $u(x, t)$ satisfies in $\Omega \times(0, \infty)$ the heat equation $\frac{\partial u}{\partial t}=\frac{1}{2} \Delta u$. By multiplying it by $u$ and by integrating over $\Omega$, we obtain

$$
\frac{\partial}{\partial t} \int_{\Omega} u^{2} d \mu=\int_{\Omega} u \Delta u=-\int_{\Omega}|\nabla u|^{2}
$$

which yields, together with (10.12), the differential inequality in the spirit of Nash's argument [147]:

$$
-\frac{\partial J}{\partial t} \geq(1-\delta) J(t) \Lambda\left(\frac{2}{\delta J(t)}\right) .
$$


To verify that $M$ is non-parabolic we have to find an upper bound for

$$
\int_{a}^{\infty} p_{\Omega}(2 t, y, y) d t=\int_{a}^{\infty} J(t) d t
$$

which would be independent of $\Omega$. By using (10.13) with $\delta=\frac{1}{2}$, we have, for all $b>a>0$

$$
\int_{a}^{b} J(t) d t=\int_{J(b)}^{J(a)}\left(-\frac{d t}{d J}\right) J d J \leq 2 \int_{J(b)}^{J(a)} \frac{d J}{\Lambda\left(\frac{4}{J}\right)}=8 \int_{\frac{4}{J(a)}}^{\frac{4}{J(b)}} \frac{d v}{v^{2} \Lambda(v)}
$$

where we have changed in the last integral $v=\frac{4}{J}$. Therefore, we have, for any $a>0$,

$$
\int_{a}^{\infty} p_{\Omega}(2 t, y, y) d t=\int_{a}^{\infty} J(t) d t \leq 8 \int_{\frac{4}{J(a)}}^{\infty} \frac{d v}{v^{2} \Lambda(v)}
$$

Now let $\Omega \uparrow M$. We have $p_{\Omega} \rightarrow p$ and $J(a)=p_{\Omega}(2 a, y, y) \leq p(2 a, y, y)$. Hence,

$$
\int_{a}^{\infty} p(2 t, y, y) d t \leq 8 \int_{\frac{4}{p(2 a, y, y)}}^{\infty} \frac{d v}{v^{2} \Lambda(v)}<\infty
$$

whence the non-parabolicity of $M$ follows.

10.3. Heat kernel's upper bound. One can resolve (10.13) to produce pointwise upper bounds of the heat kernel.

Proposition 10.4. Let the Faber-Krahn inequality (10.5) hold on M. Fix $x \in M$, $t_{0} \geq 0, \delta \in(0,1)$ and assume that a non-negative function $\Phi(t)$ on $\left[t_{0}, \infty\right)$ satisfies the following conditions:

$$
\begin{aligned}
\Phi\left(t_{0}\right) & \leq \frac{2}{\delta p\left(2 t_{0}, x, x\right)}, \\
\int_{\Phi\left(t_{0}\right)}^{\Phi(t)} \frac{d v}{v \Lambda(v)} & =(1-\delta)\left(t-t_{0}\right) .
\end{aligned}
$$

Then, for all $t>t_{0}$,

$$
p(2 t, x, x) \leq \frac{2}{\delta \Phi(t)} .
$$

Remark. If we let $t_{0}=0$ and $\Phi\left(t_{0}\right)=0$, then (10.14) is automatically satisfied. In order to have (10.15) one has to assume

$$
\int_{0} \frac{d v}{v \Lambda(v)}<\infty
$$

If this is the case, then the function $\Phi(t)$ is defined by the following identity

$$
\int_{0}^{\Phi(t)} \frac{d v}{v \Lambda(v)}=(1-\delta) t
$$

and (10.16) holds for all $x \in M$ (see also [80, Theorem 2.1]). 
However, if (10.17) is not true, then $t_{0}$ should be positive, and in order to obtain an upper bound of the heat kernel $p(2 t, x, x)$ for $t>t_{0}$, one should know a priori an upper bound for $p\left(2 t_{0}, x, x\right)$ (given by (10.14)).

Proof. Take some $\Omega$ containing $x$, denote

$$
J(t):=\int_{\Omega} p_{\Omega}^{2}(x, y, t) d \mu(y)=p_{\Omega}(2 t, x, x)
$$

and continue the previous argument as follows. Dividing (10.13) by the right-hand side, integrating in $t$ and changing variable $v=\frac{2}{\delta J(t)}$, we obtain, for all $t>t_{0}>0$,

$$
\int_{\frac{2}{\delta J\left(t_{0}\right)}}^{\frac{2}{\delta J(t)}} \frac{d v}{v \Lambda(v)} \geq(1-\delta)\left(t-t_{0}\right) .
$$

Hypothesis (10.14) implies

$$
\Phi\left(t_{0}\right) \leq \frac{2}{\delta p\left(2 t_{0}, x, x\right)} \leq \frac{2}{\delta p_{\Omega}\left(2 t_{0}, x, x\right)}=\frac{2}{\delta J\left(t_{0}\right)} .
$$

Comparing (10.19) and (10.15) we obtain that, for all $t>t_{0}$,

$$
\Phi(t) \leq \frac{2}{\delta J(t)}=\frac{2}{\delta p_{\Omega}(2 t, x, x)},
$$

whence

$$
p_{\Omega}(2 t, x, x) \leq \frac{2}{\delta \Phi(t)} .
$$

By letting $\Omega \rightarrow M$, we obtain (10.16).

Example 10.2. For the Euclidean Faber-Krahn function

$$
\Lambda(v)=c v^{-2 / d}
$$

one gets from $(10.18) \Phi(t)=$ const $(c t)^{2 / d}$ and from $(10.16)$

$$
p(t, x, x) \leq \frac{\mathrm{const}}{t^{d / 2}},
$$

for all $x \in M$ and $t>0$.

If the Faber-Krahn inequality with the function (10.21) holds only for large $v$, then as was explained above, we extend $\Lambda$ to all smaller $v$ by a constant. We cannot use (10.18) anymore because (10.17) is no longer true, but we can still use (10.15) to obtain (10.22), for $t$ large enough.

Example 10.3. Let

$$
\Lambda(v)=\frac{\text { const }}{\log ^{\alpha} v},
$$

for $v$ large enough. Then (10.15) yields, for $t$ large enough and some $c>0$,

$$
\Phi(t) \sim \exp \left\{c t^{\frac{1}{1+\alpha}}\right\}
$$

and

$$
p(t, x, x) \leq \text { const } \exp \left\{-c t^{\frac{1}{1+\alpha}}\right\}
$$


Example 10.4. Let $\Lambda(v) \equiv \lambda$ where $\lambda$ is a positive constant. Inequality (10.10) can then be replaced in the argument above by the much simpler

$$
\int_{\Omega}|\nabla u|^{2} \geq \lambda J
$$

which leads to $-J^{\prime} \geq \lambda J$ and eventually to

$$
p(t, x, x) \leq e^{-\frac{1}{2} \lambda\left(t-t_{0}\right)} p\left(t_{0}, x, x\right) .
$$

In particular, for $t$ large enough,

$$
p(t, x, x) \leq \text { const } \exp (-\lambda t) .
$$

There is a vast literature on upper bounds of the heat kernel: see for example [19], [34], [42], [46], [79], [80], [82], [126], [186].

\section{Transience and volume Growth}

In this section, we describe two situations when the non-parabolicity of $M$ follows from (and thus is equivalent to) the condition

$$
\int^{\infty} \frac{r d r}{V(x, r)}<\infty
$$

\subsection{Relative Faber-Krahn inequality.}

Theorem 11.1. Let $M$ be geodesically complete and non-compact. Assume that for any ball $B(x, R)$ and any region $\Omega \subset \subset B(x, R)$,

$$
\lambda_{1}(\Omega) \geq \frac{a}{R^{2}}\left(\frac{V(x, R)}{|\Omega|}\right)^{\nu}
$$

where $a>0$ and $\nu>0$ are some constants. Then (11.1) is equivalent to the non-parabolicity of $M$.

Inequality (11.2) may look strange at first sight. However, it is useful because, for example, it holds on any complete manifold of non-negative Ricci curvature as well as on any manifold which is quasi-isometric to one of non-negative Ricci curvature (see [78, Theorems 2.1 and 1.4] and discussion in [79, p.254]). It is easy to see that (11.2) is valid in $\mathbb{R}^{d}$ with $\nu=2 / d$ (cf. (10.6)). For manifolds of nonnegative Ricci curvature, the fact that (11.1) is equivalent to non-parabolicity was proved by Varopoulos [181].

Given a complete manifold of non-negative Ricci curvature, there may exist no positive function $\Lambda$ with which the Faber-Krahn inequality (10.5) would be valid on $M$. The inequality (11.2) was designed to overcome this difficulty. It basically says that, in each ball $B(x, R)$, there is still a Faber-Krahn inequality (10.5) with the function

$$
\Lambda_{x, R}(v)=\frac{a V(x, R)^{\nu}}{R^{2}} v^{-\nu} .
$$

Proof of Theorem 11.1. The proof follows immediately from [80, Proposition 5.2], which says in particular that (11.2) implies the following upper bound of the heat kernel:

$$
p(t, x, x) \leq \frac{\text { const }}{V(x, \sqrt{t})} .
$$


Therefore, if (11.1) is true, then

$$
\int^{\infty} p(t, x, x) d t \leq \int^{\infty} \frac{\text { const }}{V(x, \sqrt{t})} d t=\text { const } \int^{\infty} \frac{r d r}{V(x, r)}<\infty,
$$

whence $M$ is non-parabolic.

If (11.1) is not true, then $M$ is parabolic by Theorem 7.3. Alternatively, it follows also from the lower bound

$$
p(t, x, x) \geq \frac{\text { const }}{V(x, \sqrt{t})},
$$

which is implied by (11.2) - see [36, Corollary 7.3].

Remark. The relative Faber-Krahn inequality (11.2) implies also the doubling property of the volume function $V(x, r)$, that is, $V(x, 2 r) \leq C V(x, r)$. Moreover, the doubling property and the heat kernel upper bound (11.4) are equivalent to (11.2) - see [80, Proposition 5.2], [37, Theorem 1.1].

Remark. The relative Faber-Krahn inequality (11.2) together with the hypothesis

$$
V(x, r) \geq \text { const } r^{n}, \quad \forall x \in M, r>0,
$$

implies a uniform Faber-Krahn inequality

$$
\lambda_{1}(\Omega) \geq \text { const }|\Omega|^{-2 / n} .
$$

Indeed, (11.2) implies the upper bound (11.4) of the heat kernel, which together with (11.6) yields

$$
p(t, x, y) \leq \frac{\text { const }}{t^{n / 2}}
$$

Finally, (11.8) implies (11.7) by [80, Theorem 2.2] or [20, Theorem 0.7].

Remark. Let us fix a point $x \in M$ and a radius $R$ and interpret the ball $B(x, R)$ as a manifold $N$ which admits a uniform Faber-Krahn inequality (10.5) with the function (11.3); that is $\Lambda(v)=c v^{-\nu}$, where

$$
c=\frac{a V(x, R)^{\nu}}{R^{2}}
$$

Similarly to Example 10.2, we can apply Proposition 10.4 to the manifold $N=$ $B(x, R)$ and obtain, for any $y \in B(x, R)$,

$$
p_{N}(t, y, y) \leq \frac{\text { const }}{(c t)^{1 / \nu}}=\frac{\text { const }}{V(x, R)}\left(\frac{R^{2}}{t}\right)^{1 / \nu} .
$$

By taking $R=\sqrt{t}$ and $y=x$, we conclude

$$
p_{B(x, \sqrt{t})}(t, x, x) \leq \frac{\text { const }}{V(x, \sqrt{t})} .
$$

This estimate is clearly weaker than (11.5). One needs a more complicated argument as in [80, Proposition 5.2] to obtain such an estimate for the global heat kernel $p(t, x, x)$. 
11.2. Covering manifolds. Let $M$ and $K$ be Riemannian manifolds, $K$ being compact. A covering map $F: M \rightarrow K$ is regular if there is a discrete group $\Gamma$ of isometries of $M$ such that $F(x)=F(y)$ if and only if the points $x, y$ belong to the same orbit of $\Gamma$. Then we have $K \cong M / \Gamma$. The group $\Gamma$ is called a deck transformation group of $f$.

One says that $M$ is a regular cover of $K$ if a regular covering map $F: M \rightarrow K$ exists. A particular case of a regular cover is a universal cover for which $\Gamma=\pi_{1}(K)$.

Theorem 11.2. (Varopoulos [183], [188, Section X.3]) Let $M$ be a geodesically complete non-compact manifold which is a regular cover of a compact manifold $K$. Then the non-parabolicity of $M$ is equivalent to (11.1), that is

$$
\int^{\infty} \frac{r d r}{V(x, r)}<\infty
$$

for some/all $x \in M$.

Proof. The original proof of Varopoulos was rather involved and used algebraic structure theorems for groups. The present proof is much easier and is based on the following powerful isoperimetric inequality of Coulhon and Saloff-Coste.

Theorem 11.3. (Coulhon and Saloff-Coste [38, Theorem 4]) Let a geodesically complete non-compact manifold $M$ be a regular cover of a compact manifold $K$. Set for some fixed $x_{0}$

$$
V(r):=V\left(x_{0}, r\right) .
$$

Then, for some (large) constant $C>0$, the manifold $M$ satisfies the isoperimetric inequality (8.7) with the function

$$
f(v):=\frac{v}{C V^{-1}(C v)},
$$

where $V^{-1}$ is the inverse function.

Proof of Theorem 11.2. We need to show that the hypothesis (11.9) implies the non-parabolicity of $M$ (the other direction is covered by Theorem 7.3). By Theorem 8.2, $M$ is non-parabolic provided (8.8) holds. The integral in (8.8) is finitely proportional to

$$
\begin{aligned}
\int^{\infty} \frac{\left[V^{-1}(v)\right]^{2} d v}{v^{2}} & =\int^{\infty} \frac{r^{2} d V(r)}{V^{2}(r)} \\
& =-\int^{\infty} r^{2} d\left(\frac{1}{V(r)}\right) \\
& =-\left.\frac{r^{2}}{V(r)}\right|^{\infty}+2 \int^{\infty} \frac{r d r}{V(r)}
\end{aligned}
$$

which is finite by (11.9).

Corollary 11.4. (Varopoulos [187]) Let a geodesically complete non-compact manifold $M$ be a regular cover of a compact manifold $K$ with a deck transformation group $\Gamma$. Then $M$ is parabolic if and only if $\Gamma$ contains a finite index subgroup isomorphic with $\mathbb{Z}$ or $\mathbb{Z}^{2}$. 
Proof. Let $\gamma(n)$ be the volume growth function of $\Gamma$; i.e. $\gamma(n)$ is a cardinal number of a combinatorial ball of radius $n$ in $\Gamma$ (associated with some fixed set of generators). Then the behaviour of $\gamma(n)$ and $V(x, n)$ as $n \rightarrow \infty$ is the same so that the condition (11.9) is equivalent to

$$
\sum^{\infty} \frac{n}{\gamma(n)}<\infty
$$

It is a consequence of Theorems of Bass [9] and Gromov [89] that, for all $n \geq 1$, either $\gamma(n) \geq c n^{3}$ (and $M$ is non-parabolic) or $\gamma(n) \leq C n^{2}$ (and $M$ is parabolic). In the latter case, the group $\Gamma$ contains a finite index subgroup isomorphic with $\mathbb{Z}$ or $\mathbb{Z}^{2}$ (see [190, Corollary 3.18] for more details).

\section{Transience on manifolds with a pole}

In this section, we assume that $M$ is a geodesically complete manifold with a pole $o$ and prove a non-parabolicity test for such a manifold. As was mentioned in Section 3.1, the domain of the polar coordinates $(\rho, \theta)$ is $M \backslash\{o\}$, and the Riemannian metric of $M$ has the form (3.1), that is

$$
d s^{2}=d \rho^{2}+A_{i j}(\rho, \theta) d \theta^{i} d \theta^{j} .
$$

We assume in the sequel that $\theta^{1}, \theta^{2}, \ldots, \theta^{d-1}$ are the normal coordinates on $\mathbb{S}^{d-1}$. Denote $B_{\rho}=B(o, \rho)$. As (3.2) says, the area element on the geodesic sphere $\partial B_{\rho}$ is $\sqrt{\operatorname{det}\left\|A_{i j}\right\|}|d \theta|$ where $|\cdot|$ denotes here the standard volume on $\mathbb{S}^{d-1}$. Let us set

$$
D(\rho, \theta):=\sqrt{\operatorname{det}\left\|A_{i j}\right\|} .
$$

The following result is essentially due to Doyle [52], who derived it in the twodimensional case by using a nice heuristic argument. Here we provide a rigorous proof for any dimension, following [84].

Theorem 12.1. (Doyle [52]) If

$$
\int_{\mathbb{S}^{d-1}} \frac{|d \theta|}{\int_{1}^{\infty} D^{-1}(\rho, \theta) d \rho}>0
$$

then the manifold $M$ is non-parabolic.

The condition (12.3) can be rewritten as

$$
\text { meas }\left(\theta \in \mathbb{S}^{d-1}: \int^{\infty} \frac{d \rho}{D(\rho, \theta)}<\infty\right)>0 .
$$

For comparison, the sufficient condition for parabolicity (7.15) can also be expressed via $D$ as follows

$$
\int^{\infty} \frac{d \rho}{\int_{\mathbb{S}^{d-1}} D(\rho, \theta)|d \theta|}=\infty
$$

Let $\omega$ be a small region on $\mathbb{S}^{d-1}$. To satisfy (12.4), it suffices that $D(\rho, \theta)$ is growing in $\rho$ fast enough in a cone

$$
N^{\omega}:=\{(\rho, \theta): \theta \in \omega\},
$$

regardless of the values of $D$ outside the cone. This reflects the nature of the transience for which it suffices that the Brownian motion has some escape root to infinity, such as the cone $N^{\omega}$ (cf. Theorem 5.1(2) and Proposition 14.1(i)). On the 


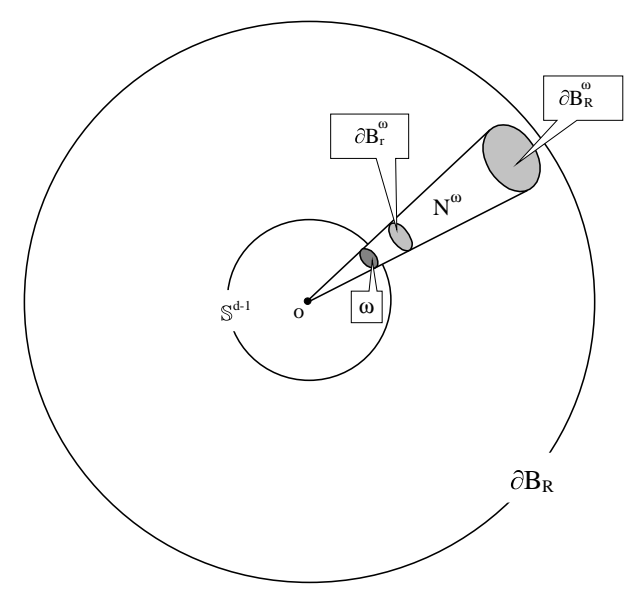

Figure 24. Capacitor $\left(B_{r}^{\omega}, B_{R}^{\omega}\right)$ in $N^{\omega}$

contrary, in order to satisfy $(12.5), D(\rho, \theta)$ should be growing relatively slowly in $\rho$ after being averaged in $\theta$.

The function $D$ has especially clear meaning if $d=2$, in which case the metric (12.1) is expressed directly via $D$ as follows

$$
d s^{2}=d \rho^{2}+D^{2}(\rho, \theta) d \theta^{2} .
$$

Proof of Theorem 12.1. It suffices to prove the following capacity estimate

$$
\operatorname{cap}\left(B_{r}, B_{R}\right) \geq \int_{\mathbb{S}^{d-1}} \frac{|d \theta|}{\int_{r}^{R} D^{-1}(\rho, \theta) d \rho},
$$

and the rest will follow by Theorem 5.1(6).

We start with the following two lemmas. Let $\omega$ be a region with a smooth boundary on $\mathbb{S}^{d-1}$. Consider the cone

$$
N^{\omega}=\left\{(\rho, \theta) \in \mathbb{R}^{d}: \rho>0 \text { and } \theta \in \bar{\omega}\right\},
$$

which is diffeomorphic to $\mathbb{R}_{+} \times \bar{\omega}$ and can be regarded as a manifold with the boundary $\partial N^{\omega}=\mathbb{R}_{+} \times \partial \omega$. Let us endow $N^{\omega}$ with the Riemannian metric (12.1) so that $N^{\omega}$ can be regarded as a geodesic cone in $M$ with the vertex $o$.

Denote by $B_{r}^{\omega}$ the "ball" $\left\{(\rho, \theta) \in N^{\omega}: \rho<r\right\}$ (see Figure 24).

Lemma 12.2. If, for $\rho \in[r, R]$, the function $D(\rho, \theta)$ does not depend on $\theta$, then, on the manifold $N^{\omega}$,

$$
\operatorname{cap}\left(B_{r}^{\omega}, B_{R}^{\omega}\right)=|\omega|\left(\int_{r}^{R} \frac{d \rho}{D(\rho)}\right)^{-1}
$$

Proof. The capacity $\operatorname{cap}\left(B_{r}^{\omega}, B_{R}^{\omega}\right)$ can be computed by (4.22), similarly to the case of a model manifold, if we take

$$
S(\rho):=|\omega| D(\rho),
$$


which is the boundary area of $\partial B_{\rho}^{\omega}$. Indeed, by (3.4), the radial part of the Laplace operator has in the region $r<\rho<R$ the form

$$
\Delta_{\text {radial }}=\frac{\partial^{2}}{\partial \rho}+\frac{D^{\prime}}{D} \frac{\partial}{\partial \rho}=\frac{\partial^{2}}{\partial \rho}+\frac{S^{\prime}}{S} \frac{\partial}{\partial \rho} .
$$

Therefore, the function $u(\rho)$ defined by (4.20), that is,

$$
u(\rho)=a \int_{\rho}^{R} \frac{d \xi}{S(\xi)}
$$

is harmonic in $B_{R}^{\omega} \backslash B_{r}^{\omega}$. In addition, $u$ satisfies the Neumann boundary condition $\left.\frac{\partial u}{\partial \nu}\right|_{\partial N \omega}=0$ because $u$ is a constant on the sphere $\partial B_{\rho}^{\omega}$ that is orthogonal to $\partial N^{\omega}$. Furthermore, if

$$
a:=\left(\int_{r}^{R} \frac{d \xi}{S(\xi)}\right)^{-1}
$$

then we have also $u(r)=1, u(R)=0$.

Hence, $u$ is the capacity potential of the capacitor $\left(B_{r}^{\omega}, B_{R}^{\omega}\right)$, whence

$$
\operatorname{cap}\left(B_{r}^{\omega}, B_{R}^{\omega}\right)=-\operatorname{flux}_{\partial B_{r}^{\omega}} u=\int_{\partial B_{r}^{\omega}} \frac{a}{S(r)} d \mu^{\prime}=a=|\omega|\left(\int_{r}^{R} \frac{d \xi}{D(\xi)}\right)^{-1},
$$

which was to be proved.

Lemma 12.3. Let $\hat{D}(\rho)$ be a smooth positive function on $[r, R]$ such that

$$
D(\rho, \theta) \geq \hat{D}(\rho), \quad \text { for all } \rho \in[r, R], \theta \in \omega .
$$

Then

$$
\operatorname{cap}\left(B_{r}^{\omega}, B_{R}^{\omega}\right) \geq|\omega|\left(\int_{r}^{R} \frac{d \rho}{\hat{D}(\rho)}\right)^{-1} .
$$

Proof. Denote by $\mathcal{L}^{\omega}(r, R)$ the set of test functions for the capacitor $\left(B_{r}^{\omega}, B_{R}^{\omega}\right)$ on $N^{\omega}$, that is all locally Lipschitz functions $\phi$ on $N^{\omega}$ such that $0 \leq \phi \leq 1,\left.\phi\right|_{B_{r}^{\omega}}=1$ and $\phi=0$ outside $B_{R}^{\omega}$. We have

$$
|\nabla \phi|^{2} \geq\left|\frac{\partial \phi}{\partial \rho}\right|^{2}
$$

and

$$
d \mu=D(\rho, \theta)|d \theta| d \rho
$$

whence

$$
\begin{aligned}
\operatorname{cap}\left(B_{r}^{\omega}, B_{R}^{\omega}\right) & =\inf _{\phi \in \mathcal{L}^{\omega}(r, R)} \int_{B_{R}^{\omega}}|\nabla \phi|^{2} d \mu \\
& \geq \inf _{\phi \in \mathcal{L}^{\omega}(r, R)} \int_{B_{R}^{\omega}}\left|\frac{\partial \phi}{\partial \rho}\right|^{2} d \mu \\
& =\inf _{\phi \in \mathcal{L}^{\omega}(r, R)} \int_{r}^{R} \int_{\omega}\left|\frac{\partial \phi}{\partial \rho}\right|^{2} D(\rho, \theta)|d \theta| d \rho \\
& \geq \inf _{\phi \in \mathcal{L}^{\omega}(r, R)} \int_{r}^{R} \int_{\omega}\left|\frac{\partial \phi}{\partial \rho}\right|^{2} \hat{D}(\rho)|d \theta| d \rho
\end{aligned}
$$


Let us consider another metric on $N^{\omega}$, which is given by

$$
d s^{2}=d \rho^{2}+\sigma^{2}(\rho) d \theta^{2},
$$

where $\sigma$ is a smooth positive function on $(0, \infty)$ such that $\sigma^{d-1}(\rho)=\hat{D}(\rho)$ for $\rho \in[r, R]$. Denote by $N_{\sigma}^{\omega}$ the cone $N^{\omega}$ with the metric (12.13). Then $\hat{D}(\rho)$ is the area density function in the region $r<\rho<R$ on $N_{\sigma}^{\omega}$.

It easy to see that the Euler-Lagrange equation for the functional (12.12) is

$$
\frac{\partial}{\partial \rho}\left(\hat{D}(\rho) \frac{\partial \phi}{\partial \rho}\right)=0
$$

Its solution $\phi$ with the boundary conditions $\phi(r, \theta)=1$ and $\phi(R, \theta)=0$ is clearly independent of $\theta$ and is equal to the capacity potential of the capacitor $\left(B_{r}^{\omega}, B_{R}^{\omega}\right)$ on the manifold $N_{\sigma}^{\omega}$ (cf. (12.9)). Hence, the infimum in (12.12) is equal to the capacity of $\left(B_{r}^{\omega}, B_{R}^{\omega}\right)$ on the manifold $N_{\sigma}^{\omega}$. This capacity was computed in Lemma 12.2 , whence (12.11) follows.

In order to prove (12.6), let us consider a finite family of small disjoint regions $\omega_{1}, \omega_{2}, \ldots$ on $\mathbb{S}^{d-1}$. Then we have

$$
\begin{aligned}
\operatorname{cap}\left(B_{r}, B_{R}\right) & =\inf _{\phi \in \mathcal{L}\left(B_{r}, B_{R}\right)} \int_{B_{R}}|\nabla \phi|^{2} d \mu \\
& \geq \inf _{\phi \in \mathcal{L}\left(B_{r}, B_{R}\right)} \sum_{i} \int_{B_{R} \cap N^{\omega_{i}}}|\nabla \phi|^{2} d \mu \\
& \geq \sum_{i} \inf _{\phi \in \mathcal{L} \omega(r, R)} \int_{B_{R}^{\omega_{i}}}|\nabla \phi|^{2} d \mu \\
& =\sum_{i} \operatorname{cap}_{N^{\omega_{i}}}\left(B_{r}^{\omega_{i}}, B_{R}^{\omega_{i}}\right) .
\end{aligned}
$$

Assuming that, for any $\omega_{i}$, we have chosen a function $\hat{D}_{i}(\rho)$ as in (12.10) and applying (12.11) to all $\omega_{i}$, we obtain

$$
\operatorname{cap}\left(B_{r}, B_{R}\right) \geq \sum_{i}\left|\omega_{i}\right|\left(\int_{r}^{R} \frac{d \rho}{\hat{D}_{i}(\rho)}\right)^{-1} .
$$

If $\omega_{i}$ is small enough, then $\hat{D}_{i}(\rho)$ can be arbitrarily close to $D(\rho, \theta)$, uniformly in $\theta \in \omega_{i}$ and $\rho \in[r, R]$. Also, the sum on the right-hand side of (12.14) can be replaced by integration as we refine the partition of $\mathbb{S}^{d-1}$ into $\omega_{i}$. Therefore, the sum in (12.14) can be made arbitrarily close to

$$
\int_{\mathbb{S}^{d-1}}\left(\int_{r}^{R} \frac{d \rho}{D(\rho, \theta)}\right)^{-1}|d \theta|,
$$

whence (12.6) follows.

\section{LIOUVILLE PROPERTIES}

The classical Liouville theorem says that any bounded harmonic function in $\mathbb{R}^{d}$ is identically constant. We will consider here various generalizations of this property. Given a function class $\mathcal{F}$ on $M$ and an elliptic operator $L$, by the Liouville property of the pair $(\mathcal{F}, L)$ we mean an assertion that any function in $\mathcal{F}$ solving the equation $L u=0$ is a constant. Examples of operators are the Laplace operator 
or a Schrödinger operator; examples of function classes are $L^{p}(M, \mu)$, non-negative functions, etc.

There is a vast literature devoted to Liouville type theorems and their generalizations. We have selected only those Liouville properties which relate to recurrence or non-explosion properties.

13.1. $L^{p}$-sub(super)harmonic functions. Let $u(x)$ be a non-negative $C^{2}$ function on $M$. Fix a point $x_{0} \in M$ and denote for $r>0$ and $p \in \mathbb{R}$

$$
v_{p}(r):=\int_{B\left(x_{0}, r\right)} u^{p}(x) d \mu(x) .
$$

One may wonder what growth of $v_{p}(r)$ as $r \rightarrow \infty$ may occur provided $u(x)$ is a harmonic function. A partial answer is given by the following theorem:

Theorem 13.1. (Sturm [173, Theorem 1]) Assume that $M$ is geodesically complete and that

(a) either $p \in(-\infty, 1)$ and $u \geq 0$ is superharmonic

(b) or $p \in(1, \infty)$ and $u \geq 0$ is subharmonic.

Then the condition

$$
\int^{\infty} \frac{r d r}{v_{p}(r)}=\infty
$$

implies that $u(x) \equiv$ const.

It is not accidental that (13.1) looks similar to the parabolicity condition (7.12). Indeed, let us assume (7.12) and deduce from Theorem 13.1 that $M$ is parabolic. Let $u$ be any bounded positive superharmonic function on $M$. Obviously, for this function and for $p=0$ we have $v_{p}(r) \leq$ const $V\left(x_{0}, r\right)$, and the hypothesis (13.1) is implied by (7.12). Theorem 13.1 says that $u \equiv$ const. By Theorem 5.1(3), we conclude that $M$ is parabolic.

Thus, the case $p=0$ corresponds to parabolicity. The case $p>1$ is related to a $L^{p}$-Liouville theorem of Yau [193] which says that any non-negative subharmonic function $u \in L^{p}(M, \mu)$ is necessarily constant (see [120] for a detailed discussion about $L^{p}$-harmonic functions). This is also contained in Theorem 13.1 because $u \in L^{p}(M, \mu)$ implies boundedness of $v_{p}(r)$ and thus (13.1).

It turns out that stochastic completeness is also somewhat related to the statement of Theorem 13.1: it corresponds to the case $p=1$ which is not covered by this theorem, though. It is known [31], [120], [75] that there exists a geodesically complete manifold which carries a non-constant positive harmonic function $u \in L^{1}(M, \mu)$. However, such a manifold is necessarily stochastically incomplete, because of the following theorem.

Theorem 13.2. ([75, Theorem 3]) If $M$ is stochastically complete, then any nonnegative superharmonic function $u \in L^{1}(M, \mu)$ is a constant.

Proof. If $M$ is parabolic, then $u$ is constant by definition. Assume that $M$ is nonparabolic and prove that $u \equiv 0$. If $u \neq \equiv 0$ then $u$ is strictly positive, by the strong minimum principle. Take any precompact open set $U \in M$, a point $y \in U$ and find a large constant $C$ such that, for all $x \in \partial U$,

$$
C u(x) \geq G(x, y) .
$$


The superharmonicity of $u$ and the minimum principle imply that (13.2) holds for all $x \in M \backslash U$ (one should verify it first for $G_{\mathcal{E}_{k}}$ and then pass to the limit as $k \rightarrow \infty$ - see Section 4.2). Hence, $G(\cdot, y) \in L^{1}(M \backslash U, \mu)$ and $M$ is stochastically incomplete by Corollary 6.7.

The following theorem fills the gap between $L^{1}$ and $L^{p}, p>1$.

Theorem 13.3. (Nadirashvili [145]) Assume that $M$ is geodesically complete and $u \geq 0$ is a subharmonic function satisfying the condition

$$
\int_{M} \frac{f(u(x)) d \mu(x)}{1+\rho^{2}(x)}<\infty
$$

where $\rho(x):=\operatorname{dist}\left(x, x_{0}\right)$ with a fixed $x_{0} \in M$ and $f(\cdot) \geq 0$ is a strictly monotonically increasing function on $[0, \infty)$ such that

$$
\int^{\infty} \frac{d t}{f(t)}<\infty
$$

Then $u \equiv$ const.

The condition (13.4) is satisfied, for example, by $f(t)=t^{p}, p>1$ and $f(t)=$ $t \log t(\log \log t)^{p}$ (the latter is contained implicitly also in [120, p.291]). Theorem 13.3 implies that if, for all large $r$ and some $\varepsilon>0$,

$$
\int_{B\left(x_{0}, r\right)} f(u(x)) d \mu(x) \leq C r^{2-\varepsilon},
$$

then $u \equiv$ const. It would be interesting to relax the hypothesis (13.5) to match (13.1) for $f(t)=t^{p}$.

Note that (13.4) is sharp in the following sense: if $f(t)$ does not satisfy (13.4), then there is a manifold $M$ (in fact, a model manifold) and a positive subharmonic function $u$ on $M$ such that

$$
\int_{M} f(u(x)) d \mu(x)<\infty .
$$

It would also be interesting to see if the case (a) of Theorem 13.1 can be extended in a similar way to fill the gap between $L^{p}, p<1$ and $L^{1}$.

See [120], [117], [119] for further results on $L^{p}$-subharmonic functions on manifolds.

13.2. Liouville property for Schrödinger equation. Let us consider on $M$ the stationary Schrödinger equation

$$
\Delta u-q(x) u=0,
$$

where $q(x) \geq 0, q \not \equiv 0$ is a continuous function on $M$, and $u \in C^{2}(M)$. We say that the function $u$ is $q$-harmonic if it satisfies (13.6). Similar to the definition of superand subharmonic functions, one introduces $q$-superharmonic and $q$-subharmonic functions (see Section 4.1). If $u \in C^{2}$ then $u$ is $q$-superharmonic if $\Delta u-q u \leq 0$ and $q$-subharmonic if $\Delta u-q u \geq 0$.

The question to be discussed here is whether there is a non-zero bounded $q$ harmonic function ${ }^{11}$ on $M$. Let us say that $M$ has the $q$-Liouville property if the only bounded $q$-harmonic function is 0 . The connection of the $q$-Liouville property

\footnotetext{
${ }^{11}$ Let us emphasize that no constant function is $q$-harmonic except for zero, due to $q \not \equiv 0$.
} 
to recurrence and non-explosion follows from Theorems 5.1(7) and 6.2(3), which say that

- if $q \in C_{0}^{\infty}(M)$ then the $q$-Liouville property is equivalent to the parabolicity of $M$;

- if $q \equiv$ const $>0$ then the $q$-Liouville property is equivalent to the stochastic completeness of $M$.

There are the following relations between the $q$-Liouville properties with different $q$.

Theorem 13.4. ([88], [85])

(a) If the $q_{1}$-Liouville property holds and if $q_{2} \geq c q_{1}$ for some positive constant $c$, then the $q_{2}$-Liouville property is true as well.

(b) If the set $\left\{q_{1} \neq q_{2}\right\}$ is precompact, then the $q_{1}$-Liouville property is equivalent to the $q_{2}$-Liouville property.

In particular, parabolicity implies stochastic completeness, which we know otherwise by Corollary 6.4 .

Some tests for recurrence and stochastic completeness appear to be particular cases of more general statements about the $q$-Liouville property.

Definition 13.1. An open set $\Omega \subset M$ is called q-massive if there is at least one admissible $q$-subharmonic function for $\Omega$, that is, a non-negative bounded $q$ subharmonic function $v$ on $M$ such that $v=0$ in $M \backslash \Omega$ and $\sup _{\Omega} v>0$ (cf. Sections 6 and 4.4).

Similarly to Proposition 6.1, the $q$-massiveness is preserved by increasing a set and by reducing it by a compact. The following is a useful sufficient condition for $q$-non-massiveness generalizing Corollaries 5.4 and 6.6.

Proposition 13.5. Let $\Omega$ be an open set in $M$. Assume that there exists in $\Omega$ a non-negative q-superharmonic function $v$ such that $v(x) \rightarrow \infty$ as $x \rightarrow \infty$. Then $\Omega$ is not q-massive.

In the following theorem, we have collected various conditions for the absence of the $q$-Liouville property.

Theorem 13.6. The following statements are equivalent:

(a) There exists a non-zero bounded q-harmonic function on $M$.

(b) There exists a positive q-harmonic function on $M$.

(c) Manifold $M$ is q-massive (cf. Theorems 5.1 and 6.2).

(d) ([10], [85]) There exists a massive (not $q$-massive!) set $\Omega$ and a point $x_{0} \in M$ such that

$$
\int_{\Omega} G\left(x_{0}, x\right) q(x) d \mu(x)<\infty .
$$

Corollary 13.7. Let $\Omega$ be an exterior of a compact set in $M$. Assume that there exists in $\Omega$ a non-negative q-superharmonic function $v$ such that $v(x) \rightarrow \infty$ as $x \rightarrow \infty$. Then the $q$-Liouville property holds for $M$.

Indeed, $\Omega$ is not $q$-massive by Proposition 13.5. Hence, $M$ is not $q$-massive either, and the $q$-Liouville property holds by Theorem 13.6. 
Corollary 13.8. ([76]) If, for some point $x_{0} \in M$,

$$
\int_{M} G\left(x_{0}, x\right) q(x) d \mu(x)<\infty
$$

then there exists a non-zero bounded $q$-harmonic function on $M$; i.e. the $q$-Liouville property fails.

If $q \in C_{0}^{\infty}$ then (13.8) is equivalent to the finiteness of the Green function (=nonparabolicity) because the Green function on a manifold is always locally summable. If $q \equiv$ const $>0$ then (13.8) is equivalent to the global summability of the Green function which implies by Corollary 6.7 the stochastic incompleteness of $M$.

The following theorem unifies and generalizes the volume growth conditions (7.12) and (9.1) for parabolicity and stochastic completeness.

Theorem 13.9. ([76]) Let $M$ be geodesically complete and let us denote, for some point $x_{0} \in M$,

$$
Q(r):=\int_{0}^{r} \sqrt{\inf _{x \in \partial B\left(x_{0}, s\right)} q(x)} d s .
$$

Assume that, for all $r$ large enough and some $C>0$,

$$
V\left(x_{0}, r\right) \leq C r^{2} \exp \left(C Q^{2}(r / 2)\right) .
$$

Then the q-Liouville property holds on $M$.

For example, if $q \in C_{0}^{\infty}(M)$ then $Q$ is uniformly bounded, and (13.9) becomes

$$
V\left(x_{0}, r\right) \leq C r^{2} \text {. }
$$

Therefore, the fact that (13.10) implies parabolicity (see Theorem 7.3) is contained in Theorem 13.9. If $q \equiv 1$ then $Q(r)=r$ and (13.9) is equivalent to

$$
V\left(x_{0}, r\right) \leq \exp \left(C r^{2}\right) .
$$

Thus, we obtain again that (13.11) implies stochastic completeness (cf. Theorem 9.1).

Example 13.1. Suppose that $M=\mathbb{R}^{d}, d>2$, and $q$ is a decreasing function of $r=|x|$, at least for large $|x|$. Let $o$ be the origin in $\mathbb{R}^{d}$. Since $G(o, x)=\operatorname{const} r^{2-d}$ and $V(0, r)=$ const $r^{d-1}$, Corollary 13.8 implies that the $q$-Liouville property fails to hold provided

$$
\int^{\infty} q(r) r d r<\infty
$$

On the other hand, Theorem 13.9 implies that the $q$-Liouville property is true provided

$$
\int_{1}^{r} \sqrt{q(r)} d r \geq \operatorname{const} \sqrt{\log r}
$$

for $r$ large enough. For example, if $q(r)=\frac{1}{r^{2} \log ^{\alpha} r}$, then (13.12) is satisfied if $\alpha>1$, whereas (13.13) holds if $\alpha \leq 1$.

It is possible to prove in this case that if (13.12) does not hold, then the $q$ Liouville property is true - see [76, Corollary 3.1]. There is also a generalization of the hypothesis (13.9) to match (7.12) and (9.1). 


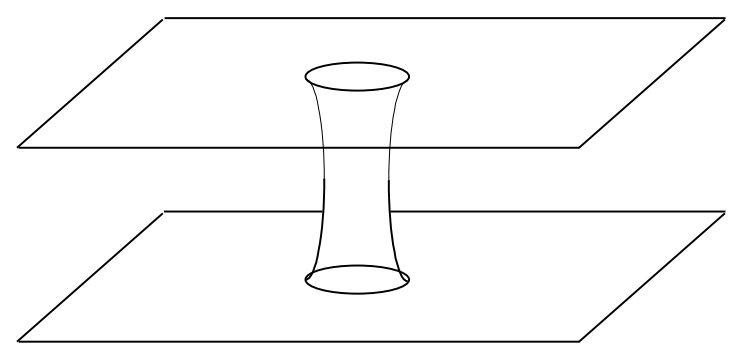

Figure 25. Connected sum of two copies of $\mathbb{R}^{d}$

13.3. Bounded harmonic functions. We briefly discuss the relation between the $L^{\infty}$-Liouville property and massive sets. By the $L^{\infty}$-Liouville property, we mean an assertion that any bounded harmonic function on $M$ is identically constant. It holds, for example, in any $\mathbb{R}^{d}$, and fails in $\mathbb{H}^{d}$. Another example of a geodesically complete manifold, which carries a non-trivial bounded harmonic function, is obtained by gluing together two copies of $\mathbb{R}^{d}, d>2$, by a compact pipe as on Figure 25 (see [115]).

Let us denote by $\mathcal{H}(M)$ the space of all bounded harmonic functions on $M$. The the $L^{\infty}$-Liouville property is equivalent to the fact that $\operatorname{dim} \mathcal{H}(M)=1$. For comparison, if we denote by $\mathcal{H}_{q}(M)$ the space of all bounded solutions to the Schrödinger equation (13.6), then the $q$-Liouville property means that $\operatorname{dim} \mathcal{H}_{q}(M)=0$.

Despite many works devoted to the $L^{\infty}$-Liouville property, the major question of its geometric background is still open. Here we cite only one of the results related to our topic (see [119] for a thorough account of the $L^{\infty}$-Liouville property on manifolds as well as [104] for the $L^{\infty}$-Liouville property on groups).

The following theorem shows that both parabolicity and the $L^{\infty}$-Liouville property are controlled by a number of non-intersecting proper massive sets on $M$.

Theorem 13.10. (a) (Myrberg [144], Royden [163, Proposition 23]) The nonparabolicity of $M$ is equivalent to existence of a proper massive set on $M$ (cf. Theorem 5.1(2)).

(b) (Nevanlinna [151], Bader-Parreau [7], Mori [141], Royden [163, Proposition 24]) The negation of the $L^{\infty}$-Liouville property (=existence of a non-trivial bounded harmonic function) is equivalent to the existence of two disjoint massive sets on $M .^{12}$

(c) ([77]) $\operatorname{dim} \mathcal{H}(M)$ is equal to the supremum of the number of disjoint massive sets which can be put on $M .{ }^{13}$

For example, for the manifold on Figure 25, one has $\operatorname{dim} \mathcal{H}(M)=2$ (provided $d>2$ ) because each of two sheets is massive, and none of them contains more than one massive set (see [115]).

For further applications of the notion of massiveness see [73], [94], [125].

\footnotetext{
${ }^{12}$ Similarly, existence of a non-trivial harmonic function with a finite Dirichlet integral is equivalent to the existence of two disjoint $D$-massive sets.

${ }^{13}$ Similarly, the dimension of the space of bounded harmonic functions with a finite Dirichlet integral is equal to the maximum number of disjoint $D$-massive sets. The dimension here may be finite or infinite. In the latter case, we do not distinguish between different sorts of infinite cardinalities.
} 
13.4. Minimal surfaces. The celebrated theorem of Bernstein says that any minimal surface $M$ in $\mathbb{R}^{3}$, which is a graph of a function in the whole $\mathbb{R}^{2}$, is necessarily a plane. In other words, if

$$
M:=\left\{(x, y, z) \in \mathbb{R}^{3}: z=f(x, y),-\infty<x<\infty,-\infty<y<\infty\right\}, \quad f \in C^{\infty}
$$

and if $M$ is a minimal surface, then $f$ is an affine function. L.Bers [13] and R.Finn [62] observed that this statement is closely related to parabolicity of $M$ as a manifold. We sketch here the proof of Bernstein's theorem following this approach.

The proof contains two essential ingredients.

(i) The observation that the function $v=\arctan f_{x}$ (as well $\operatorname{as} \arctan f_{y}$ ) satisfies the equation $\Delta v=0$ on $M$.

(ii) The claim that $M$ is parabolic.

As soon as we have (i) and (ii), we argue as follows. The function $v$ is a bounded harmonic function on $M$. Since $M$ is parabolic, $v$ should be a constant, by Theorem 5.1(3). Therefore, $f_{x}$ and $f_{y}$ are constants, and $f$ is affine.

The fact that $\arctan f_{x}$ is harmonic on $M$ was observed by S.Bernstein [12]. The proof can be found in [155, p.237].

Let us prove that $M$ is parabolic. The Riemannian metric on $M$ is inherited from $\mathbb{R}^{3}$. Therefore, any geodesic ball $B(x, R)$ on $M$ lies in the Euclidean ball $B_{e}(x, R) \subset \mathbb{R}^{3}$. It is known that a minimal surface, which is a graph, is also an area minimizer. Thus, the area of $M \cap B_{e}(x, R)$ is bounded from above by the area of $\partial B_{e}(x, R)$. Therefore, we obtain $V(x, R) \leq 4 \pi R^{2}$. By Corollary 7.4, $M$ is parabolic.

Further relations between Bernstein's type theorems and Liouville theorems can be found in [17], [28], [138], [168]. See [157] for a general overview of minimal surfaces.

13.5. Liouville property on Riemannian products. Let $N$ be a Riemannian manifold satisfying the $L^{\infty}$-Liouville property; i.e. any bounded harmonic function on $N$ is a constant. Let us ask the question whether the Riemannian product $N \times K$ possesses the same property, where $K$ is a compact Riemannian manifold. Surprisingly enough, the answer is in general no. A counterexample which will be described below is based on an example of a manifold $N$ constructed by Pinchover [160], such that

(i) $N$ is geodesically complete;

(ii) $N$ satisfies the $L^{\infty}$-Liouville property;

(iii) $N$ is stochastically incomplete.

In fact, the manifold of Pinchover has also $\lambda_{1}(N)=0$, but we will not use this.

Given a manifold $N$ with properties (i)-(iii), let us show that $N \times K$ possesses a non-constant bounded harmonic function. The Laplace operator $\Delta_{K}$ in $L^{2}(K)$ has a discrete spectrum. Let $\lambda>0$ be one of the eigenvalues with the eigenfunction $w$ so that

$$
\Delta_{K} w+\lambda w=0 .
$$

Theorem 6.2 says that there is a bounded non-constant $\lambda$-harmonic function $v$ on the stochastically incomplete manifolds $N$, that is,

$$
\Delta_{N} v-\lambda v=0 .
$$




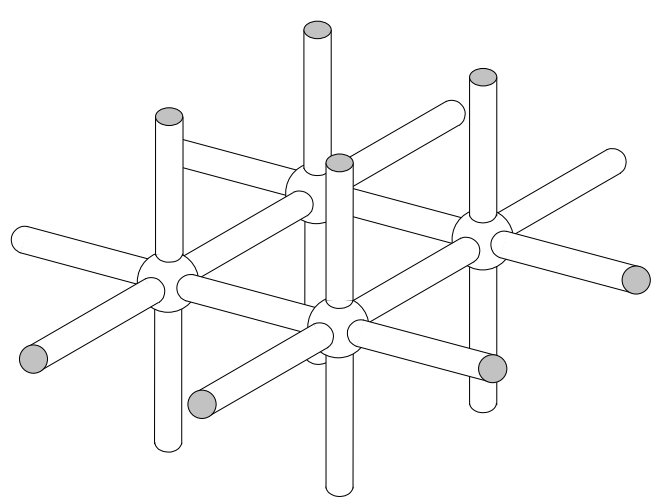

Figure 26. The jungle gym in $\mathbb{R}^{3}$

Therefore, the function $u=v \otimes w$ on $N \times K$ satisfies the Laplace equation

$$
\Delta_{N \times K} u=\left(\Delta_{N}+\Delta_{K}\right) u=0,
$$

is bounded and non-constant.

This argument can be extended so as to show that $N \times K$ possesses the $L^{\infty}$ Liouville property if and only if $N$ possesses the $L^{\infty}$-Liouville property and $N$ is stochastically complete.

Now we describe briefly the manifold of Pinchover with properties (i)-(iii). Let $M$ be a geodesically complete manifold such that

(a) for a fixed point $o \in M$ and for all large enough $R$

$$
V(o, R) \leq C R^{3}
$$

(b) for large enough $r:=\operatorname{dist}(x, o)$

$$
G(x, o) \leq \frac{C}{r}
$$

(c) $M$ possesses the $L^{\infty}$-Liouville property;

(d) $\operatorname{dim} M=2$.

All (a)-(c) are valid if $M=\mathbb{R}^{3}$, but we need $M$ to be two dimensional. One of the ways of constructing such a manifold is to take $M$ as a blown-up jungle gym in $\mathbb{R}^{3}$ (see Figure 26) which is roughly isometric ${ }^{14}$ to $\mathbb{R}^{3}$ and thus possesses (a)-(d).

Denote by $g$ the Riemannian metric of $M$. Given a positive smooth function $\rho(x)$, let us introduce a conformal metric $\widehat{g}=\rho^{2} g$ and let $N:=(M, \widehat{g})$. Let us denote by $\widehat{\Delta}, \widehat{G}, \widehat{\mu}$ the Laplace operator, the Green function and the Riemannian measure on $N$ respectively. If $\operatorname{dim} N=d$ then we have

$$
\widehat{\mu}=\rho^{d} \mu \quad \text { and } \quad \widehat{\Delta}=\rho^{-d} \operatorname{div}\left(\rho^{d-2} \nabla\right)
$$

which implies, for $d=2$,

$$
\widehat{\mu}=\rho^{2} \mu \text { and } \widehat{\Delta}=\rho^{-2} \Delta .
$$

In particular, harmonic functions on $M$ and $N$ coincide, whence $N$ satisfies the $L^{\infty}$-Liouville property. The relations (13.16) imply for the Green kernel

$$
\widehat{G}=G \text {. }
$$

\footnotetext{
${ }^{14}$ See the papers of Kanai [106] and [107] for the notion and the properties of a rough isometry.
} 
Let us show how to choose $\rho$ to ensure geodesic completeness and stochastic incompleteness of $N$. The function $\rho(x)$ will depend only on $r=\operatorname{dist}(x, o)$ so that we will write also $\rho(r)$ (if $r$ is not smooth enough, then one can use a smooth approximation afterwards). Geodesic completeness of $N$ will follow from

$$
\int^{\infty} \rho(r) d r=\infty
$$

(which implies that the length of a geodesic ray on $N$ is $\infty$ ). Stochastic incompleteness of $N$ will follow, by Corollary 6.7, from

$$
\int_{N} \widehat{G}(o, x) d \widehat{\mu}(x)<\infty
$$

which, by (13.17) and (13.16), is equivalent to

$$
\int_{M} G(o, x) \rho^{2}(x) d \mu(x)<\infty .
$$

Due to the estimate (13.15), this amounts to

$$
\int^{\infty} \frac{\rho^{2}(r)}{r} d V(o, r)<\infty .
$$

We are left to choose $\rho(r)$ to satisfy (13.18) and (13.21). Let us set, for $r$ large enough,

$$
\rho(r)=\frac{1}{r \log r} .
$$

Then (13.18) is obvious, and (13.21) follows by integration-by-parts from (13.14) .

A minor modification of the above argument is required so as to have also $\lambda_{1}(N)=0$. Now we assume in addition that (13.14) holds for any point $o$ (which is the case for the jungle gym). The function $\rho$ will no longer be radial. We set, instead of (13.22),

$$
\rho(x)=\rho_{0}(r)+\delta(x),
$$

where $r=\operatorname{dist}(x, o)$, the function $\rho_{0}(r)$ is defined by (13.22) and the function $\delta(x)$ is defined as follows. Choose a sequence of points $x_{i} \in M, i=1,2,3, \ldots$ so that $\operatorname{dist}\left(x_{i}, o\right)=4^{i}$, and let

$$
\delta(x) \begin{cases}=1, & x \in B\left(x_{i}, i\right) \\ \in[0,1], & x \in B\left(x_{i}, 2 i\right) \backslash B\left(x_{i}, i\right) \\ =0, & \text { otherwise }\end{cases}
$$

assuming that $\delta \in C^{\infty}$. Then (13.20) holds again because, by (13.14) and (13.15),

$$
\int_{M} G(o, x) \delta^{2}(x) d \mu(x) \leq \mathrm{const} \sum_{i} \frac{V\left(x_{i}, 2 i\right)}{4^{i}} \leq \mathrm{const} \sum_{i} \frac{i^{3}}{4^{i}}<\infty .
$$

Finally, we have, for any $i$,

$$
\lambda_{1}(N) \leq \lambda_{1}\left(B\left(x_{i}, i\right), \widehat{g}\right) \leq \mathrm{const} \lambda_{1}\left(B\left(x_{i}, i\right), g\right) \leq \frac{\mathrm{const}}{i^{2}}
$$

(because the metric $\widehat{g}$ is nearly Euclidean in $B\left(x_{i}, i\right)$ ), whence $\lambda_{1}(N)=0$. 


\section{HARMONIC FUNCTIONS ON MANIFOLDS WITH ENDS}

Let $M$ be a geodesically complete non-compact manifold. We say that an open set $E \subset M$ is an end if it is connected, unbounded and if its boundary $\partial E$ is compact. When necessary, we will assume that $\partial E$ is smooth enough. We say that $M$ is a manifold with ends if $M$ is a union of a compact set and a finite number of disjoint ends (see Figure 27).

The purpose of this section is to describe the sets of bounded and positive harmonic functions on a manifold with ends, assuming that we have enough information about the ends. As it turns out, the answer depends on the property of an end being parabolic or not.

14.1. Parabolic subsets and ends. Let us define the notion of parabolicity of an open subset $E \subset M$. The motivation behind this definition is a desire to treat the closure $\bar{E}$ as a manifold with a boundary and to apply to $\bar{E}$ the notion of parabolicity of a manifold, assuming the Neumann boundary condition on $\partial E$ (see the remark after Theorem 5.1). For an arbitrary open subset, it is convenient to use the capacity definition of parabolicity since it requires no smoothness of the boundary. Hence, we say that an open subset $E \subset M$ is parabolic if, for any compact $K \subset M$,

$$
\operatorname{cap}_{E}(K)=0
$$

(see Section 4.3 for the definition of capacity). Clearly, if $M$ is a parabolic manifold, then $M$ is parabolic also as a subset.

In the next statement, we collect simple properties of parabolic subsets and ends.

Proposition 14.1. Let $E$ and $E^{\prime}$ be open subsets of a manifold $M$.

(a) If $E \subset E^{\prime}$ and if $E^{\prime}$ is parabolic, then $E$ is parabolic, too. In particular, any subset of a parabolic manifold $M$ is a parabolic set.

(b) If $E \backslash E^{\prime}$ is precompact and if $E^{\prime}$ is parabolic, then $E$ is parabolic, too.

(c) If $\partial E$ is smooth, then the parabolicity of $E$ coincides with the parabolicity of $\bar{E}$ as a manifold with boundary.

(d) If $E$ is a proper massive set, then $E$ is non-parabolic. If $E$ is an end of $M$ and $E$ is non-parabolic, then $E$ is massive.

(e) If an exterior of a compact of $M$ consists of a disjoint union of a finite number of parabolic sets, then $M$ is parabolic. In particular, if $M$ is a manifold with ends, then its parabolicity is equivalent to parabolicity of all ends.



FIGURE 27. Manifold with ends 
Proof. (a) This follows from $\operatorname{cap}_{E}(K) \leq \operatorname{cap}_{E^{\prime}}(K)$, which is a consequence of the definition (4.18).

(b) By (a), we may assume that $E^{\prime} \subset E$. Let $K$ be a big enough compact that contains $E \backslash E^{\prime}$. Then the exterior of $K$ in $E$ and $E^{\prime}$ coincide, whence $\operatorname{cap}_{E}(K)=$ $\operatorname{cap}_{E^{\prime}}(K)=0$ and $E$ is parabolic.

(c) This follows from (4.19).

(d) If $E$ is a proper massive set, then, as follows from Proposition 4.3(ii), there exists a massive set $E^{\prime} \subset E$ with smooth boundary. The subharmonic potential $b_{E^{\prime}}$ is a non-constant bounded subharmonic function on the manifold with boundary $\overline{E^{\prime}}$. By Theorem 5.1(3), $\overline{E^{\prime}}$ is a non-parabolic manifold; by assertion (c), $E^{\prime}$ is a non-parabolic set; finally, by assertion (a), $E$ is also non-parabolic.

Now let $E$ be a non-parabolic end. Reducing $E$ by a compact, we may assume that $\partial E$ is smooth (the non-parabolicity of $E$ does not change). By assertion (c), $\bar{E}$ is a parabolic manifold and, by Theorem $5.1(2 \mathrm{a})$, an exterior of any compact in $\bar{E}$ is massive. Therefore, $E=\bar{E} \backslash \partial E$ is massive, both in $\bar{E}$ and $M$.

In general, a non-parabolic set may be non-massive, as a half-space of $\mathbb{R}^{d}, d>2$.

(e) Let $E_{1}, E_{2}, \ldots, E_{n}$ be such sets in $M$. If $K$ is a big enough compact in $M$, then, obviously,

$$
\operatorname{cap}_{M}(K)=\sum_{i} \operatorname{cap}_{E_{i}}(K),
$$

whence the statement follows.

We state the following result as an example of application of parabolic subsets.

Theorem 14.2. ([73, Theorem 1]) An open set $\Omega \subset M$ is $D$-massive if and only if there is a non-parabolic open set $E$, such that $\bar{E} \subset \Omega$ and $\operatorname{cap}(\bar{E}, \Omega)<\infty$.

As a consequence we see that the $D$-massiveness of $\Omega$ is invariant under a quasiisometry (cf. Corollary 5.3).

14.2. Spaces of harmonic functions on manifolds with ends. We denote by $\mathcal{H}(M)$ (resp. $\mathcal{H}_{+}(M)$ ) the set of all bounded (resp. positive) harmonic functions on $M$. The former is a linear space whereas the latter is a cone. We are interested in the dimensions of these spaces.

If $E$ is an open subset of $M$ with smooth boundary, then we define $\mathcal{H}(E)$ and $\mathcal{H}_{+}(E)$ similarly, with the additional assumption that the harmonic functions vanish on $\partial E$.

Theorem 14.3. (Sung, Tam, Wang [175, Theorem 3.2]) Let $M$ be a complete manifold with ends. Assume that $M$ has $s \geq 0$ parabolic ends $P_{1}, P_{2}, \ldots, P_{s}$ and $l \geq 1$ non-parabolic ends $N_{1}, N_{2}, \ldots, N_{l}$. Then

$$
\operatorname{dim} \mathcal{H}(M)=\sum_{j=1}^{l} \operatorname{dim} \mathcal{H}\left(N_{j}\right)
$$

and

$$
\operatorname{dim} \mathcal{H}_{+}(M)=\sum_{i=1}^{s} \operatorname{dim} \mathcal{H}_{+}\left(P_{i}\right)+\sum_{j=1}^{l} \operatorname{dim} \mathcal{H}_{+}\left(N_{j}\right)
$$


Remark. The hypothesis $l \geq 1$ is equivalent to non-parabolicity of the manifold $M$ itself, by Proposition 14.1(e). If $l=0$ and thus $M$ is parabolic, then there are no non-negative harmonic functions on $M$ except constants, and $\operatorname{dim} \mathcal{H}(M)=$ $\operatorname{dim} \mathcal{H}_{+}(M)=1$, regardless of the number of ends.

It is remarkable that the parabolic ends do not contribute to $\operatorname{dim} \mathcal{H}(M)$. One of the ways to understand that is to observe that for any parabolic end $P$ we have $\operatorname{dim} \mathcal{H}(P)=0$. Indeed, if $u$ is a bounded harmonic function $P$ vanishing on $\partial P$, then the positive part $u_{+}$is an admissible subharmonic function for $P$. However, the parabolicity of $P$ implies its non-massiveness, whence $u_{+} \equiv 0$. Similarly, $u_{-} \equiv 0$ and $u \equiv 0$.

Remark. If $M$ is a manifold with boundary, then $\mathcal{H}(M)$ (resp. $\left.\mathcal{H}_{+}(M)\right)$ denotes the space of bounded (resp. positive) harmonic functions on $M$ with the Neumann boundary condition on $\partial M$. For any end $E$, let us regard its closure $\bar{E}$ as a manifold with boundary and consider the spaces $\mathcal{H}(\bar{E})$ and $\mathcal{H}_{+}(\bar{E})$. We claim that, for any non-parabolic end $N$,

$$
\operatorname{dim} \mathcal{H}_{+}(N)=\operatorname{dim} \mathcal{H}_{+}(\bar{N})
$$

and

$$
\operatorname{dim} \mathcal{H}(N)=\operatorname{dim} \mathcal{H}(\bar{N})
$$

(this follows from [175, Theorem 2.6(a) and Proposition 2.7(a)]). For a parabolic end $P$, we have

$$
\operatorname{dim} \mathcal{H}(P)=0<1=\operatorname{dim} \mathcal{H}(\bar{P})=\operatorname{dim} \mathcal{H}_{+}(\bar{P}) \leq \operatorname{dim} \mathcal{H}_{+}(P)
$$

(see [175, Lemma 2.4]).

Corollary 14.4. ([123, Theorem 2.1]) Under the hypotheses of Theorem 14.3, we have

$$
\operatorname{dim} \mathcal{H}(M) \geq l
$$

and

$$
\mathcal{H}_{+}(M) \geq s+l .
$$

The estimate (14.4) follows also from Theorem 13.10(c). Indeed, $l$ non-parabolic ends provide $l$ disjoint massive sets on $M$ whence (14.4).

In the next section, we impose an additional hypothesis of regularity of an end which will ensure that the end does not split further into two smaller massive subsets.

14.3. Manifolds with regular ends. Given an end $E$, we denote, for any $r \geq 0$,

$$
E_{r}:=\{x \in E: \operatorname{dist}(x, \partial E)=r\} .
$$

We say that an end $E$ is regular if, for all $r$ large enough and for any positive harmonic function $u$ defined in

$$
U_{r}:=\left\{x \in E: \frac{r}{2}<\operatorname{dist}(x, \partial E)<2 r\right\}
$$

(see Figure 28) we have a Harnack type inequality on $E_{r}$

$$
\sup _{E_{r}} u \leq C \inf _{E_{r}} u,
$$




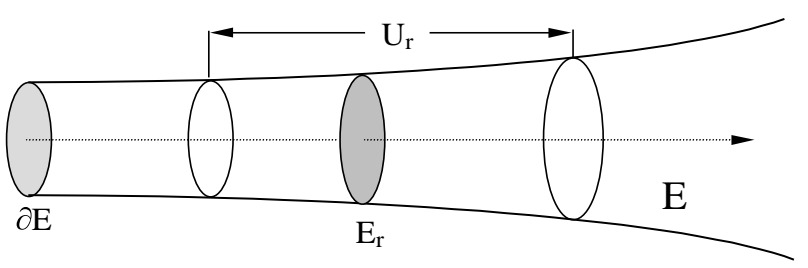

Figure 28. A regular end $E$

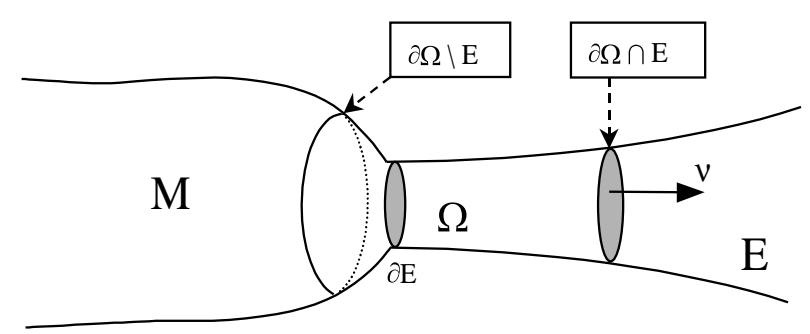

Figure 29. Flux of $u$ on the end $E$

where the constant $C$ does not depend ${ }^{15}$ on $r$.

For example, the end $E$ is regular if it is isometric to an exterior of a ball on a complete Riemannian manifold with a non-negative Ricci curvature (see [124]). $\mathrm{Li}$ and Tam [121, Theorem 3.2] proved that any manifold of non-negative sectional curvature outside a compact set is a manifold with regular ends.

Let $E$ be an end of $M$ and $u$ be a function on $E$. For any precompact open set $\Omega \subset M$ containing $\partial E$ and having a smooth boundary $\partial \Omega$, consider the flux of $u$ through $\partial \Omega \cap E$ (see Figure 29), that is

$$
\operatorname{flux}_{\partial \Omega \cap E} u=\int_{\partial \Omega \cap E} \frac{\partial u}{\partial \nu} d \mu^{\prime} .
$$

If $u$ is harmonic in $E$, then the flux does not depend on $\Omega$, and we can define the flux of $u$ through $E$ by

$$
\underset{E}{\operatorname{flux}} u=\operatorname{flux}_{\partial \Omega \cap E} u
$$

Also, let us set

$$
\lim _{E} u:=\lim _{\substack{x \in E \\ x \rightarrow \infty}} u(x)
$$

provided the limit on the right-hand side exists. It turns out that if $E$ is regular ${ }^{16}$ and $u$ is a non-negative harmonic function on $E$, then the limit (14.9) does exist, finite or infinite (see [74, Proposition 1] or [95, 3.23]). Furthermore, the following theorem holds.

\footnotetext{
${ }^{15}$ If $E_{r}$ is connected, then by the local Harnack inequality and by compactness of $E(r),(14.7)$ is always valid with some $C=C(r)$. The purpose of the regularity hypothesis is to ensure a uniform Harnack constant $C$ as $r \rightarrow \infty$.

${ }^{16}$ Let us note that, for Theorem 14.5 below, the definition of regularity can be slightly relaxed. Namely, instead of being defined by (14.6), the set $U_{r}$ may be any precompact open neighbourhood of $E_{r}$ such that $\operatorname{dist}\left(\partial E, U_{r}\right) \rightarrow \infty$ as $r \rightarrow \infty$. See [95] for further results of this kind.
} 


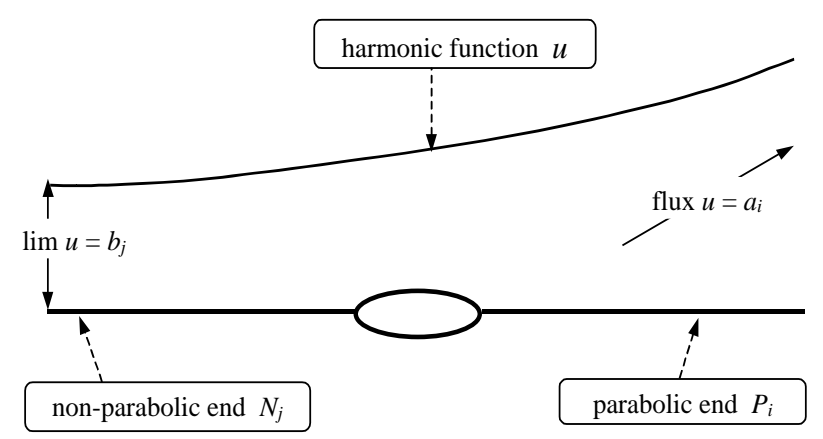

FiguRE 30. Limits and fluxes of a harmonic function $u$

Theorem 14.5. ([74]) Let $M$ be a manifold with regular ends. Assume that it has $s \geq 0$ parabolic ends $P_{i}$ and $l \geq 1$ non-parabolic ends $N_{j}$. Then, for any non-negative harmonic function $u$ on $M$, the numbers $a_{i}, b_{j}$ defined by

$$
a_{i}=\operatorname{flux}_{P_{i}} u \quad \text { and } \quad b_{j}=\lim _{N_{j}} u
$$

( $i=1,2, \ldots, s$ and $j=1,2, \ldots, l)$ exist and are non-negative. Conversely, given a set $\left(a_{1}, a_{2}, \ldots, a_{s}, b_{1}, b_{2}, \ldots, b_{l}\right)$ of $s+l$ non-negative numbers, there is a unique non-negative harmonic function u on $M$ satisfying (14.10) (see Figure 30).

Similarly, any bounded harmonic function $u$ on $M$ is uniquely characterized by a set of real numbers $\left(b_{1}, b_{2}, \ldots, b_{l}\right)$ such that $b_{j}=\lim _{N_{j}} u$.

Remark. If $u$ is a non-negative harmonic function on $M$ and if a flux $a_{i}$ is strictly positive, then one can prove that $\lim _{P_{i}} u=+\infty$; that is, $u$ is unbounded. Therefore, for a bounded $u$, all fluxes $a_{i}$ must vanish, which explains why a bounded harmonic function $u$ is uniquely determined by its limits $b_{j}$ on the non-parabolic ends.

Theorem 14.5 obviously implies that

$$
\operatorname{dim} \mathcal{H}(M)=l
$$

and

$$
\operatorname{dim} \mathcal{H}_{+}(M)=s+l .
$$

As a consequence we see that the Martin boundary of $M$ consists of $s+l$ points.

For a manifold of non-negative sectional curvature outside a compact set, this was proved by Li and Tam [121, Theorem 7.2]. The main part of their proof can be interpreted as the proof of regularity of each end on such a manifold.

See [40], [50], [95], [96], [119], [121], [123], [124], [175] for further results on harmonic functions on manifolds with ends.

14.4. Non-parabolicity of regular ends. We consider here the question how to decide whether a given end $E \subset M$ (so far not necessarily regular) is parabolic or not. Let us denote

$$
B_{r}=\{x \in E: \operatorname{dist}(x, \partial E)<r\} \quad \text { and } \quad V_{E}(r)=\mu\left(B_{r}\right) .
$$


An extension of Theorem 7.3 to manifolds with a boundary (see [71]) says that $E$ is parabolic provided

$$
\int^{\infty} \frac{r d r}{V_{E}(r)}=\infty
$$

It is not known whether (14.11) is necessary for parabolicity of a regular end. As was shown by $\mathrm{Li}$ and Tam [121], if $M$ has a non-negative sectional curvature outside a compact, then parabolicity of its end $E$ is indeed equivalent to (14.11). We discuss below some situations when (14.11) is equivalent to the parabolicity of $E$.

The following statement is essentially an extract from the technique of Li and $\operatorname{Tam}[121]$.

Theorem 14.6. Let $E$ be a regular end. Then $E$ is non-parabolic if and only if

$$
\sum_{k=1}^{\infty} \frac{1}{\operatorname{cap}_{E}\left(B_{2^{k}}, B_{2^{k+1}}\right)}<\infty
$$

Proof. If the sum in (14.12) is divergent, then $E$ is parabolic even without assuming the regularity of $E$. Indeed, capacity satisfies the following universal inequality

$$
\operatorname{cap}_{E}(A, C)^{-1} \geq \operatorname{cap}_{E}(A, B)^{-1}+\operatorname{cap}_{E}(B, C)^{-1},
$$

for any three open precompact sets $A \subset \subset B \subset \subset C$. Therefore, we have, for any $n>1$,

$$
\operatorname{cap}_{E}\left(B_{2^{n}}\right)^{-1} \geq \sum_{k=n}^{\infty} \operatorname{cap}_{E}\left(B_{2^{k}}, B_{2^{k+1}}\right)^{-1}=\infty,
$$

whence $\operatorname{cap}_{E}\left(B_{2^{n}}\right)=0$, and $E$ is parabolic.

Assume now that (14.12) holds and prove that the regular end $E$ is non-parabolic. Denote by $G_{R}$ the Green function in $B_{R}$ with the Neumann condition on $\partial E \cap B_{R}$ and the Dirichlet condition on $E_{R}=\partial B_{R} \cap E$. Fix some reference point $o \in E$ and show that, for any $x \in E, G_{R}(o, x)$ is bounded as $R \rightarrow \infty$, which will imply the non-parabolicity of $E$.

We start with the observation that, for any $x \in B_{r}$ and any $R>r$,

$$
G_{R}(o, x)-G_{r}(o, x) \leq \max _{y \in E_{r}} G_{R}(o, y) .
$$

This follows from the maximum principle for the function $G_{R}(o, \cdot)-G_{r}(o, \cdot)$ which is harmonic in $B(r)$, bounded by the constant $\max _{y \in E_{r}} G_{R}(o, y)$ on $E_{r}$ and satisfies the Neumann boundary condition on $\partial E$. If $R=2 r$ then, by the Harnack inequality (14.7),

$$
\max _{y \in E_{r}} G_{R}(o, y) \leq C \min _{y \in E_{r}} G_{R}(o, y) .
$$

Recall that, by (8.9), we have

$$
\min _{y \in E_{r}} G_{R}(o, y) \leq \operatorname{cap}_{E}\left(B_{r}, B_{R}\right)^{-1} .
$$

Combining (14.16), (14.15) and (14.14), we derive

$$
G_{R}(o, x)-G_{r}(o, x) \leq \frac{C}{\operatorname{cap}_{E}\left(B_{r}, B_{R}\right)} .
$$


Applying (14.17) for $R=2^{k+1}$ and $r=2^{k}$ and iterating for all large $k=m, m+1$, $m+2, \ldots$, we conclude

$$
G(o, x)-G_{2^{m}}(o, x) \leq \sum_{k=m}^{\infty} \frac{C}{\operatorname{cap}_{E}\left(B_{2^{k}}, B_{2^{k+1}}\right)}<\infty
$$

and $G(o, x)<\infty$.

If we know for a regular end $E$ that

$$
\operatorname{cap}_{E}\left(B_{r}, B_{2 r}\right) \geq \operatorname{const} \frac{V_{E}(r)}{r^{2}}
$$

for all large $r$, then (14.12) easily amounts to

$$
\int^{\infty} \frac{r d r}{V_{E}(r)}<\infty
$$

It would be interesting to understand whether (14.18) follows from the hypothesis of regularity of the end. If this is the case, then the assumption (14.12) of Theorem 14.6 can be replaced by (14.19).

Let us consider some examples where (14.18) can be proved.

Example 14.1. For any open set $\Omega \subset E$, denote $\partial_{E} \Omega=\partial \Omega \cap E$. Let us assume that, for any open set $\Omega \subset B_{r}$ with smooth boundary,

$$
\mu^{\prime}\left(\partial_{E} \Omega\right) \geq c \frac{\mu(\Omega)}{r},
$$

for all $r$ large enough and for some $c>0$. Then (14.18) holds, which follows from Theorem 8.1 for the capacitor $\left(B_{r}, B_{2 r}\right)$ with the isoperimetric function $f(v)=\frac{c v}{2 r}$.

Example 14.2. Assume that there exists a non-negative Lipschitz function $\rho(x)$ on E such that

$$
\begin{gathered}
\left.\rho\right|_{\partial E}=0, \\
|\nabla \rho| \leq 1
\end{gathered}
$$

and

$$
\Delta\left(\rho^{2}\right) \geq 2 c,
$$

with a positive constant $c$ ((14.23) is understood in the sense of distributions). For example, such a function was constructed in [121, Propositions 2.1, 2.2] assuming that $E$ is an end of a manifold $M$ with non-negative curvature outside a compact.

Let us verify the isoperimetric inequality (14.20). Integrating (14.23) over $\Omega$, we obtain

$$
c|\Omega| \leq \int_{\Omega} \Delta\left(\rho^{2}\right)=2 \int_{\partial \Omega \cap E} \rho \frac{\partial \rho}{\partial \nu}+\int_{\partial E \cap \Omega} \rho \frac{\partial \rho}{\partial \nu} \leq 2\left(\sup _{B_{r}} \rho\right)\left|\partial_{E} \Omega\right|,
$$

where we have used the Green formula (2.6), (14.22) and (14.21). Since (14.21) and (14.22) imply also $\sup _{B_{r}} \rho \leq r$, we conclude (14.20).

Example 14.3. Assume that the following three hypotheses hold for the end $E$.

(P) Poincaré inequality: for any ball $B(x, 2 r) \subset E$ and for any function $f \in$ $C^{1}(B(x, 2 r))$,

$$
\inf _{\xi \in \mathbb{R}} \int_{B(x, r)}(f(y)-\xi)^{2} d \mu(y) \leq C r^{2} \int_{B(x, 2 r)}|\nabla f|^{2} d \mu .
$$




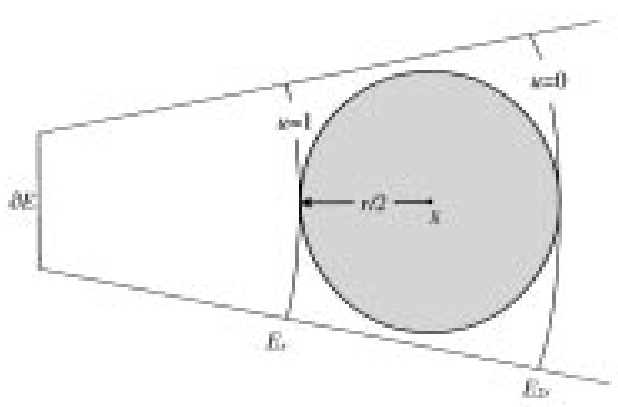

FiguRE 31. Capacitor $\left(B_{r}, B_{2 r}\right)$

(D) Doubling property: for any ball $B(x, 2 r) \subset E$,

$$
V(x, 2 r) \leq C V(x, r) \text {. }
$$

(VC) Volume comparison condition: for any point $x \in E_{r}$,

$$
V_{E}(r) \leq C V(x, r / 2) \text {. }
$$

Let us sketch the proof that $(P),(D)$ and $(V C)$ imply (14.18). The key fact is that $(P)$ and $(D)$ imply the following inequality: for any harmonic function $u$ in $B(x, R) \subset E$,

$$
(\underset{B(x, R / 2)}{\operatorname{osc}} u)^{2} \leq \frac{C R^{2}}{V(x, R)} \int_{B(x, R)}|\nabla u|^{2}
$$

(see [96, Lemma 2.6] for the proof). Let $u$ be the equilibrium potential of the capacitor $\left(B_{r}, B_{2 r}\right)$ and let $x$ be any point on $E_{3 r / 2}$ (see Figure 31 ). Then $u$ is harmonic in $B(x, r / 2)$ and $\underset{B(x, r / 2)}{\text { osc }} u=1$.

By (14.27), we conclude

$$
\operatorname{cap}_{E}\left(B_{r}, B_{2 r}\right)=\int_{B_{2 r} \backslash B_{r}}|\nabla u|^{2} \geq \int_{B(x, r / 2)}|\nabla u|^{2} \geq \operatorname{const} \frac{V(x, r / 2)}{r^{2}} .
$$

Applying $(D)$ and $(V C)$, we have

$$
V(x, r / 2) \geq \operatorname{const} V(x, 3 r / 4) \geq \operatorname{const} V_{E}(3 r / 2) \geq \operatorname{const} V_{E}(r),
$$

whence (14.18) follows.

Holopainen [96, Theorems 2.25 and 4.4] proved that, under the hypotheses $(P)$, $(D)$ and $(V C)$, the parabolicity of $E$ is equivalent to (14.11). His proof does not require regularity of the end. It is plausible that each end satisfying $(P),(D)$ and $(V C)$ can be split into a finite number of regular ends with the comparable volume growth functions (cf. [124, Lemma 1.4]).

Li and Tam [124] proved that $(D)$ and $(P)$ hold on $E$ provided the Ricci curvature on $E$ satisfies

$$
\operatorname{Ric}(x) \geq-\frac{C}{\rho^{2}(x)}, \quad C>0,
$$

where $\rho(x)=\operatorname{dist}(x, \partial E)$. Thus, assuming (14.28) and $(V C)$, one finds that the parabolicity of $E$ is equivalent to (14.11). This is the result of [124, Theorem 1.9]. 


\section{Curvature AND COMPARISON TheOrems}

We prove here some tests for parabolicity and non-explosion in terms of curvature assumptions.

15.1. Mean curvature. Let us fix a point $o$ on a geodesically complete manifold $M$ and consider the polar coordinates $(\rho, \theta)$ centered at $o$ (see Section 3.1). In the domain of the polar coordinates, the Laplace operator is given by (3.4), which we rewrite as

$$
\Delta=\frac{\partial^{2}}{\partial^{2} \rho}+m(\rho, \theta) \frac{\partial}{\partial \rho}+\Delta_{S_{\rho}} .
$$

Here $\Delta_{S_{\rho}}$ is the Laplace operator on the sphere $\partial B(o, \rho)$ and $m(\rho, \theta)$ is a smooth function on $\mathbb{R}_{+} \times \mathbb{S}^{d-1}$, which will be of primary interest for us. In fact, its geometric meaning is the mean curvature of the sphere $\partial B(o, \rho)$ in the radial direction (see [69], [27], [169]).

We will compare the manifold $M$ with a model manifold $M_{\psi}$ introduced in Section 3.2. Let us equip by a hat all notation related to $M_{\psi}$. In particular, we set $\widehat{M}=M_{\psi}$. By (3.6), we have the following expression for a Laplace operator on $\widehat{M}$

$$
\widehat{\Delta}=\frac{\partial^{2}}{\partial^{2} \rho}+\widehat{m}(\rho) \frac{\partial}{\partial \rho}+\widehat{\Delta}_{S_{\rho}},
$$

where

$$
\widehat{m}=(d-1) \frac{\psi^{\prime}}{\psi} .
$$

It is important that $\widehat{m}(\rho)$ does not depend on $\theta$.

Let us consider also the following Schrödinger operators on $M$ and $\widehat{M}$ :

$$
L=\Delta-q(x)
$$

and

$$
\widehat{L}=\widehat{\Delta}-\widehat{q}(\rho)
$$

where the functions $q$ and $\widehat{q}$ are non-negative and continuous.

Theorem 15.1. Let $M$ be a geodesically complete non-compact manifold, and $o \in$ $M$.

(i) Assume that, for all $(\rho, \theta)$ in the domain of the polar coordinates centered at $o$, with $\rho$ being large enough,

$$
m(\rho, \theta) \leq \widehat{m}(\rho) \quad \text { and } \quad q(\rho, \theta) \geq \widehat{q}(\rho) .
$$

If the equation $L u=0$ has a non-zero bounded solution on all of $\widehat{M}$, then so does $\widehat{L} u=0$.

(ii) Let o be a pole. Assume that, for all large enough $\rho$ and all $\theta$,

$$
m(\rho, \theta) \geq \widehat{m}(\rho) \quad \text { and } \quad q(\rho, \theta) \leq \widehat{q}(\rho) .
$$

If the equation $\widehat{L} u=0$ has a non-zero bounded solution on all of $M$, then so does $L u=0$. 


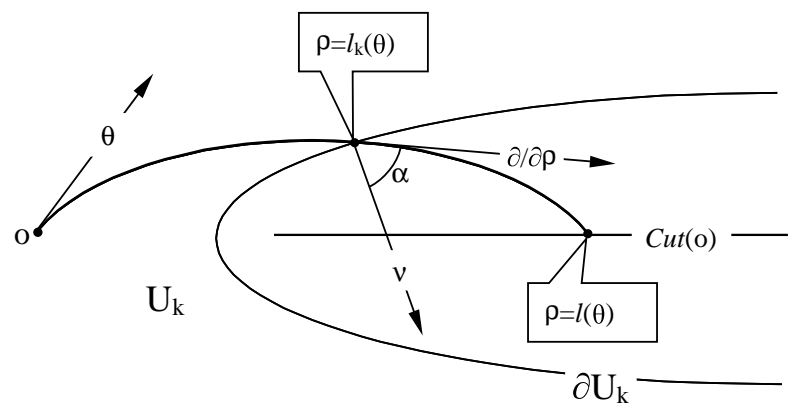

Figure 32. Approximation of the cut locus by a smooth hypersurface

For the case when $o$ is a pole, this theorem was proved in [76]. The technical difficulties which arise due to the cut locus can be handled by the method of Cheeger and Yau [27] developed in the context of comparison of heat kernels (see also [193], [192], [169, Section I.1]).

Proof. (i) Assume that the only bounded solution for the equation $\widehat{L} u=0$ is $u \equiv 0$. Take some $R>0$ and define the function $v(\rho)$ on $[R, \infty)$ to be the solution to the Cauchy problem

$$
v^{\prime \prime}+\widehat{m} v^{\prime}-\widehat{q} v=0, \quad v(R)=0, v^{\prime}(R)=1 .
$$

Then $\widehat{L} v=0$ in $\widehat{\Omega}:=\widehat{M} \backslash \widehat{B}(o, R)$ and, by the maximum principle, the function $v(\rho)$ is monotone increasing. By Theorem 13.6, $v$ must be unbounded, whence $v(\rho) \rightarrow \infty$ as $\rho \rightarrow \infty$ (the converse to Corollary 13.7 for spherically symmetric manifolds).

Let us consider now $v(\rho)$ as a function on $M$. Due to (15.3) and $v^{\prime} \geq 0$, we have in $\Omega \backslash C u t(o)$ (where $\Omega:=M \backslash B(o, R)$ )

$$
L v=v^{\prime \prime}+m v^{\prime}-q v \leq v^{\prime \prime}+\widehat{m} v^{\prime}-\widehat{q} v=\widehat{L} v=0 .
$$

If $C u t(o)$ is empty, then, by Corollary 13.7, this finishes the proof because $L v \leq 0$ in $\Omega$ and $v(x) \rightarrow \infty$ as $x \rightarrow \infty$.

Let $C u t(o)$ be non-empty. We will show that $L v \leq 0$ is still true in $\Omega$ in the sense of distribution (which is enough for Corollary 13.7). More precisely, let us prove that, for any non-negative test function $\phi \in C_{0}^{\infty}(\Omega)$,

$$
\langle L v, \phi\rangle:=-\int_{M}(\nabla v \nabla \phi+q v \phi) d \mu \leq 0 .
$$

For any unit vector $\theta \in T_{o}(M)$, let us define $l(\theta)$ to be the length of the geodesics which starts at $o$ in the direction $\theta$ and ends at $C u t(o)$ (see Figure 32). Since $C u t(o)$ is a closed set, the function $l(\theta)$ is lower semi-continuous. Let $l_{k}(\theta)$ be an increasing sequence of smooth positive functions on the unit sphere which converges to $l(\theta)$ as $k \rightarrow \infty$. Denote by $U_{k}$ the set of points $(\rho, \theta) \in M \backslash C u t(o)$ such that $\rho<l_{k}(\theta)$. The boundary $\partial U_{k}$ is a smooth hypersurface given by the equation $\rho=l_{k}(\theta)$. Clearly, the sequence $\left\{U_{k}\right\}$ is increasing and $\bigcup_{k} U_{k}=M \backslash C u t(o)$.

We have, by the Green formula (2.6),

$$
\int_{U_{k}}(\nabla v \nabla \phi+q v \phi) d \mu=\int_{U_{k}}(-\Delta v \phi+q v \phi) d \mu+\int_{\partial U_{k}} \frac{\partial v}{\partial \nu} \phi
$$


where $\nu$ is the unit outward normal vector field on $\partial \Omega_{k}$. The first term on the right hand side of (15.6) is non-negative because

$$
L v \leq 0 \quad \text { on } \quad U_{k} \cap \operatorname{supp} \phi \subset \Omega \backslash C u t(o) .
$$

We claim that the second term is also non-negative. Indeed, the normal $\nu$ forms an acute angle $\alpha$ with the radial direction $\frac{\partial}{\partial \rho}$, and $\nabla v=v^{\prime}(\rho) \frac{\partial}{\partial \rho}$, whence

$$
\frac{\partial v}{\partial \nu}=\nu \nabla v=\nu\left(v^{\prime}(\rho) \frac{\partial}{\partial \rho}\right)=v^{\prime}(\rho) \cos \alpha \geq 0 .
$$

Thus, (15.6) yields

$$
\int_{U_{k}}(\nabla v \nabla \phi+q v \phi) d \mu \geq 0 .
$$

As $k \rightarrow \infty$, we can replace here $U_{k}$ by $M \backslash C u t(o)$. Finally, $C u t(o)$ has the measure zero, whence we conclude (15.5).

(ii) By Theorem 13.6, there is also a positive bounded solution $u$ to $\widehat{L} u=0$ on $\widehat{M}$. By symmetrizing it, we can assume that $u=u(\rho)$. By the maximum principle, $u(\rho)$ is increasing. Fix some $R>0$, put $v(\rho)=u(\rho)-u(R)$ and observe that $\widehat{L} v \geq 0$.

The function $v$ is positive in the region $\Omega:=\{\rho>R\}$, and, by $v^{\prime} \geq 0$ and (15.4), we have $L v \geq \widehat{L} v \geq 0$ in $\Omega$. Therefore, $v$ is a $q$-subharmonic function, which is bounded, positive in $\Omega$ and vanishing on $\partial \Omega$, whence we see that $\Omega$ is $q$-massive. By Theorem 13.6, there is a non-zero bounded solution to $L u=0$ on $M$.

Combining Theorem 15.1 with the criteria for parabolicity and stochastic completeness in terms of the Liouville properties for the Schrödinger equations (see the beginning of Section 13.2) and with Propositions 3.1, 3.2, we obtain the following statement.

Corollary 15.2. Let $M$ be a geodesically complete non-compact manifold, o $\in M$ and $S(\rho)$ be a positive smooth function on $(0, \infty)$.

(a) If, for all $(\rho, \theta)$ in the domain of the polar coordinates with $\rho$ being large enough,

$$
m(\rho, \theta) \leq \frac{S^{\prime}(\rho)}{S(\rho)} \quad \text { and } \quad \int^{\infty} \frac{d r}{S(r)}=\infty
$$

then $M$ is parabolic (for example, if $m(\rho, \theta) \leq \frac{1}{\rho}$, then the above hypotheses are satisfied with $S(\rho)=\rho)$.

(b) Let $o$ be a pole. If, for all $\rho$ large enough and all $\theta$,

$$
m(\rho, \theta) \geq \frac{S^{\prime}(\rho)}{S(\rho)} \quad \text { and } \quad \int^{\infty} \frac{d r}{S(r)}<\infty,
$$

then $M$ is non-parabolic (for example, if $m(\rho, \theta) \geq \frac{1+\varepsilon}{\rho}$ with $\varepsilon>0$, then the above hypotheses are satisfied with $\left.S(\rho)=\rho^{1+\varepsilon}\right)$.

(c) If, for all $(\rho, \theta)$ in the domain of the polar coordinates with $\rho$ being large enough,

$$
m(\rho, \theta) \leq \frac{S^{\prime}(\rho)}{S(\rho)} \quad \text { and } \quad \int^{\infty} \frac{V(r)}{S(r)} d r=\infty
$$


where

$$
V(r):=\int_{0}^{r} S(\xi) d \xi
$$

then $M$ is stochastically complete (for example, if $m(\rho, \theta) \leq C \rho$, then the above hypotheses are satisfied with $\left.S(\rho)=\exp \left(\frac{1}{2} C \rho^{2}\right)\right)$.

(d) Let o be a pole. If, for all $\rho$ large enough and all $\theta$

$$
m(\rho, \theta) \geq \frac{S^{\prime}(\rho)}{S(\rho)} \quad \text { and } \quad \int^{\infty} \frac{V(r)}{S(r)} d r<\infty,
$$

then $M$ is stochastically incomplete (for example, if $m(\rho, \theta) \geq c \rho^{1+\varepsilon}$ with positive $c, \varepsilon$, then the above hypotheses are satisfied with $\left.S(\rho)=\exp \left(c^{\prime} \rho^{2+\varepsilon}\right)\right)$.

Note that the expression $\frac{S^{\prime}}{S}$ is equal to $\widehat{m}$ for a model manifold $M_{\psi}$ such that $S=\omega_{d} \psi^{d-1}$.

15.2. Sectional and Ricci curvature. Let us turn now to comparison results for the sectional and Ricci curvature. For any $x \in M$ such that $x \notin C u t(o)$ and $x \neq o$, denote by $\operatorname{Ric}_{o}(x)$ the Ricci curvature at $x$ in the direction $\frac{\partial}{\partial \rho}$. Let $\omega$ denote any pair of tangent vectors from $T_{x} M$ having the form $\left(\frac{\partial}{\partial \rho}, X\right)$ where $X$ is a unit vector orthogonal to $\frac{\partial}{\partial \rho}$. Denote by $K_{\omega}(x)$ the sectional curvature at the point $x \in M$ of the 2 -section determined by $\omega$.

Example 15.1. A direct computation [15] yields that, on the model manifold $M_{\psi}$, the curvature $K_{\omega}$ depends only on the polar radius $\rho$ and is given by

$$
K_{\omega}(\rho)=-\frac{\psi^{\prime \prime}}{\psi} .
$$

Respectively, the Ricci curvature $\operatorname{Ric}_{o}$ on $M_{\psi}$ is given by

$$
\operatorname{Ric}_{o}(\rho)=-(d-1) \frac{\psi^{\prime \prime}}{\psi} \text {. }
$$

Theorem 15.3. (Ichihara [99], [100]) Let $\psi(\rho)$ be a smooth positive function on $(0, \infty)$ such that

$$
\psi(0)=0 \quad \text { and } \quad \psi^{\prime}(0)=1 .
$$

Let $M$ be a d-dimensional geodesically complete non-compact manifold, and $o \in M$. Denote

$$
S(r):=\omega_{d} \psi^{d-1}(r)
$$

(a) If, for all $x=(\rho, \theta) \notin C u t(o), x \neq o$,

$$
\operatorname{Ric}_{o}(x) \geq-(d-1) \frac{\psi^{\prime \prime}(\rho)}{\psi(\rho)} \quad \text { and } \quad \int^{\infty} \frac{d r}{S(r)}=\infty,
$$

then $M$ is parabolic.

(b) If $o$ is a pole and, for all $x \neq o$ and all $\omega$,

$$
K_{\omega}(x) \leq-\frac{\psi^{\prime \prime}(\rho)}{\psi(\rho)} \quad \text { and } \quad \int^{\infty} \frac{d r}{S(r)}<\infty,
$$

then $M$ is non-parabolic. 
(c) If, for all $x \notin C u t(o), x \neq o$,

$$
\operatorname{Ric}_{o}(x) \geq-(d-1) \frac{\psi^{\prime \prime}(\rho)}{\psi(\rho)} \quad \text { and } \quad \int^{\infty} \frac{V(r)}{S(r)} d r=\infty
$$

(where $V(r)$ is defined by (15.7)), then $M$ is stochastically complete.

(d) If $o$ is a pole and, for all $x \neq o$ and all $\omega$,

$$
K_{\omega}(x) \leq-\frac{\psi^{\prime \prime}(\rho)}{\psi(\rho)} \quad \text { and } \quad \int^{\infty} \frac{V(r)}{S(r)} d r<\infty,
$$

then $M$ is stochastically incomplete.

Theorem 15.3 can be deduced from Corollary 15.2, applying the Hessian comparison theorem to $M$ and $M_{\psi}$ (see [69, p.19] or [169, Theorem 1.1], or [22, Theorems $3.6,3.8])$. This theorem allows us to compare $m(\rho, \theta)$ and $\widehat{m}(\rho)$ given the comparison of the Ricci curvature or the sectional curvature as above. See [99] and [100] for details.

Let us observe that all parts of Theorem 15.3 require information about the curvature for all $\rho>0$. The following theorem shows that, for stochastic completeness, it suffices to control the curvature only for large $\rho$.

Theorem 15.4. Let $M$ be a geodesically complete manifold.

(a) ( N.Varopoulos [184, Theorem 1], P.Hsu [97]) Assume that $k(r)$ is a positive increasing continuous function on $(0, \infty)$ such that

$$
\int^{\infty} \frac{d r}{k(r)}=\infty
$$

Assume also that, for some point $o \in M$ and for all points $x=(\rho, \theta) \notin C u t(o)$ with $\rho$ large enough, we have

$$
\operatorname{Ric}_{o}(x) \geq-(d-1) k^{2}(\rho) .
$$

Then $M$ is stochastically complete.

(b) (N.Varopoulos [184, Theorem 3], M.Murata [143, Theorem B]) Let $M$ be a manifold with a pole $o$. Assume that $k(r)$ is a non-negative increasing smooth function on $(0, \infty)$ such that

$$
\int^{\infty} \frac{d r}{k(r)}<\infty
$$

and

$$
k^{\prime}(r) \leq C k^{2}(r),
$$

for all $r$ large enough. Assume also that, for all $x=(\rho, \theta) \neq 0$ and all $\omega$,

$$
K_{\omega}(x) \leq-k^{2}(\rho) .
$$

Then $M$ is not stochastically complete.

Remark. Historically, the first results of this kind were obtained by Azencott [6] and Yau [194]. Azencott [6] proved that a Cartan-Hadamard manifold $M$ is stochastically incomplete provided $K_{\omega}(x) \leq-\rho^{2+\varepsilon}, \varepsilon>0$, and $M$ is stochastically complete provided its sectional curvature is uniformly bounded below. Yau [194] proved that an arbitrary geodesically complete manifold is stochastically complete if its Ricci curvature is uniformly bounded below. 
Proof. (a) This part of Theorem 15.4 can be deduced from Theorem 15.3(c). We give another proof, using Theorem 9.1.

If $k(r)$ is bounded, then (15.14) implies $v(o, r) \leq e^{c r}$, and $M$ is stochastically complete by Theorem 9.1. Assume in the sequel that $k(r)$ is unbounded.

By redefining $k(r)$ for small $r$, we may assume that (15.14) holds at any point $x \notin C u t(o), x \neq o$. Since $k(r)$ is monotone increasing for large $r$ and $\lim _{r \rightarrow \infty} k(r)=$ $\infty,(15.14)$ implies

$$
\operatorname{Ric}_{o}(x) \geq-(d-1) k^{2}(R), \quad \forall x \in B(o, R) \backslash C u t(o), \quad x \neq o,
$$

for all $R$ large enough.

By the volume comparison theorem of Bishop (see [22, Theorems 3.8, 3.9]), we obtain, for all $r \leq R$,

$$
V(o, r) \leq \mathrm{V}_{k(R)}(r),
$$

where $\mathrm{V}_{K}(r)$ is the volume of an $r$-ball in the hyperbolic space $\mathbb{H}_{K}^{d}$ of the constant curvature $-K^{2}$. This space is a model manifold $M_{\sigma}$ with $\sigma(r)=K^{-1} \sinh (K r)$, whence we obtain

$$
\mathrm{V}_{K}(r)=\omega_{d} K^{1-d} \int_{0}^{r} \sinh ^{d-1}(K \xi) d \xi<\omega_{d} K^{-d} \exp ((d-1) K r) .
$$

Therefore, letting $r=R$ in (15.18), we obtain, for $R$ large enough,

$$
V(o, R) \leq \text { const } \exp ((d-1) k(R) R)
$$

and

$$
\frac{\log V(o, R)}{R}=O(k(R)), \quad R \rightarrow \infty .
$$

Hence, (15.13) implies

$$
\int^{\infty} \frac{R d R}{\log V(o, R)}=\infty
$$

and the stochastic completeness follows by Theorem 9.1 .

(b) We reduce this part of Theorem 15.4 to Theorem 15.3(d). To that end, we need to construct a function $\psi(r)$ satisfying (15.10) and (15.12). Let $\psi(r)$ solve the following Cauchy problem on $[0, \infty)$

$$
\left\{\begin{array}{l}
\psi(0)=0, \\
\psi^{\prime}(0)=1, \\
\psi^{\prime \prime}=k^{2}(r) \psi .
\end{array}\right.
$$

It is obvious that $\psi(r)>0$ if $r>0$. The hypotheses (15.17) and (15.19) imply that, for all $x \neq o$,

$$
K_{\omega}(x) \leq-\frac{\psi^{\prime \prime}(\rho)}{\psi(\rho)}
$$

Therefore, in order to apply Theorem 15.3(d), we need to verify the second of the conditions (15.12).

Suppose for a moment that we have proved that, for some $\varepsilon>0$ and all $r$ large enough,

$$
\frac{\psi^{\prime}(r)}{\psi(r)} \geq \varepsilon k(r)
$$


This would imply

$$
\frac{S^{\prime}(r)}{S(r)} \geq(d-1) \varepsilon k(r)
$$

where $S(r)=\omega_{d} \psi^{d-1}(r)$. By l'Hospital's rule, we obtain from (15.21), for large $r$,

$$
\frac{S(r)}{V(r)} \geq \operatorname{const} k(r)
$$

where $V(r)=\int_{0}^{r} S(\xi) d \xi$. Finally, (15.22) and (15.15) yield

$$
\int^{\infty} \frac{V(r)}{S(r)} d r<\infty
$$

which, by Theorem 15.3(d), implies the stochastic incompleteness of $M$.

We are left to show that the solution $\psi$ of (15.19) satisfies (15.20). If this is not so, then, for any $\varepsilon_{0}>0$ (to be chosen later), there exist a large enough $r_{0}$ and $\varepsilon \in\left(0, \varepsilon_{0}\right)$ such that

$$
\frac{\psi^{\prime}\left(r_{0}\right)}{\psi\left(r_{0}\right)}=\varepsilon k\left(r_{0}\right)
$$

Denote $v=\frac{\psi^{\prime}}{\psi}$. Then we have

$$
k^{2}=\frac{\psi^{\prime \prime}}{\psi}=\left(\frac{\psi^{\prime}}{\psi}\right)^{\prime}+\left(\frac{\psi^{\prime}}{\psi}\right)^{2}
$$

that is,

$$
v^{\prime}+v^{2}=k^{2}
$$

Compare the function $v$ with $w(r):=\varepsilon k(r)$. We have, by (15.23),

$$
v\left(r_{0}\right)=w\left(r_{0}\right)
$$

and

$$
w^{\prime}+w^{2}=\varepsilon k^{\prime}+\varepsilon^{2} k^{2} .
$$

Choosing $\varepsilon_{0}$ (and thus $\varepsilon$ ) small enough, we obtain, by (15.16),

$$
\varepsilon k^{\prime}+\varepsilon^{2} k^{2} \leq \frac{1}{2} k^{2}
$$

whence

$$
w^{\prime}+w^{2} \leq \frac{1}{2} k^{2} .
$$

Comparing (15.25), (15.24) and (15.26), we conclude $w(r) \leq v(r)$ for all $r$ large enough, which is exactly (15.20). 
15.3. Parabolicity for two dimensional manifolds. Unlike Theorem 15.4, in order to decide whether $M$ is parabolic or not, it is generally not enough to control the curvature $K_{\omega}$ only for large $\rho$. We discuss here to what extent it is still possible.

The curvature $K_{\omega}$ on a model manifold $M_{\psi}$ is computed by (15.8), that is

$$
K_{\omega}(\rho)=-\frac{\psi^{\prime \prime}(\rho)}{\psi(\rho)} .
$$

Suppose that we have another function $\phi$ such that

$$
\frac{\psi^{\prime \prime}(r)}{\psi(r)}=\frac{\phi^{\prime \prime}(r)}{\phi(r)}, \quad \text { for } r>r_{0} .
$$

Then the manifold $M_{\phi}$ will have the same curvature $K_{\omega}(\rho)$ for large $\rho$.

Lemma 15.5. There exists a function $\phi$ satisfying (15.28) and such that one of the integrals

$$
\int^{\infty} \frac{d r}{\psi^{2}(r)}, \quad \int^{\infty} \frac{d r}{\phi^{2}(r)}
$$

is divergent and the other is convergent.

Proof. Given a function $\psi$, it is easy to see that the function

$$
\phi(r)=\psi(r) \int \frac{d r}{\psi^{2}(r)}
$$

satisfies (15.28). Note that we need to define $\psi$ and $\phi$ only for large $r$. The limits in $\int \frac{d r}{\psi^{2}(r)}$ should be chosen so that it is positive and goes to either 0 or to $\infty$ as $r \rightarrow \infty$. Let us show that one of the integrals in (15.29) is divergent and the other is convergent. Define

$$
f=\frac{\phi}{\psi}=\int \frac{d r}{\psi^{2}(r)} \quad \text { and } \quad g=\frac{1}{f} .
$$

Then, by (15.30), $\left|f^{\prime}\right|=\frac{1}{\psi^{2}}$ and $\left|g^{\prime}\right|=\left|\frac{f^{\prime}}{f^{2}}\right|=\frac{1}{\phi^{2}}$. Since both $f^{\prime}$ and $g^{\prime}$ do not change sign, we obtain

$$
\int_{r_{0}}^{\infty} \frac{d r}{\psi^{2}(r)}=\int_{r_{0}}^{\infty}\left|f^{\prime}\right| d r=\left|f(\infty)-f\left(r_{0}\right)\right|
$$

and

$$
\int_{r_{0}}^{\infty} \frac{d r}{\phi^{2}(r)}=\int_{r_{0}}^{\infty}\left|g^{\prime}\right| d r=\left|g(\infty)-g\left(r_{0}\right)\right| .
$$

Clearly, exactly one of the quantities $f(\infty)$ and $g(\infty)=\frac{1}{f(\infty)}$ is infinite (and the other is zero), whence the claim follows.

Suppose that the dimension $d=3$ and $\phi$ is chosen by Lemma 15.5. Then, by Proposition 3.1, one of the manifolds $M_{\psi}, M_{\phi}$ is parabolic whereas the other is not. For example, the functions

$$
\psi(r)=\sqrt{r} \log r \quad \text { and } \quad \phi(r)=\sqrt{r}
$$

satisfy $(15.28), M_{\psi}$ and $M_{\phi}$ have the same curvature profile

$$
K_{\omega}(\rho)=\frac{1}{4 \rho^{2}}, \quad \text { for all large } \rho
$$

whereas $M_{\psi}$ is non-parabolic and $M_{\phi}$ is parabolic. 
Hence, in the dimension $d=3$, the statements (a) and (b) of Theorem 15.3 cannot be true for any function $\psi$ (even in the class of model manifolds) if the curvature assumption is to be assumed only for large $\rho$.

If $d>3$ then one of the integrals

$$
\int^{\infty} \frac{d r}{\psi^{d-1}(r)}, \quad \int^{\infty} \frac{d r}{\phi^{d-1}(r)}
$$

has to be convergent (as follows from Lemma 15.5); that is, one of the manifolds $M_{\psi}, M_{\phi}$ is non-parabolic (note that in the case $d>3$, both integrals in (15.32) can converge, for example, if $\psi(r)=r^{\frac{1-\varepsilon}{2}}$ with $\left.|\varepsilon|<\frac{d-3}{d-1}\right)$.

Hence, if $d>3$ then one cannot claim the parabolicity of a model manifold given the curvature $K_{\omega}(\rho)$ for large $\rho$. Neither can one claim the non-parabolicity of a model manifold given an upper bound for $K_{\omega}(\rho)$ (as in Theorem 15.3(b)) only for large $\rho$. Indeed, let us set $\psi(r)=r^{\alpha}$ and $\chi(r)=r^{\beta}$, where $0<\beta<\frac{1}{d-1}<\alpha<\frac{1}{2}$. Then, for the model manifold $M_{\chi}$,

$$
K_{\omega}(\rho)=-\frac{\chi^{\prime \prime}}{\chi}=\frac{\beta(1-\beta)}{\rho^{2}}<\frac{\alpha(1-\alpha)}{\rho^{2}}=-\frac{\psi^{\prime \prime}}{\psi} .
$$

Despite $\int^{\infty} \frac{d r}{\psi^{d-1}(r)}<\infty$, the manifold $M_{\chi}$ is parabolic, due to $\beta<\frac{1}{d-1}$.

This discussion shows that in order to extend the statements (a) and (b) of Theorem 15.3 to situations where the curvature is controlled only for large $\rho$, one has to involve some non-curvature hypotheses. To the best of our knowledge, such results are unknown yet, except for the case $d=2$. The next theorem shows that, in the dimension $d=2$, in order to deduce the parabolicity of $M$, it suffices to control the curvature only for large $\rho$, whereas for the non-parabolicity, a certain additional assumption is required.

We suppress the subscript $\omega$ in $K_{\omega}(x)$ because in the case $d=2$, there is only one $\omega$, and $K(x)$ is the Gauss curvature at $x$.

Theorem 15.6. ([84]) Let $M$ be a two-dimensional geodesically complete manifold and $o \in M$. Let $\psi(\rho)$ be a smooth positive increasing function on $(1, \infty)$.

(a) Assume that, for all $x \in M$ with $\rho:=\operatorname{dist}(x, o)$ being large enough,

$$
K(x) \geq-\frac{\psi^{\prime \prime}(\rho)}{\psi(\rho)}
$$

and

$$
\int^{\infty} \frac{d r}{\psi(r)}=\infty
$$

Then $M$ is parabolic.

(b) Let o be a pole. Assume that, for all $\rho$ large enough and all $\theta \in \mathbb{S}^{1}$,

$$
K(\rho, \theta) \leq-\frac{\psi^{\prime \prime}(\rho)}{\psi(\rho)}
$$

and

$$
\int^{\infty} \frac{d r}{\psi(r)}<\infty
$$

Assume in addition that

$$
\limsup _{r \rightarrow \infty} L(r)>0,
$$


where $L(r):=\mu^{\prime}(\partial B(o, r))$. Then $M$ is non-parabolic.

Remark. The hypothesis (15.37) allows us to exclude the "dual" manifold $M_{\phi}$ where $\phi$ is defined by (15.30). For example, if $\psi(r)=r^{\alpha}$, where $\alpha>1$, then $\phi(r)=c r^{1-\alpha}$ and $L(r)=2 \pi \phi(r) \rightarrow 0$ as $r \rightarrow \infty$, so that (15.37) fails to hold for $M=M_{\phi}$. Clearly, $M_{\phi}$ is parabolic, despite both (15.35) and (15.36) being true for $M_{\phi}$.

Example 15.2. Let us set $\psi(r)=r \log r$. Then the parabolicity test (15.33) becomes

$$
K(\rho, \theta) \geq-\frac{1}{\rho^{2} \log \rho} .
$$

Let us set $\psi(r)=r \log ^{1+\varepsilon} r, \varepsilon>0$, and assume that $M$ is a manifold with a pole, satisfying (15.37). Then the non-parabolicity test (15.35) becomes

$$
K(\rho, \theta) \leq-\frac{1+\varepsilon}{\rho^{2} \log \rho} .
$$

These two tests were first proved by Milnor [139] for model manifolds and then by Doyle [52] for manifolds with a pole. The proof of Theorem 15.6 below is also inspired by these two works.

Proof of Theorem 15.6. (a) In the domain of the polar coordinates, the Riemannian metric of $M$ has the form (3.1), which in the case $d=2$ amounts to

$$
d s^{2}=d \rho^{2}+\sigma^{2}(\rho, \theta) d \theta^{2},
$$

where $\sigma(\rho, \theta)$ is a smooth positive function on $\mathbb{R}_{+} \times \mathbb{S}^{1}$. A straightforward computation of the Gauss curvature yields

$$
K(\rho, \theta)=-\frac{\sigma^{\prime \prime}(\rho, \theta)}{\sigma(\rho, \theta)},
$$

where $\sigma^{\prime \prime}$ means $\partial^{2} \sigma / \partial \rho^{2}$. The hypothesis (15.33) implies

$$
\frac{\sigma^{\prime \prime}(\rho, \theta)}{\sigma(\rho, \theta)} \leq \frac{\psi^{\prime \prime}(\rho)}{\psi(\rho)}
$$

in the domain of the polar coordinates and for $\rho$ large enough.

Let us denote

$$
L(r):=\int_{\mathbb{S}^{1}} \sigma(r, \theta) d \theta ;
$$

that is $L(r)$ is the length of the geodesic circle $\partial B(o, r)$ outside the cut locus $C u t(o)$. Since the cut locus has measure zero, we have

$$
L(r)=\frac{d}{d r} V(o, r) .
$$

The parabolicity of $M$ will follow by Theorem 7.5 if we prove that

$$
\int^{\infty} \frac{d r}{L(r)}=\infty .
$$

Integrating (15.39) in $\theta$, pre-multiplied by $\sigma \psi$, we obtain

$$
L^{\prime \prime} \psi-L \psi^{\prime \prime} \leq 0,
$$

whence

$$
\left(L^{\prime} \psi-L \psi^{\prime}\right)^{\prime} \leq 0,
$$


and the function $L^{\prime}(r) \psi(r)-L(r) \psi^{\prime}(r)$ is decreasing, for large $r$. Therefore, it is bounded by a constant $C$, which yields

$$
\left(\frac{L}{\psi}\right)^{\prime}=\frac{L^{\prime} \psi-L \psi^{\prime}}{\psi^{2}} \leq \frac{C}{\psi^{2}}
$$

Integrating (15.41) from $r_{0}$ (being a large enough number) to $r>r_{0}$, we obtain

$$
\frac{L}{\psi}(r) \leq \frac{L}{\psi}\left(r_{0}\right)+C \int_{r_{0}}^{r} \frac{d \xi}{\psi^{2}(\xi)}
$$

Assume first that $\psi$ satisfies

$$
\int^{\infty} \frac{d r}{\psi^{2}(r)}<\infty
$$

Then the right-hand side of (15.42) is bounded by a constant, and we obtain $L(r) \leq$ const $\psi(r)$. Therefore, (15.40) follows from the hypothesis (15.34).

If $\psi$ does not satisfy (15.43), that is, if

$$
\int^{\infty} \frac{d r}{\psi^{2}(r)}=\infty
$$

then we define the function $\phi$ by

$$
\phi(r)=\psi(r) \int_{r_{0}}^{r} \frac{d \xi}{\psi^{2}(\xi)}
$$

and rewrite (15.42) as

$$
L(r) \leq \frac{L\left(r_{0}\right)}{\psi\left(r_{0}\right)} \psi(r)+C \phi(r)
$$

Since (15.44) and (15.45) imply $\psi(r)=o(\phi(r))$ as $r \rightarrow \infty$, (15.46) yields, for $r$ large enough, $L(r) \leq$ const $\phi(r)$.

We are left to verify that

$$
\int^{\infty} \frac{d r}{\phi(r)}=\infty
$$

Since $\psi$ is increasing, we may assume that $c:=\inf _{\left(r_{0}, \infty\right)} \psi>0$, whence $\psi(r) \leq$ $c^{-1} \psi^{2}(r)$. By setting $f:=\frac{\phi}{\psi}=\int_{r_{0}}^{r} \frac{d \xi}{\psi^{2}(\xi)}$, we obtain

$$
\begin{aligned}
\int^{\infty} \frac{d r}{\phi(r)} & =\int^{\infty} \frac{d r}{f(r) \psi(r)} \geq \int^{\infty} \frac{c d r}{f(r) \psi^{2}(r)} \\
& =c \int^{\infty} \frac{f^{\prime}(r) d r}{f(r)}=c \log f(\infty)+\text { const }=\infty
\end{aligned}
$$

(b) In the previous notation, we have now

$$
\frac{\sigma^{\prime \prime}(\rho, \theta)}{\sigma(\rho, \theta)} \geq \frac{\psi^{\prime \prime}(\rho)}{\psi(\rho)}
$$

for all $\rho$ large enough. By Theorem 12.1, the non-parabolicity of a manifold with a pole follows from

$$
\int_{\mathbb{S}^{1}} \frac{d \theta}{\int_{1}^{\infty} \sigma^{-1}(r, \theta) d r}>0
$$


which is equivalent to

$$
\text { meas }\left\{\theta \in \mathbb{S}^{1}: \int_{1}^{\infty} \frac{d r}{\sigma(r, \theta)}<\infty\right\}>0 .
$$

As in the previous proof, we see that the function $\sigma^{\prime}(r, \theta) \psi(r)-\psi^{\prime}(r) \sigma(r, \theta)$ is increasing in $r$ and, therefore, is bounded below by a constant $-C$, uniformly for all $\theta \in \mathbb{S}^{1}$ and $r \geq R$, where $R$ is a large enough number. This implies

$$
\left(\frac{\sigma}{\psi}\right)^{\prime}=\frac{\sigma^{\prime} \psi-\psi \sigma^{\prime}}{\psi^{2}} \geq-\frac{C}{\psi^{2}} .
$$

The hypothesis (15.36) and the monotonicity of $\psi$ imply (15.43). Therefore, we obtain from (15.50), for all $r \geq r_{0} \geq R$,

$$
\frac{\sigma(r, \theta)}{\psi(r)} \geq \frac{\sigma\left(r_{0}, \theta\right)}{\psi\left(r_{0}\right)}-C \int_{r_{0}}^{\infty} \frac{d \xi}{\psi^{2}(\xi)} .
$$

Defining the function $\phi$ by

$$
\phi(r)=\psi(r) \int_{r}^{\infty} \frac{d \xi}{\psi^{2}(\xi)},
$$

we rewrite (15.51) as

$$
\frac{\sigma(r, \theta)}{\psi(r)} \psi\left(r_{0}\right) \geq \sigma\left(r_{0}, \theta\right)-C \phi\left(r_{0}\right) .
$$

Let us split the set of all $\theta$ into two parts:

$$
\Theta_{0}=\left\{\theta \in \mathbb{S}^{1}: \sigma(r, \theta)<2 C \phi(r), \quad \text { for all } r \geq R\right\}
$$

and $\Theta_{1}=\mathbb{S}^{1} \backslash \Theta_{0}$.

If $\theta \in \Theta_{1}$ then, for some $r_{0} \geq R$, we have

$$
\sigma\left(r_{0}, \theta\right) \geq 2 C \phi\left(r_{0}\right) .
$$

By (15.53), we obtain, for any $r \geq r_{0}$,

$$
\frac{\sigma(r, \theta)}{\psi(r)} \psi\left(r_{0}\right) \geq C \phi\left(r_{0}\right),
$$

whence $\sigma(r, \theta) \geq$ const $\psi(r)$ and, by (15.36),

$$
\int_{1}^{\infty} \frac{d r}{\sigma(r, \theta)}<\infty
$$

We are left to show that meas $\left(\Theta_{1}\right)>0$, which will imply (15.49).

If $\theta \in \Theta_{0}$ then, by (15.54), $\sigma(r, \theta) \leq 2 C \phi(r)$, for $r$ large enough, whence

$$
\int_{\Theta_{0}} \sigma(r, \theta) d \theta \leq \operatorname{const} \phi(r) .
$$

Since $\psi$ is increasing, we have, by (15.52) and (15.36),

$$
\phi(r) \leq \int_{r}^{\infty} \frac{d \xi}{\psi(\xi)} \longrightarrow 0, \quad r \rightarrow \infty .
$$

Thus, (15.56) and (15.37) imply

$$
\limsup _{r \rightarrow \infty} \int_{\Theta_{1}} \sigma(r, \theta) d \theta>0,
$$

whence $\Theta_{1}$ has positive measure. 
Remark. As is clear from the proof, the hypothesis (15.37) can be relaxed as follows:

$$
\limsup _{r \rightarrow \infty} \frac{L(r)}{\phi(r)}=\infty
$$

where $\phi$ is defined by (15.52). For example, if $\psi(r)=r \log ^{\alpha} r, \alpha>1$, then $\phi(r) \asymp$ $\log ^{-\alpha} r$. Therefore, (15.37) can be replaced in this case by

$$
\limsup _{r \rightarrow \infty} L(r) \log ^{\alpha} r=\infty
$$

\section{HEAT KERNEL'S LOWER BOUNDS AND RECURRENCE OF $\alpha$-PROCESS}

Given a lower bound of the heat kernel on $M$, one may be able to prove that $M$ is parabolic, using Theorem 5.1(5). The latter says that $M$ is parabolic provided

$$
\int^{\infty} p(t, x, x) d t=\infty
$$

In general, obtaining pointwise lower bounds of the heat kernel $p(t, x, y)$ may be difficult and requires restrictive geometric assumptions (see [126], [78], [164], [165]). However, the parabolicity test (16.1) requires only an on-diagonal lower bound, which is much easier. The following theorem provides such an estimate assuming only a knowledge of the volume growth.

Theorem 16.1. (Coulhon and Grigor'yan [36, Theorem 6.1], [35]) Let $M$ be geodesically complete. Assume that, for some point $x \in M$ and all $r \geq r_{0}>0$,

$$
V(x, r) \leq C r^{N}
$$

for some (large) positive $C, N$. Then, for all $t \geq t_{0}=t_{0}\left(r_{0}\right)>0$,

$$
p(t, x, x) \geq \frac{0.5}{V(x, \sqrt{a t \log t})},
$$

with some positive constant a which depends on $C, N$ as well as on certain intrinsic properties of the ball $B\left(x, r_{0}\right)$.

As an immediate consequence, we see that the hypothesis

$$
V(x, r) \leq C r^{2}
$$

implies the parabolicity of $M$. Indeed, (16.3) and (16.2) imply, for large $t$,

$$
p(t, x, x) \geq \frac{\mathrm{const}}{t \log t},
$$

whence (16.1) follows.

However, this method does not give the sharp integral condition (7.12) because of the excessive $\log t$ in the estimate (16.2). As was shown in [36, Section 9], this $\log t$ cannot be eliminated.

Given a pointwise on-diagonal lower bound of the heat kernel, we can get for free the recurrence of some stochastic processes other than the Brownian motion. It is known [135, Theorem 3.2] that for any $\alpha \in(0,2)$, the operator $(-\Delta)^{\alpha / 2}$ is a generator of a Hunt process on $M$. Let us call it the $\alpha$-process. It is a natural generalization of the $\alpha$-stable process in $\mathbb{R}^{d}$. As follows from a general semigroup 
theory of subordinated processes ${ }^{17}$ (see [195, IX.11] and [135, p.234]) the Green function $G^{(\alpha)}(x, y)$ of $(-\Delta)^{\alpha / 2}$ is given by

$$
G^{(\alpha)}(x, y)=\int_{0}^{\infty} t^{\alpha / 2-1} p(t, x, y) d t
$$

and the recurrence of the $\alpha$-process is equivalent to $G^{(\alpha)} \equiv \infty$. The latter amounts easily to

$$
\int^{\infty} t^{\alpha / 2-1} p(t, x, x) d t=\infty
$$

(cf. the proof of Theorem 5.1, part $(4) \Longleftrightarrow(5)$ ).

For example, if

$$
V(x, r) \leq C r^{\alpha},
$$

then, by Theorem 16.1,

$$
p(t, x, x) \geq \frac{\text { const }}{t^{\alpha / 2} \log ^{\alpha / 2} t},
$$

and we see that (16.4) is satisfied. Thus, we have

Theorem 16.2. Let $M$ be a geodesically complete manifold, and assume that (16.5) holds for some $x$ and all large $r$. Then the $\alpha$-process is recurrent.

A slightly weaker result was proved by I.McGillivray [135].

In the same way, we derive the following statement from the estimates (11.4) and (11.5) of the heat kernel.

Theorem 16.3. Let $M$ be geodesically complete and assume that the relative FaberKrahn inequality (11.2) holds on $M$. Then the recurrence of the $\alpha$-process is equivalent to

$$
\int^{\infty} \frac{d t}{V\left(x, t^{1 / \alpha}\right)}=\infty
$$

Similarly, one can produce a transience test for the $\alpha$-process by using the upper bound of the heat kernel (10.16).

Theorem 16.4. Let $M$ satisfy a uniform Faber-Krahn inequality (10.5) and assume

$$
\int^{\infty}\left[\int_{1}^{v} \frac{d \xi}{\xi \Lambda(\xi)}\right]^{\alpha / 2-1} \frac{d v}{v^{2} \Lambda(v)}<\infty .
$$

Then the $\alpha$-process is transient.

In particular, if $\Lambda(v) \sim v^{-\beta}$, for large $v$, then (16.6) holds if and only if $\alpha \beta<2$. A nice application of this theorem occurs on a manifold of bounded geometry which is, by definition, a manifold with Ricci curvature bounded below and with a positive injectivity radius. A complete manifold of bounded geometry always admits a Faber-Krahn inequality with the function

$$
\Lambda(v)=\operatorname{const} v^{-2},
$$

\footnotetext{
${ }^{17}$ The author is obliged to Ivor McGillivray for explaining the particulars of subordinated processes.
} 
for large enough $v$; see [82, Theorem 2.1]. ${ }^{18}$ Therefore, if $\alpha<1$ then the $\alpha$-process is transient on any complete manifold of bounded geometry.

\section{Escape Rate AS A MEASURe of TRANSIENCE}

The transience of the process $X_{t}$ means that, with $\mathbb{P}_{x}$-probability 1 , it leaves any ball $B(x, r)$ forever after some (random) $t$. One may wonder if the radius $r$ can be time-dependent. In other words, is there an increasing function $r(t)$ such that $X_{t} \notin B(x, r(t))$, for all $t$ large enough $\mathbb{P}_{x}$-a.s.? We will call such a function $a$ lower radius for $X_{t}$. A sphere $\partial B(x, r(t))$ can then be regarded as a rear front of the diffusion process.

There is a natural counterpart to a lower radius - an upper radius. An increasing function $R(t)$ is called an upper radius if, with $\mathbb{P}_{x}$-probability 1 , we have $X_{t} \in$ $B(x, R(t))$, for all $t$ large enough. The sphere $\partial B(x, R(t))$ can be regarded as a forefront of the Brownian motion (see Figure 33).

A sharp estimate of a lower radius in $\mathbb{R}^{d}$ was obtained by Dvoretzky and Erdös [54]. Namely, if $r(r) / \sqrt{t}$ is a decreasing function, then $r(t)$ is a lower radius for the Brownian motion in $\mathbb{R}^{d}, d>2$, if and only if

$$
\int^{\infty}\left(\frac{r(t)}{\sqrt{t}}\right)^{d-2} \frac{d t}{t}<\infty
$$

In particular, the function

$$
r(t)=\frac{C \sqrt{t}}{\log ^{\frac{1+\varepsilon}{d-2}} t}, \quad C>0
$$

is a lower radius if $\varepsilon>0$ and is not if $\varepsilon \leq 0$. Note that the restriction $d>2$ is essential; otherwise the process is recurrent, and there is no increasing lower radius.

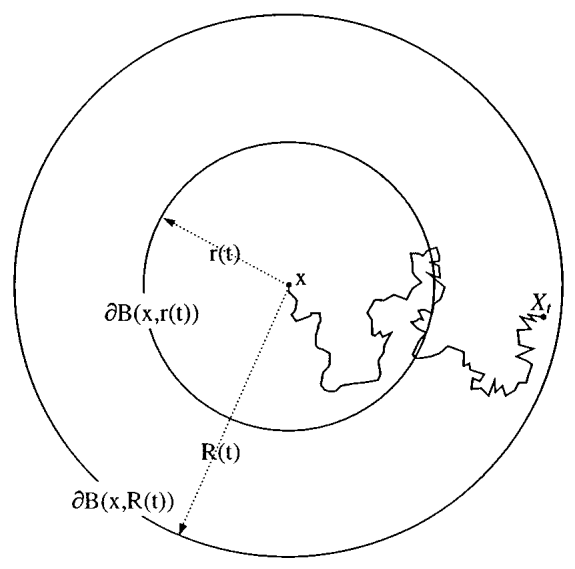

Figure 33. A lower radius $r(t)$ and an upper radius $R(t)$

\footnotetext{
${ }^{18}$ In fact, the hypothesis of a positive injectivity radius can be relaxed as to the assumption that, for some positive $\rho, v_{0}$, all balls of radius $\rho$ have the volume at least $v_{0}>0$. Note that the function (16.7) is the same as in $\mathbb{R}^{1}$ and as in a cylinder $\mathbb{R}^{1} \times K$ where $K$ is compact. It reflects the fact that a manifold of bounded geometry has at least dimension 1 at infinity. See [185], [24], [33].
} 
The celebrated Khinchin's theorem - the law of the iterated logarithm - says that the function

$$
R(t)=\sqrt{(2+\varepsilon) t \log \log t}
$$

is an upper radius in $\mathbb{R}^{d}$ if $\varepsilon>0$ and is not if $\varepsilon \leq 0$ (see [103, Section 4.12] and [14] for further results in this direction).

We briefly outline similar results for Riemannian manifolds.

Theorem 17.1. (the law of the single logarithm - [86], [83]) Let $M$ be a geodesically complete manifold of at most polynomial volume growth; i.e., for some $x \in M$ and all $r$ large enough,

$$
V(x, r) \leq \operatorname{const} r^{N}
$$

with some $N>0$. Then the function

$$
R(t)=\sqrt{2 N t \log t}
$$

is an upper radius for $X_{t}$.

Note that there are counterexamples which show that the function (17.4) cannot in general be replaced by $\sqrt{C t \log \log t}$ - see [8], [87]. However, if the relative FaberKrahn inequality (11.2) holds, then one does have the upper radius (17.3) - see [86, Theorem 1.3].

For a lower radius, there is the following test.

Theorem 17.2. ([83, Theorem 5.1]) Let $M$ be geodesically complete and let us assume that the relative Faber-Krahn inequality (11.2) holds on M. Assume also that $M$ is non-parabolic and denote, for some $x \in M$,

$$
\gamma(r):=\left(\int_{r}^{\infty} \frac{s d s}{V(x, s)}\right)^{-1}
$$

Let $r(t)$ be an increasing positive function on $(0, \infty)$ such that

$$
\int^{\infty} \frac{\gamma(r(t))}{V(x, \sqrt{t})} d t<\infty
$$

Then $r(t)$ is a lower radius for the process $X_{t}$ started at $x$.

The hypothesis of non-parabolicity ensures that the integral in (17.5) is convergent. Moreover, given the relative Faber-Krahn inequality, the non-parabolicity of $M$ is equivalent to the convergence of the integral in (17.5) as is stated by Theorem 11.1 .

Let us recall that the relative Faber-Krahn inequality holds on manifolds of non-negative Ricci curvature. Thus, Theorem 17.2 applies on such manifolds.

Example 17.1. Let $V(x, r) \sim r^{\nu}$ for large $r$ and some $\nu>2$. We obtain from (17.5) $\gamma(t) \sim t^{\nu-2}$, and (17.6) amounts to

$$
\int^{\infty} \frac{r^{\nu-2}(t) d t}{t^{\nu / 2}}<\infty
$$

which coincides with the Dvoretzky-Erdös condition (17.1) for $d=\nu$. 
Example 17.2. Let $V(x, r) \sim r^{2} \log ^{\nu} r$ for large $r$ with some $\nu>1$. We find $\gamma(t) \sim$ $\log ^{\nu-1} t$ for large $t$, and (17.6) acquires the form

$$
\int^{\infty} \frac{\log ^{\nu-1} r(t)}{t \log ^{\nu} t} d t<\infty
$$

For example, we can put $r(t)=t^{(\log \log t)^{-\alpha}}$, for large $t$, with any $\alpha>\frac{1}{\nu-1}$.

See [4], [5, p.60], [83], [86], [93] for other situations when the escape rate can be estimated.

\section{Problem SECTion}

We suggest here some open problems related to the topics of the paper. Their difficulty varies from very hard to apparently accessible for graduate students specializing in the area.

Manifold $M$ is always assumed geodesically complete and non-compact.

Parabolicity

1. Let $M$ be a manifold with a pole and $(\rho, \theta)$ be the polar coordinates on $M$. Theorem 12.1 says that $M$ is non-parabolic provided

$$
\text { meas }\left(\theta \in \mathbb{S}^{d-1}: \int^{\infty} \frac{d \rho}{D(\rho, \theta)}<\infty\right)>0
$$

where $D(\rho, \theta)$ is the angular density of the boundary area function (see (12.2)). Prove (or disprove) the converse: if, for almost all $\theta \in \mathbb{S}^{d-1}$,

$$
\int^{\infty} \frac{d \rho}{D(\rho, \theta)}=\infty
$$

then $M$ is parabolic.

If this is not true, then find another condition which would be both necessary and sufficient for the parabolicity of $M$. The question is open even for the twodimensional $M$.

2. Let a smooth hypersurface $\Gamma$ divide a manifold $M$ into two open subsets $N_{1}, N_{2}$. Suppose that each subset $N_{1}, N_{2}$ is parabolic (see Section 14.1 for the definition of parabolic subsets). Is it true that $M$ is parabolic?

If $\Gamma$ is compact, then the affirmative answer is given by Proposition 14.1(e). If $\Gamma$ is not compact, then some additional hypotheses about the structure of $\Gamma$ may be required.

The same question for $M$ being partitioned into a finite number of open subsets $N_{1}, N_{2}, \ldots, N_{n}$ : when is it true that $M$ is parabolic provided all sets $N_{i}$ are parabolic? This is related to Question 1. Indeed, let the sphere $\mathbb{S}^{d-1}$ be partitioned into cells $\omega_{1}, \ldots, \omega_{n}$. This induces partitioning of $M$ into the cones $N^{\omega_{i}}=\left\{(\rho, \theta): \rho>0, \theta \in \omega_{i}\right\}$. Assume that, in any cone $N^{\omega_{i}}$, the boundary area density $D(\rho, \theta)$ is given by $D(\rho, \theta)=D_{i}(\rho)$, neglecting the smoothness of $D$ on the boundaries $\partial N^{\omega_{i}}$. Then the parabolicity of $N^{\omega_{i}}$ is equivalent to

$$
\int^{\infty} \frac{d \rho}{D_{i}(\rho)}=\infty
$$

(cf. Lemma 12.2). Suppose that (18.1) holds for all $i=1,2, \ldots, n$. Is it true that $M$ is parabolic? 
If we knew that

$$
\int^{\infty} \frac{d \rho}{\sum_{i} D_{i}(\rho)\left|\omega_{i}\right|}=\infty
$$

then $M$ would be parabolic by Theorem 7.5. However, (18.1) does not necessarily imply (18.2) (cf. Example 7.3).

3. How to extend Theorem 12.1 to arbitrary geodesically complete manifolds (without a pole)? This amounts to obtaining a lower bound of capacity generalizing (12.6). Let $v(x)$ be a smooth exhaustion function on $M$ and let $B_{r}:=$ $\{x \in M: v(x)<r\}$. A heuristic analogue of (12.6) would be the estimate

$$
\operatorname{cap}\left(B_{r}, B_{R}\right) \geq \int\left(\int_{r}^{R} \frac{(d \rho)^{2}}{|\nabla v|^{2} d \mu}\right)^{-1},
$$

which, however, does not make sense in this form. It is obtained similarly to the proof of Theorem 12.1, by splitting $B_{R} \backslash B_{r}$ into thin tubes connecting $\partial B_{r}$ and $\partial B_{R}$ and estimating the capacity inside each tube by

$$
\operatorname{cap}\left(B_{r}^{\omega}, B_{R}^{\omega}\right) \cong\left(\int_{r}^{R} \frac{d \rho}{\operatorname{flux}_{\partial B_{\rho}^{\omega} v}}\right)^{-1}
$$

(cf. (7.6) and Lemma 12.2). For a small piece of surface $\partial B_{\rho}^{\omega}$, we have

$$
\operatorname{flux}_{\partial B_{\rho}^{\omega}} v \cong|\nabla v| d \mu^{\prime} \cong|\nabla v|^{2} \frac{d \mu}{d \rho},
$$

whence

$$
\operatorname{cap}\left(B_{r}^{\omega}, B_{R}^{\omega}\right) \cong\left(\int_{r}^{R} \frac{(d \rho)^{2}}{|\nabla v|^{2} d \mu}\right)^{-1},
$$

and (18.3) follows by summing up the above estimate over all tubes. The question is whether it is possible to make all this rigorous.

4. Let $M$ be a geodesically complete manifold with a pole $o$. Denote $S(r)=$ $\mu^{\prime}(\partial B(o, r))$ and assume that

$$
\int^{\infty} \frac{d r}{S(r)}<\infty
$$

Suppose also that

$$
K_{\omega}(x) \leq-\frac{\psi^{\prime \prime}(\rho)}{\psi(\rho)}, \quad \text { for all large enough } \rho:=\operatorname{dist}(x, o),
$$

where $K_{\omega}(x)$ is the sectional curvature as in Section 15.2 and $\psi$ is a function as in Theorem 15.3 and such that

$$
\int^{\infty} \frac{d r}{\psi^{d-1}(r)}<\infty
$$

Is it true that $M$ is non-parabolic?

Example 7.3 shows that (18.4) alone is not sufficient for the non-parabolicity of $M$. As was explained in Section 15.3, (18.5) and (18.6) do not imply the nonparabolicity either (unless (18.5) holds for all $\rho>0$ - see Theorem 15.3(b)). If $d=2$ then all three conditions (18.4), (18.5) and (18.6) do imply that $M$ is non-parabolic, by Theorem 15.6(b). The question is whether the same is true for $d>2$. 
Consider the particular case $d=3$ and $\psi=r^{\alpha}$ with $\alpha>1 / 2$, which ensures (18.6). Then the question becomes as follows: is it true that (18.4) and

$$
K_{\omega}(x) \leq \frac{1-\varepsilon}{4 \rho^{2}}, \quad \text { for all large enough } \rho,
$$

where $\varepsilon>0$, imply that $M$ is non-parabolic?

5. (Milnor [139]) Let $M$ be a graph of a smooth function $z=f(x, y)$ defined for all $(x, y) \in \mathbb{R}^{2}$. Find a criterion for parabolicity of $M$ in terms of the function $f$. Examples of non-parabolic surfaces of this kind can be found in [156].

6. Consider a domain of revolution in $\mathbb{R}^{d}$ :

$$
\mathcal{D}_{f}:=\left\{x \in \mathbb{R}^{d}: 0 \leq x_{1}<\infty \quad \text { and } \quad\left|x^{\prime}\right| \leq f\left(x_{1}\right)\right\},
$$

where $x^{\prime}=\left(x_{2}, \ldots, x_{d}\right)$ and $f$ is a smooth function. The set $\mathcal{D}_{f}$ can be considered as a manifold with boundary (make it smooth near the origin). Find a criterion for parabolicity of $\mathcal{D}_{f}$ in terms of the function $f$.

If $f$ is "slowly" changing, then one expects that the parabolicity of $\mathcal{D}_{f}$ is equivalent to

$$
\int^{\infty} \frac{d x}{f^{d-1}(x)}=\infty
$$

that is analogous to Proposition 3.1. However, if $f$ oscillates then it is not clear whether (18.8) is a good guess.

Explosion

7. Does there exist an explosion criterion which would be similar to the capacity criterion for transience given by Theorem 5.1(6)? T.Lyons [131] constructed an example of a manifold where the explosion is not stable under a quasi isometry. Therefore, the corresponding "capacity" should not be a quasi-isometric invariant either.

8. Theorem 5.7(b) says that $M$ is non-parabolic if and only if there exists a smooth vector field $v$ on $M$ such that $|v| \in L^{2}(M, \mu), \operatorname{div} v \in L^{1}(M, \mu)$ and

$$
\int_{M} \operatorname{div} v d \mu \neq 0 \text {. }
$$

Find a criterion for explosion similar to the above criterion for transience. A statement should run like this: "If there is a vector field on $M$ with such that ..., then $M$ is stochastically incomplete." The idea is to replace the Brownian trajectories by a deterministic vector field which should resemble in a large scale the velocity of the Brownian motion in the case of explosion.

9. Prove (or disprove) that the condition

$$
\int^{\infty} \frac{V(x, r)}{V^{\prime}(x, r)}=\infty
$$

for some $x \in M$, implies stochastic completeness of $M$. By Proposition 3.2, this is true for model manifolds.

The condition (18.9) is analogous to the condition (7.15) for parabolicity. The best known non-explosion test in terms of the volume function is given by Theorem 9.1 .

10. Let $M$ be a Cartan-Hadamard manifold and let $k(r)$ be a positive increasing function on $(0, \infty)$ such that the sectional curvature at any point $x \in M$ at any 2 -section is bounded above by $-C \rho^{2+\varepsilon}$ where $\rho=\rho(x)=\operatorname{dist}(x, o), o$ being a 
reference point, and $C>0$. Then, by Theorem 15.4(b), $M$ is not stochastically complete.

On the other hand, denote $\lambda_{e}(r):=\lambda_{1}(M \backslash \overline{B(o, r)})$. Since the curvature in $M \backslash \overline{B(o, r)}$ is bounded above by $-C r^{2+\varepsilon}$, the eigenvalue comparison results [137], [192] imply that

$$
\lambda_{e}(r) \geq C^{\prime} r^{2+\varepsilon}
$$

where $C^{\prime}=\frac{(d-1)^{2}}{4} C$. The question is whether (18.10) alone implies the explosion, without having to assume the curvature growth. In short, given a Cartan-Hadamard manifold $M$ satisfying (18.10), prove that $M$ is stochastically incomplete.

Presumably, one could show that the Green function on $M$ is summable (see Corollary 6.7). There is a heat kernel upper bound [80, Theorem 5.3] which takes into account the growth of $\lambda_{e}(r)$, but it is not good enough to show that $G$ is summable. Maybe one should assume also a matching upper bound of the volume $V(o, r)$.

\section{Liouville properties}

11. Give an efficient characterization of massive sets (see Section 4.4) in terms of their intrinsic geometry. For $D$-massive sets, such a characterization is given by Theorem 14.2. Since Theorem 13.10 provides a straightforward link of massive sets to bounded harmonic functions, there is a hope to obtain in this way a criterion for the $L^{\infty}$-Liouville property for harmonic functions.

12. Similarly, give characterizations of $\lambda$-massive sets (see Section 6) and $q$ massive sets (Section 13.2). The former may be used to produce a geometric criterion for stochastic completeness.

13. Suppose that $M$ has at most polynomial volume growth and bounded geometry. What is the necessary and sufficient geometric condition for the $L^{\infty}$-Liouville property for harmonic functions?

14. Generalize Theorem 13.10(c) to solutions to the Schrödinger equation (13.6). For example, prove or disprove that if $M$ contains $k$ disjoint $q$-massive sets (see Section 13.2), then the space of all bounded solutions to $\Delta u-q(x) u=0$ has the dimension at least $k$.

Similarly, can one state an analogue of Theorem 14.5 for the Schrödinger equation?

15. If manifolds $M$ and $N$ are stochastically complete, then the Riemannian product $M \times N$ is also stochastically complete (this follows from the fact that the heat kernel on $M \times N$ is the product of the heat kernels on $M$ and $N$ ). For parabolicity, this is not true: $\mathbb{R}^{1}$ and $\mathbb{R}^{2}$ are parabolic whereas their product $\mathbb{R}^{3}$ is not.

Suppose that $M$ admits the $q_{1}$-Liouville property (see Section 13.2) and $N$ admits the $q_{2}$-Liouville property. What $q$-Liouville property can be proved on $M \times N$ ?

It is natural to define the function $q=q(x, y)$ (where $x \in M, y \in N$ ) as follows: $q(x, y)=f\left(q_{1}(x), q_{2}(y)\right)$, where $f$ is a function in two variables. Recall that parabolicity is equivalent to the $q$-Liouville property with $q \in C_{0}^{\infty}$, whereas stochastic completeness is equivalent to that for $q \equiv 1$.

16. Prove (or disprove) that if manifolds $M$ and $N$ are parabolic, then the Riemannian product $M \times N$ possesses no bounded harmonic function except constant. This would explain (to some extent) why $\mathbb{R}^{d}$ admits the $L^{\infty}$-Liouville property for harmonic functions. 
It is not enough to assume that both $M$ and $N$ admit the $L^{\infty}$-Liouville property - see Section 13.5 for a counterexample.

17. Is the $q$-Liouville property (see Section 13.2) stable under quasi-isometry? If $q$ is compactly supported, then, by Theorem 5.1(7), the $q$-Liouville property is equivalent to parabolicity of $M$ and, therefore, is stable by Corollary 5.3. On the other hand, if $q \equiv 1$ then the $q$-Liouville property is equivalent to stochastic completeness that may be unstable.

Where is the borderline between these two possibilities? For example, let $q(x)=$ $\frac{1}{1+r^{2}}$ where $r:=\operatorname{dist}\left(x, x_{0}\right)$. Is it true that the $q$-Liouville property with this particular $q$ is stable under a quasi-isometry?

Note that the $q$-Liouville property is always preserved by a change of the metric and/or of $q$ within a compact set [88].

18. Theorem 8.2 ensures non-parabolicity provided a certain isoperimetric inequality holds on $M$. Is it possible to state a similar result for existence of a non-trivial bounded solution to the Schrödinger equation $\Delta u-q(x) u=0$ ?

19. Find a criterion for the $L^{\infty}$-Liouville property in the domain of revolution $\mathcal{D}_{f}$ given by (18.7). In other words, for what functions $f$ is any bounded harmonic function in $\mathcal{D}_{f}$ with the Neumann boundary condition on $\partial \mathcal{D}_{f}$ identically constant? For a model manifold $M_{\sigma}$, the $L^{\infty}$-Liouville property is equivalent to

$$
\int^{\infty} \frac{d r}{\sigma^{d-1}(r)} \int_{1}^{r} \sigma^{d-3}(\xi) d \xi=\infty
$$

(see [128] and [142]).

20. (M.Barlow) Let $M$ be non-parabolic. Prove (or disprove) that there exists an open subset $E \subset M$ with smooth boundary which admits a non-constant bounded harmonic function in $E$ with the Neumann boundary condition on $\partial E$. In other words, the $L^{\infty}$-Liouville property must fail in $\bar{E}$.

An intuition behind this conjecture is the following. Presumably, the set $E$ can be found so that it contains a "bottleneck" which separates $E$ into two subsets massive in $\bar{E}$. By Theorem 13.10(b), this will imply existence of a nontrivial bounded harmonic function on $\bar{E}$.

The assumption of non-parabolicity of $M$ is necessary since otherwise any subset $E$ is parabolic and so is the manifold $\bar{E}$, by Proposition 14.1. If $M=\mathbb{R}^{d}, d>2$, then $E$ can be constructed by gluing together two disjoint half-spaces via a compact tube. Another example of such a set $E$ being in addition diffeomorphic to a halfspace can be found in [73, Section 3].

Faber-Krahn inequality and capacity

21. Prove the following estimate of capacity

$$
\operatorname{cap}(B(x, r), B(x, R)) \geq \operatorname{const}\left(\int_{r}^{R} \frac{s d s}{V(x, s)}\right)^{-1}
$$

assuming the relative Faber-Krahn inequality (11.2). This will provide an alternative proof of Theorem 11.1.

22. Prove the following estimate of capacity (cf. (8.2))

$$
\operatorname{cap}(K, \Omega) \geq \operatorname{const}\left(\int_{|K|}^{|\Omega|} \frac{d v}{v^{2} \Lambda(v)}\right)^{-1}
$$


assuming the uniform Faber-Krahn inequality (10.5). This will provide an alternative proof of Theorem 10.2.

What is easy to prove is the following inequality:

$$
\operatorname{cap}(K, \Omega) \geq|K| \lambda_{1}(\Omega) \geq|K| \Lambda(|\Omega|),
$$

which follows from the definitions (4.12) of the capacity and (10.1) of $\lambda_{1}$, and which is clearly weaker than (18.11).

23. Given the Faber-Krahn inequality (10.5), prove an upper bound of the Green kernel

$$
G(x, y) \leq C \int_{V(y, r)}^{\infty} \frac{d v}{v^{2} \Lambda(v)},
$$

where $r=\operatorname{dist}(x, y)$. The motivation behind (18.13) is the following. The FaberKrahn inequality (10.5) follows from the isoperimetric inequality (8.1) if we set

$$
\Lambda(v):=\frac{1}{4}\left(\frac{f(v)}{v}\right)^{2}
$$

(see (10.9)). Assuming (8.1) and combining (4.24) with (8.2), we obtain

$$
\inf _{x \in \partial B(y, r)} G(x, y) \leq \int_{V(y, r)}^{\infty} \frac{d v}{f^{2}(v)}=\frac{1}{4} \int_{V(y, r)}^{\infty} \frac{d v}{v^{2} \Lambda(v)} .
$$

The point is, firstly, to obtain a pointwise upper bound for $G$ rather than an upper bound for inf $G$ as above; secondly, to assume a Faber-Krahn inequality rather than an isoperimetric inequality.

The Faber-Krahn inequality (10.5) implies an upper bound of the heat kernel [80, Theorem 5.1], which yields an upper estimate for $G$ upon integrating in time. However, this method does not give a very sharp result in the case when $G(x, y)$ decays superpolynomially as $r \rightarrow \infty$.

\section{Regular ends}

24. Assuming that $E$ is a regular end of $M$ (see Sections 14.3, 14.4), prove that the hypothesis

$$
\int^{\infty} \frac{s d s}{V_{E}(s)}<\infty
$$

implies non-parabolicity of $E$. Due to Theorem 14.6, it suffices to show that, for a regular end,

$$
\operatorname{cap}_{E}\left(B_{r}, B_{2 r}\right) \geq \mathrm{const} \frac{V_{E}(r)}{r^{2}},
$$

for all large $r$. By (18.12), this in turn would follow from $\lambda_{1}\left(B_{r}\right) \geq \frac{\text { const }}{r^{2}}$.

25. (P.Li [119]) Let $E$ be an end of a geodesically complete manifold $M$ and assume that the Ricci curvature is non-negative on $E$. Does this imply that the end $E$ is regular? (See Section 14.3 for the definitions.) The answer is "yes" if $E$ has a non-negative sectional curvature - see [121, Theorem 3.2]. Should the answer be in general "no", let us assume in addition that the volume comparison condition (VC) holds on $E$ (see Example 14.3 at the end of Section 14.4) and ask again whether $E$ is regular. More generally, do the conditions (P), (D) and (VC) (see Example 14.3) imply that $E$ is regular, without any curvature assumption? 


\section{Recurrence of the $\alpha$-process}

26. (I.McGillivray) Prove that if

$$
\int^{\infty} \frac{d t}{V\left(x, t^{1 / \alpha}\right)}=\infty
$$

for some $x \in M$, then the $\alpha$-process on $M$ is recurrent (see Section 16 for the definition of the $\alpha$-process). Theorem 16.2 guarantees the recurrence of the $\alpha$ process under the stronger assumption $V(x, r) \leq C r^{\alpha}$. Theorem 16.3 says that (18.14) is equivalent to the recurrence provided $M$ admits the relative Faber-Krahn inequality.

\section{Escape rate}

27. Produce a lower radius function $r(t)$ for the Brownian motion on manifolds with superpolynomial volume growth (see Section 17 for the definition of $r(t)$ ).

What is a lower radius $r(t)$ on a model manifold $M_{\sigma}$ (see Section 3.2) with $\sigma(r) \sim \exp \left(r^{\alpha}\right)$ ? The answer may be substantially different for small $\alpha>0$ and for $\alpha>2$. In the latter case, $M_{\sigma}$ is stochastically incomplete, by Proposition 3.2.

What is a lower radius on a covering manifold (see Section 11.2) given the volume growth function $V(r) \sim \exp \left(r^{\alpha}\right), 0<\alpha<1$ ? See [93] for the case of a polynomial volume growth.

Does there exist a manifold with a fast growing lower radius, say, $r(t)=t^{2}$ ?

28. Suppose that $r(t)$ is a lower radius for the Brownian motion on an arbitrary complete Riemannian manifold. Is it true that $2 r(t)$ is also a lower radius? For $\mathbb{R}^{d}$, this follows from the test (17.1) of Dvoretzky and Erdös (if $M$ is a manifold of non-negative Ricci curvature, then the sufficient condition (17.6) for a function $r(t)$ to be a lower radius is also stable under the change to $2 r(t))$. However, no direct way is known to see why $2 r(t)$ should be a lower radius in general.

Is there a manifold for which the following is true: if $R(t)$ is an upper radius, then $\frac{1}{2} R(t)$ is also an upper radius? In $\mathbb{R}^{d}$ this clearly fails.

\section{REFERENCES}

[1] Ahlfors L.V., Sur le type d'une surface de Riemann, C.R. Acad. Sci. Paris, 201 (1935) 30-32.

[2] Ahlfors L.V., On the characterization of hyperbolic Riemann surfaces, Ann. Acad. Sci. Fenn. Series A I. Math., 125 (1952) MR 14:970a

[3] Ahlfors L.V., Sario L., Riemann surfaces, Princeton mathematical series 26, Princeton Univ. Press, 1960. MR 22:5729

[4] Albeverio S., Kolokoltsov V.N., The rate of escape for some Gaussian processes and the scattering theory for their small perturbations, Stochastic Process. Appl. 67 (1997) 139-159. MR 98g:60076

[5] Ancona A., Théorie du potentiel sur des graphes et des variétés, in: Cours de l'Ecole d'été de probabilités de Saint-Flour, 1988, Lecture Notes in Math. 1427, Springer, (1990) 4-112. MR 92g:31012

[6] Azencott R., Behavior of diffusion semi-groups at infinity, Bull. Soc. Math. (France), 102 (1974) 193-240. MR 50:8725

[7] Bader R., Parreau M., Domaines non-compacts et classification des surfaces de Riemann, C.R. Acad. Sci. Paris, 232 (1951) MR 12:603a

[8] Barlow M., Perkins A., Symmetric Markov chains in $\mathbb{Z}^{d}$ : how fast can they move?, Probab. Th. Rel. Fields, 82 (1989) 95-108. MR 90j:60067

[9] Bass H., The degree of polynomial growth of finitely generated groups, Proc. London Math. Soc., 25 (1972) 603-614. MR 52:577

[10] Batty C.J.K., Asymptotic stability of Schrödinger semigroups: path integral methods, Math. Ann., 292 (1992) no.3, 457-492. MR 93g:47050 
[11] Benjamini I., Cao J., Examples of simply-connected Liouville manifolds with positive spectrum, Differential Geom. Appl. 6 (1996) 31-50. MR 97b:53036

[12] Bernstein S., Sur un théorème de géométrie et ses applications aux équations aux dérivées partielles du type elliptique, Comm. Inst. Sci. Math. Mech. Univ. Kharkov, 15 (1915-17) 38-45.

[13] Bers L., Non-linear elliptic equations without non-linear entire solutions, J. Rat. Mech. Anal., 3 (1954) 767-787. MR 16:707b

[14] Bingham N.H., Variants on the law of the iterated logarithm, Bull. London Math. Soc., 18 (1986) 433-467. MR 87k:60087

[15] Bishop R., O'Neill B., Manifolds of negative curvature, Trans. Amer. Math. Soc., 145 (1969) 1-49. MR 40:4891

[16] Bliedtner J., Hansen W., Potential theory - an analytic and probabilistic approach to balayage, Universitext, Springer, Berlin-Heidelberg-New York-Tokyo, 1986. MR 88b:31002

[17] Bombieri E., Giusti E., Harnack's inequality for elliptic differential equations on minimal surfaces, Invent. Math., 15 (1972) 24-46. MR 46:8057

[18] Brooks R., A relation between growth and the spectrum of the Laplacian, Math. Z., 178 (1981) 501-508. MR 83a:58089

[19] Carlen E.A., Kusuoka S., Stroock D.W., Upper bounds for symmetric Markov transition functions, Ann. Inst. H. Poincaré, Proba. et Stat., (1987) suppl. au no.2, 245-287. MR 88i:35066

[20] Carron G., Inégalités isopérimétriques de Faber-Krahn et conséquences, in: Actes de la table ronde de géométrie différentielle en l'honneur de Marcel Berger, Collection SMF Séminaires et congrès, no.1, 1994. MR 97m:58198

[21] Chavel I., Eigenvalues in Riemannian geometry, Academic Press, New York, 1984. MR 86g:58140

[22] Chavel I., Riemannian geometry : a modern introduction, Cambridge Tracts in Mathematics 108, Cambridge University Press, 1993. MR 95j:53001

[23] Chavel I., Feldman E.A., Isoperimetric constants, the geometry of ends, and large time heat diffusion in Riemannian manifolds, Proc. London Math. Soc., 62 (1991) 427-448. MR 93c: 58209

[24] Chavel I., Feldman E.A., Modified isoperimetric constants, and large time heat diffusion in Riemannian manifolds, Duke Math. J., 64 (1991) no.3, 473-499. MR 92m:58132

[25] Chavel I., Karp L., Large time behavior of the heat kernel: the parabolic $\lambda$-potential alernative, Comment. Math. Helvetici, 66 (1991) 541-556. MR 93a:58160

[26] Cheeger J., A lower bound for the smallest eigenvalue of the Laplacian, in: Problems in Analysis: A Symposium in honor of Salomon Bochner, Princeton University Press. Princeton, 1970. 195-199. MR 53:6645

[27] Cheeger J., Yau S.-T., A lower bound for the heat kernel, Comm. Pure Appl. Math., 34 (1981) 465-480. MR 82i:58065

[28] Cheng S.Y., Yau S.-T., Differential equations on Riemannian manifolds and their geometric applications, Comm. Pure Appl. Math., 28 (1975) 333-354. MR 52:6608

[29] Choquet G., Theory of capacities, Ann. Inst. Fourier, 155 no.5, 131-395. MR 18:295g

[30] Chung K.L., Zhao Z., From Brownian motion to Schrödinger's equation, A series of Comprehensive Studies in Mathematics 312, Springer, 1985. MR 96f:60140

[31] Chung L.O., Existence of harmonic $L^{1}$ functions in complete Riemannian manifolds, Proc. Amer. Math. Soc. 88 (1983) 531-532. MR 84k:31006

[32] Constantinescu C., Cornea A., Potential theory on harmonic spaces, Springer-Verlag, 1972. MR 54:7817

[33] Coulhon T., Noyau de la chaleur et discrétisation d'une variété riemanniene, Israel J. Math., 80 (1992) 289-300. MR 93k:58213

[34] Coulhon T., Ultracontractivity and Nash type inequalities, J. Funct. Anal., 141 (1996) 510-539. MR 97j: 47055

[35] Coulhon T., Grigor'yan A., Heat kernel, volume growth and anti-isoperimetric inequalities, C.R. Acad. Sci. Paris, Sér. I Math., 322 (1996) 1027-1032. MR 97f:58125

[36] Coulhon T., Grigor'yan A., On-diagonal lower bounds for heat kernels on non-compact manifolds and Markov chains, Duke Math. J., 89 no.1, (1997) 133-199. MR 98e:58159

[37] Coulhon T., Grigor'yan A., Random walks on graphs with regular volume growth, Geom. and Funct. Analysis, 8 (1998) 656-701. CMP 98:16 
[38] Coulhon T., Saloff-Coste L., Isopérimétrie pour les groupes et les variétés, Revista Mathemática Iberoamericana, 9 (1993) no.2, 293-314. MR 94g:58263

[39] Coulhon T., Saloff-Coste L., Harnack inequality and hyperbolicity for p-Laplacian with applications to Picard type theorems, preprint.

[40] Cranston M., A probabilistic approach to Martin boundaries for manifolds with end, Prob. Theory and Related Fields, 96 (1993) 319-334. MR 95d:58141

[41] Davies E.B., $L^{1}$ properties of second order elliptic operators, Bull. London Math. Soc., 17 (1985) no.5, 417-436. MR 87g:58126

[42] Davies E.B., Gaussian upper bounds for the heat kernel of some second-order operators on Riemannian manifolds, J. Funct. Anal., 80 (1988) 16-32. MR 90k:58213

[43] Davies E.B., Heat kernels and spectral theory, Cambridge University Press, Cambridge, 1989. MR 90e:35123

[44] Davies E.B., Heat kernel bounds, conservation of probability and the Feller property, J. d'Analyse Math., 58 (1992) 99-119. MR 94e:58136

[45] Davies E.B., Non-Gaussian aspects of heat kernel behaviour, J. London Math. Soc., 55 (1997) no.1, 105-125. MR 97i:58169

[46] Davies E.B., Pang M.M.H., Sharp heat kernel bounds for some Laplace operators, Quart. J. Math., 40 (1989) 281-290. MR 91i:58142

[47] Deny J., Méthodes Hilbertiennes et théorie du potentiel, Potential Theory, Centro Internazionale Matematico Estivo, Edizioni Cremonese, Roma, 1970. MR 44:1833

[48] Dodziuk J., Maximum principle for parabolic inequalities and the heat flow on open manifolds, Indiana Univ. Math. J., 32 (1983) no.5, 703-716. MR 85e:58140

[49] Dodziuk J., Difference equations, isoperimetric inequalities and transience of certain random walks, Trans. Amer. Math. Soc., 284 (1984) 787-794. MR 85m:58185

[50] Donnelly H., Bounded harmonic functions and positive Ricci curvature, Math. Z., 191 (1986) 559-565. MR 87e:58204

[51] Doob J., Classical potential theory and its probabilistic counterpart, Springer, 1983. MR 85k:31001

[52] Doyle P.G., On deciding whether a surface is parabolic or hyperbolic, Contemporary Mathematics, 73 (1988) 41-49. MR 89h:53043

[53] Doyle P.G., Snell J.L., Random walks and electric networks, Carus Mathematical Monographs 22, Mathematical Association of America, Washington, DC, 1984. MR 89a:94023

[54] Dvoretzky A., Erdös P., Some problems on random walk in space, Proc. Second Berkeley Symposium on Math. Stat. and Probability, University of California Press, 1951. 353-368. MR 13:852b

[55] Dynkin E.B., Markov processes, Springer, 1965. MR 33:1887

[56] Einstein A., On the motion of small particles suspended in liquids at rest required by the molecular-kinetic theory of heat, Annalen der Physik, 1905. See also Einstein's miraculous year, ed. John Stachel, Princeton University Press, 1998. 71-85.

[57] Elworthy K.D., Stochastic differential equations on manifolds, LMS Lecture Notes Series 70, Cambridge - New York, 1982. MR 84d:58080

[58] Feller W., The parabolic differential equations and the associated semigroup of transformations, Ann. Math., 55 no.3, (1952) 467. MR 13:948a

[59] Feller W., Diffusion processes in one dimension, Trans. Amer. Math. Soc., 77 no.1, (1954) 1-31. MR 16:150d

[60] Feller W., An introduction to probability theory and its applications, Vol.1,2, New York: Wiley, 1966. MR 37:3604; MR 35:1048

[61] Fernández, J.L., On the existence of Green's function on Riemannian manifolds, Proc. AMS, 96 (1986) 284-286. MR 87b:31005

[62] Finn R., On problem of type, with application to elliptic partial differential equations, $J$. Rat. Mech. Anal., 3 (1954) 789-799. MR 16:708a

[63] Fukushima M., Dirichlet forms and Markov processes, North Holland, Kodansha, 1980. MR 81f: 60105

[64] Fukushima M., Oshima Y., Takeda M., Dirichlet forms and symmetric Markov processes, Studies in Mathematics 19, De Gruyter, 1994. MR 96f:60126

[65] Gaffney M. P., A special Stokes theorem for complete Riemannian manifolds, Annals of Math., 60 (1945) 140-145. MR 16:358a 
[66] Gaffney M. P., The conservation property of the heat equation on Riemannian manifolds, Comm. Pure Appl. Math., 12 (1959) 1-11. MR 21:892

[67] Gerl P., Random walks on graphs, Lecture Notes Math 1210, Springer, 1986. 285-303. MR 88m:60178

[68] Gilbarg D., Trudinger N., Elliptic partial differential equations of second order, Springer, 1977. MR 57:13109

[69] Greene R., Wu W., Function theory of manifolds which possess a pole, Lecture Notes Math 699, Springer, 1979. MR 81a:53002

[70] Grigor'yan A., On the existence of a Green function on a manifold, (in Russian) Uspekhi Matem. Nauk, 38 (1983) no.1, 161-162. Engl. transl. Russian Math. Surveys, 38 (1983) no.1, 190-191. MR 84i:58128

[71] Grigor'yan A., On the existence of positive fundamental solution of the Laplace equation on Riemannian manifolds, (in Russian) Matem. Sbornik, 128 (1985) no.3, 354-363. Engl. transl. Math. USSR Sb., 56 (1987) 349-358. MR 87d:58140

[72] Grigor'yan A., On stochastically complete manifolds, (in Russian) DAN SSSR, 290 (1986) no.3, 534-537. Engl. transl. Soviet Math. Dokl., 34 (1987) no.2, 310-313. MR 88a:58209

[73] Grigor'yan A., On Liouville theorems for harmonic functions with finite Dirichlet integral, (in Russian) Matem. Sbornik, 132 (1987) no.4, 496-516. Engl. transl. Math. USSR Sbornik, 60 (1988) no.2, 485-504. MR 88g:35068

[74] Grigor'yan A., Set of positive solutions of Laplace-Beltrami equation on special type of Riemannian manifolds, (in Russian) Izv. Vyssh. Uchebn. Zaved., Matematika, (1987) no.2, 30-37. Engl. transl. Soviet Math (Iz.VUZ), 31 (1987) no.2, 48-60. MR 88g:58187

[75] Grigor'yan A., Stochastically complete manifolds and summable harmonic functions, (in Russian) Izv. AN SSSR, ser. matem., 52 no.5, (1988) 1102-1108. Engl. transl. Math. USSR Izvestiya, 33 no.2, (1989) 425-432. MR 90a:58189

[76] Grigor'yan A., Bounded solutions of the Schrödinger equation on non-compact Riemannian manifolds, (in Russian) Trudy seminara I.G.Petrovskogo, (1989) no.14, 66-77. Engl. transl. Journal of Soviet Math., 51 (1990) no.3, 2340-2349. MR 90m:35050

[77] Grigor'yan A., Dimension of spaces of harmonic functions, (in Russian) Mat. Zametki, 48 (1990) no.5, 55-61. Engl. transl. Math. Notes, 48 (1990) no.5, 1114-1118. MR 92j:31015

[78] Grigor'yan A., The heat equation on non-compact Riemannian manifolds, (in Russian) Matem. Sbornik, 182 (1991) no.1, 55-87. Engl. transl. Math. USSR Sb., 72 (1992) no.1, 47-77. MR 92h:58189

[79] Grigor'yan A., Heat kernel on a non-compact Riemannian manifold, in: 1993 Summer Research Institute on Stochastic Analysis, ed. M.Pinsky et al., Proceedings of Symposia in Pure Mathematics, 57 (1994) 239-263. MR 96f:58155

[80] Grigor'yan A., Heat kernel upper bounds on a complete non-compact manifold, Revista Mathemática Iberoamericana, 10 (1994) no.2, 395-452. MR 96b:58107

[81] Grigor'yan A., Integral maximum principle and its applications, Proc. Roy. Soc. Edinburgh, 124A (1994) 353-362. MR 95c:35045

[82] Grigor'yan A., Heat kernel on a manifold with a local Harnack inequality, Comm. Anal. Geom., 2 (1994) no.1, 111-138. MR 95m:58117

[83] Grigor'yan A., Escape rate of Brownian motion on weighted manifolds, to appear in Applicable Analysis.

[84] Grigor'yan A., On non-parabolicity of Riemannian manifolds, preprint.

[85] Grigor'yan A., Hansen W., A Liouville property for Schrödinger operators, Math. Ann., 312 (1998) 659-716.

[86] Grigor'yan A., Kelbert M., Range of fluctuation of Brownian motion on a complete Riemannian manifold, Ann. Prob., 26 (1998) 78-111. CMP 98:11

[87] Grigor'yan A., Kelbert M., On Hardy-Littlewood inequality for Brownian motion on Riemannian manifolds, to appear in J. London Math. Soc.

[88] Grigor'yan A., Nadirashvili N.S., Liouville theorems and exterior boundary value problems, (in Russian) Izv. Vyssh. Uchebn. Zaved., Matematika, (1987) no.5, 25-33. Engl. transl. Soviet Math. (Iz.VUZ), 31 (1987) no.5, 31-42. MR 88k:35050

[89] Gromov M., Groups of polynomial growth and expanding maps, Publ. Math. I.H.E.S., 53 (1981) 53-73. MR 83b:53041; Appendix MR 83b:53042

[90] Guivarc'h Y., Sur la loi des grands nombres et le rayon spectral d'une marche aléatoire, Journée sur les marches aléatoires, Astérisque, 74 (1980) 47-98. MR 82g:60016 
[91] Gushchin A.K., On the uniform stabilization of solutions of the second mixed problem for a parabolic equation, (in Russian) Matem. Sbornik, 119(161) (1982) no.4, 451-508. Engl. transl. Math. USSR Sb., 47 (1984) 439-498. MR 84g:35021b

[92] Hebey E., Sobolev spaces on Riemannian manifolds, Lecture Notes in Math. 1635, Springer, (1996) MR 98k:46049

[93] Hebisch W., Saloff-Coste, L., Gaussian estimates for Markov chains and random walks on groups, Ann. Prob., 21 (1993) 673-709. MR 94m:60144

[94] Holopainen I., Rough isometries and p-harmonic functions with finite Dirichlet integral, Revista Mathemática Iberoamericana, 10 (1994) 143-176. MR 95d:31006

[95] Holopainen I., Solutions of elliptic equations on manifolds with roughly Euclidean ends, Math. Z., 217 (1994) 459-477. MR 95j:58179

[96] Holopainen I., Volume growth, Green's functions and parabolicity of ends, to appear in Duke Math. J.

[97] Hsu E.P., Heat semigroup on a complete Riemannian manifold, Ann. Probab., 17 (1989) 1248-1254. MR 90j:58158

[98] Hunt G.A., On positive Green's functions, Proc. Nat. Acad. Sci. USA, 40 (1954) 816-818. MR 16:135b

[99] Ichihara K., Curvature, geodesics and the Brownian motion on a Riemannian manifold. I Recurrence properties, Nagoya Math. J., 87 (1982) 101-114. MR 84m:58166a

[100] Ichihara K., Curvature, geodesics and the Brownian motion on a Riemannian manifold. II Explosion properties, Nagoya Math. J., 87 (1982) 115-125. MR 84m:58166b

[101] Ishige K., Murata M., Parabolic equations whose nonnegative solutions are determined only by their initial values, preprint.

[102] Itô K., On stochastic differential equations on differentiable manifolds, 1, Nagoya Math. J., 1 (1950) 35-47. MR 12:425g

[103] Itô K., McKean H., Diffusion processes and their sample paths, Springer, Berlin, 1965. MR 33:8031

[104] Kaimanovich V.A., Vershik A.M., Random walks on discrete groups: boundary and entropy, Ann. Prob., 11 (1983) 453-490. MR 85d:60024

[105] Kakutani S., Random walk and the type problem of Riemann surfaces, Princeton Univ. Press, (1961) 95-101. MR 15:25e

[106] Kanai M., Rough isometries, and combinatorial approximations of geometries of noncompact Riemannian manifolds, J. Math. Soc. Japan, 37 (1985) 391-413. MR 87d:53082

[107] Kanai M., Rough isometries and the parabolicity of manifolds, J. Math. Soc. Japan, 38 (1986) 227-238. MR 87e:53066

[108] Karp L., Subharmonic functions, harmonic mappings and isometric immersions, in: Seminar on Differential Geometry, ed. S.T.Yau, Ann. Math. Stud. 102, Princeton, 1982.

[109] Karp L., Li P., The heat equation on complete Riemannian manifolds, unpublished.

[110] Keselman, V.M., Riemannian manifolds of $\alpha$-parabolic types, (in Russian) Izv. Vysshikh Uchebn. Zaved. Matematika, (1985) no.4, 81-83. MR 86m:31009

[111] Khas'minskii R.Z., Ergodic properties of recurrent diffusion prossesses and stabilization of solution to the Cauchy problem for parabolic equations, Theor. Prob. Appl., 5 no.2, (1960) 179-195. MR 24:A3695

[112] Kobayashi S., Nomizu K., Foundations of differential geometry, Interscience Publishers, New York, Vol I:1963. Vol II:1969. MR 97c:53001a

[113] Kondrat'ev V.A., Landis E.M., Qualitative theory of linear second-order partial differential equations, (in Russian) Itogi Nauki i Techniki, serija Sovremennye Problemy Matematiki, Fundamental'nye Napravlenija 32, VINITI, Moscow, 1988. 99-215. Engl. transl. in: Partial Differential Equations III, Encyclopedia of Math. Sci. 32, Springer Verlag, 1990. MR 92m:35015

[114] Koranyi A., Taylor J.C., Minimal solutions of the heat equation and uniqueness of the positive Cauchy problem on homogeneous spaces, Proc. Amer. Math. Soc., 94 (1985) 273-278. MR 86i:58126

[115] Kuzmenko Yu.T., Molchanov S.A., Counterexamples to Liouville-type theorems, (in Russian) Vestnik Moskov. Univ. Ser. I Mat. Mekh., (1979) no.6, 39-43. Engl. transl. Moscow Univ. Math. Bull., 34 (1979) 35-39. MR 81c:58063 
[116] Landis E.M., The second order equations of elliptic and parabolic type, (in Russian) Nauka, Moscow, 1971. Engl. transl. Transl. of Mathematical Monographs 171, AMS publications, 1998. MR 98k:35034

[117] Li P., Uniqueness of $L^{1}$-solutions for the Laplace equation and the heat equation on Riemannian manifolds, J. Diff. Geom., 20 (1984) no.2, 447-457. MR 86h:58133

[118] Li P., Large time behavior of the heat equation on complete manifolds with nonnegative Ricci curvature, Ann. Math., 124 (1986) 1-21. MR 87k:58259

[119] Li P., Curvature and function theory on Riemannian manifolds, preprint.

[120] Li P., Schoen R., $L^{p}$ and mean value properties of subharmonic functions on Riemannian manifolds, Acta Math., 153 (1984) 279-301. MR 86j:58147

[121] Li P., Tam L.F., Positive harmonic functions on complete manifolds with non-negative curvature outside a compact set, Ann. Math., 125 (1987) 171-207. MR 88m:58039

[122] Li P., Tam L.F., Symmetric Green's functions on complete manifolds, Amer. J. Math., 109 (1987) 1129-1154. MR 89f:58129

[123] Li P., Tam L.F., Harmonic functions and the structure of complete manifolds, J. Diff. Geom., 35 (1992) 359-383. MR 93b:53033

[124] Li P., Tam L.F., Green's function, harmonic functions and volume comparison, J. Diff. Geom., 41 (1995) 277-318. MR 96f:53054

[125] Li P., Wang J., Convex hull properties of harmonic maps, J. Diff. Geom., 48 (1998) 497-530. CMP 98:16

[126] Li P., Yau S.-T., On the parabolic kernel of the Schrödinger operator, Acta Math., 156 (1986) no.3-4, 153-201. MR 87f:58156

[127] Littman W., Stampaccia G., Weinberger H.F., Regular points for elliptic equations with discontinuous coefficients, Ann. Scuola Norm. Sup. Pisa (3), 17 (1963) 43-77. MR 28: 4228

[128] Losev A.G., Some Liouville theorems on Riemannian manifolds of a special type, Izv. Vyssh. Uchebn. Zaved. Matematika, (1991) no.12, 15-24. MR 94c:31010

[129] Lyons T., Instability of the Liouville property for quasi-isometric Riemannian manifolds and reversible Markov chains, J. Diff. Geom., 26 (1987) 33-66. MR 88k:31012

[130] Lyons T., Random thoughts on reversible potential theory, in: Summer School in Potential Theory, Joensuu 1990, ed. Ilpo Laine, Publications in Sciences 26, University of Joensuu, 71-114. MR 93k:31008

[131] Lyons T., Instability of the conservative property under quasi-isometries, J. Diff. Geom., 34 (1991) 483-489. MR 92k:58275

[132] Lyons T., Sullivan D., Function theory, random paths and covering spaces, J. Diff. Geom., 19 (1984) 299-323. MR 86b:58130

[133] Malliavin P., Stochastic Analysis, Springer, 1997. MR 99b:60073

[134] Maz'ya V.G., Sobolev spaces, (in Russian) Izdat. Leningrad Gos. Univ. Leningrad, 1985. Engl. transl. Springer-Verlag, 1985. MR 87g:46056

[135] McGillivray I., A recurrence condition for some subordinated strongly local Dirichlet forms, Forum Math., 9 (1997) 229-246. MR 97m:60111

[136] McKean H.P., Stochastic integrals, Academic Press, 1969. MR 40:947

[137] McKean H.P., An upper bound to the spectrum of $\Delta$ on a manifold of negative curvature, J. Diff. Geom., 4 (1970) 359-366. MR 42:1009

[138] Mikljukov V.M., A new approach to the Bernstein theorem and to related questions of equations of minimal surface type, (in Russian) Matem. Sbornik, 108 (1979) no.2, 268289. Engl. transl. Math. USSR Sbornik, 36 (1980) no.2, 251-271. MR 80e:53005

[139] Milnor J., On deciding whether a surface is parabolic or hyperbolic, Amer. Math. Monthly, 84 (1977) 43-46. MR 55:1257

[140] Molchanov S.A., Diffusion processes and Riemannian geometry, (in Russian) Uspekhi Matem. Nauk, 30 (1975) no.1, 3-59. Engl. transl. Russian Math. Surveys, 30 (1975) no.1, 1-63. MR 54:1404

[141] Mori A., On the existence of harmonic functions on a Riemann surface, J. Fac. Sci. Tokyo Univ., Section I., 6 (1951) MR 13:735g

[142] Murata M., Positive harmonic functions on rotationary symmetric Riemannian manifolds, in: Potential Theory, ed. M.Kishi, Walter de Gruyter, Berlin, 1992. 251-259. MR 93g:58158 
[143] Murata M., Uniqueness and nonuniqueness of the positive Cauchy problem for the heat equation on Riemannian manifolds, Proceedings of AMS, 123 no.6, (1995) 1923-1932. MR 95g:58231

[144] Myrberg P.J., Über die Existenz der Greenschen Funktionen auf einer gegebenen Riemannschen Fläche, Acta Math., 61 (1933)

[145] Nadirashvili N.S., A theorem of Liouville type on a Riemannian manifold, (in Russian) Uspekhi Matem. Nauk, 40 (1985) no.5, 259-260. Engl. transl. Russian Math. Surveys, 40 (1986) no.5, 235-236. MR 87d:31011

[146] Nakai, M., On Evans potential, Proc. Japan. Acad., 38 (1962) 624-629. MR 27:297

[147] Nash J., Continuity of solutions of parabolic and elliptic equations, Amer. J. Math., 80 (1958) 931-954. MR 20:6592

[148] Nevanlinna R., Über die Lösbarkeit des Dirichletschen Problems für eine Riemannsche Fläche, Göttinger Nachr., 1 no.14, (1939)

[149] Nevanlinna R., Ein Satz über offenen Riemannsche Flächen, Ann. Acad. Sci. Fenn. Part A., 54 (1940) 1-18. MR 2:276c

[150] Nevanlinna R., Quadratische integrierbare Differentiale auf einer Riemannschen Mannigfaltigkeit, Ann. Acad. Sci. Fenn. Series A I. Math., 1 (1941). MR 7:427e

[151] Nevanlinna R., Über die Existenz von beschränkten Potentialfunktionen auf Flächen von unendlichen Geschlecht, Math. Zeitschrift, 52 (1950) 599-604. MR 12:493a

[152] Ohtsuka M., Dirichlet problems on Riemann surfaces and conformal mappings, Nagoya Math. J., 3 (1951) MR 13:642f

[153] Ôkura H., Capacitary inequalities and global properties of symmetric Dirichlet forms, Dirichlet Forms and Stochastic Processes, 1995, 291-303. MR 97e:60128

[154] Oleinik O.A., Radkevich E.V., The method of introducing a parameter in the study of evolutionary equations, (in Russian) Uspekhi Matem. Nauk, 33 no.5, (1978) 7-76. Engl. transl. Russian Math. Surveys, 33 no.5, (1978) 7-84. MR 80d:35074

[155] Osserman R., Remarks on minimal surfaces, Comm. Pure Applied Math., 12 (1959) no.2, 233-239. MR 21:6590

[156] Osserman R., Hyperbolic surfaces of the form $z=f(x, y)$, Math. Ann., 144 (1961) 77-79. MR 31:337

[157] Osserman R., A survey of minimal surfaces, Dover, New York, 1986. MR 87j:53012

[158] Petersen P., Riemannian geometry, Graduate Texts in Mathematics 171, Springer, 1998. MR 98m:53001

[159] Pólya G., Über eine Aufgabe der Wahrscheinlichkeitstheorie betreffend die Irrfahrt im Straßennetz, Math. Ann., 84 (1921) 149-160.

[160] Pinchover Y., On non-existence of any $\lambda_{0}$-invariant positive harmonic function, a counter example to Stroock's conjecture, Comm. Partial Differential Equations 20 (1995) 1831-1846. MR 96e: 60145

[161] Robinson D.W., Elliptic operators and Lie groups, Oxford Math. Mono., Clarendon Press, Oxford-New York-Tokyo, 1991. MR 92m:58133

[162] Rosenberg S., The Laplacian on a Riemannian manifold, Student Texts 31, London Mathematical Society, 1991. MR 98k:58206

[163] Royden H.L., Harmonic functions on open Riemann surfaces, Trans. Amer. Math. Soc., 73 (1952) 40-94. MR 14:167d

[164] Saloff-Coste L., A note on Poincaré, Sobolev, and Harnack inequalities, Duke Math J., I.M.R.N., 2 (1992) 27-38. MR 93d:58158

[165] Saloff-Coste L., Parabolic Harnack inequality for divergence form second order differential operators, Potential Analysis, 4 (1995) 429-467. MR 96m:35031

[166] Saloff-Coste L., Lectures on finite Markov chains, Lecture Notes in Math. 1665, 1997. MR 99b:60119

[167] Sario L., Nakai M., Wang C., Chung L.O., Classification theory of Riemannian manifolds, Lecture Notes in Math., 605 1977. MR 58:22612

[168] Schoen R., Simon L., Regularity of simply connected surfaces with quasiconformal Gauss map, in: Seminar on minimal submanifolds, ed. E.Bombieri, Ann. of Math. Studies 103, Princeton, 1983. 127-145. MR 87b:53009

[169] Schoen R., Yau S.-T., Lectures on Differential Geometry, Conference Proceedings and Lecture Notes in Geometry and Topology 1, International Press, 1994. MR 97d:53001 
[170] Schoen R., Yau S.-T., Lectures on Harmonic maps, Conference Proceedings and Lecture Notes in Geometry and Topology 2, International Press, 1997. MR 98i:58072

[171] Strichartz R.S., Analysis of the Laplacian on the complete Riemannian manifold, J. Funct. Anal., 52 (1983) no.1, 48-79. MR 84m:58138

[172] Stroock D.W., Probability Theory. An analytic view, Cambridge Univ. Press, 1993. MR 95f: 60003

[173] Sturm K-Th., Analysis on local Dirichlet spaces I. Recurrence, conservativeness and $L^{p}$ Liouville properties, J. Reine. Angew. Math., 456 (1994) 173-196. MR 95i:31003

[174] Sturm K-Th., Sharp estimates for capacities and applications to symmetrical diffusions, Probability theory and related fields, 103 (1995) no.1, 73-89. MR 97a:31004

[175] Sung C.-J., Tam L.-F., Wang J., Spaces of harmonic functions, preprint.

[176] Täcklind S., Sur les classes quasianalytiques des solutions des équations aux dérivées partielles du type parabolique, Nova Acta Regalis Societatis Scientiarum Uppsaliensis, (4), 10 no.3, (1936) 3-55.

[177] Takeda M., On a martingale method for symmetric diffusion process and its applications, Osaka J. Math, 26 (1989) 605-623. MR 91d:60193

[178] Tichonov A.N., Uniqueness theorems for the equation of heat conduction, (in Russian) Matem. Sbornik, 42 (1935) 199-215.

[179] Tsuji M., Potential theory in modern function theory, Tokyo: Maruzen, 1959. MR 22:5712

[180] Urakawa H., Geometry of Laplace-Beltrami operator on a complete Riemannian manifold, in: Progress in differential geometry, Advanced Studies in Pure Math. 22, Math. Soc. Japan, Tokyo, (1993) 347-406. MR 95b:58160

[181] Varopoulos N.Th., The Poisson kernel on positively curved manifolds, J. Funct. Anal., 44 (1981) 359-380. MR 84h:58142a

[182] Varopoulos N.Th., Green's function on positively curved manifolds II, J. Funct. Anal., 45 (1982) no.2, 170-176. MR 84h:58142b

[183] Varopoulos N.Th., Random walks on soluble groups, Bull. Sc. Math., 22ème série, 107 (1983) 337-344. MR 85e:60076

[184] Varopoulos N.Th., Potential theory and diffusion of Riemannian manifolds, in: Conference on Harmonic Analysis in honor of Antoni Zygmund. Vol I, II, Wadsworth Math. Ser., Wadsworth, Belmont, Calif., 1983. 821-837. MR 85a:58103

[185] Varopoulos N.Th., Brownian motion and random walks on manifolds, Ann. Inst. Fourier, 34 (1984) 243-269. MR 85m:58186

[186] Varopoulos N.Th., Hardy-Littlewood theory for semigroups, J. Funct. Anal., 63 (1985) no.2, 240-260. MR 87a:31011

[187] Varopoulos N.Th., Théorie du potentiel sur des groupes et des variétés, C. R. Acad. Sci. Paris Sér. I Math., 302 (1986) 203-205. MR 87c:22020

[188] Varopoulos N.Th., Saloff-Coste L., Coulhon T., Analysis and geometry on groups, Cambridge University Press, Cambridge, 1992. MR 95f:43008

[189] Wiener N., Differential space, J. Math. Phys. Mass. Techn., 2 (1923) 131-174.

[190] Woess W., Random walks on infinite graphs and groups - a survey on selected topics, Bull. $L M S, 26$ (1994) 1-60. MR 94i:60081

[191] Woess W., Random walks on infinite graphs and groups, in preparation.

[192] Yau S.-T., Isoperimetric constant and the first eigenvalue of a compact Riemannian manifold, Ann. Sci. Ecole Norm. Sup., 4th serie, 8 no.4, (1975) 487-507. MR 53:1478

[193] Yau S.-T., Some function-theoretic properties of complete Riemannian manifolds and their applications to geometry, Indiana Math. J., 25 (1976) 659-670. MR 54:5502

[194] Yau S.-T., On the heat kernel of a complete Riemannian manifold, J. Math. Pures Appl., ser. 9, 57 (1978) 191-201. MR 81b:58041

[195] Yosida K., Functional analysis, Springer, 1980. MR 82i:46002

Department of Mathematics, Imperial College, London SW7 2BZ, United Kingdom

E-mail address: a.grigoryan@ic.ac.uk

$U R L:$ http://www.ma.ic.ac.uk/ grigor 